\title{
Analytical and Computational Studies of Gaseous and Dusty Combustion: From the Impacts of Local Flame Speed Variations to a Global Predictive Mining Fire Scenario
}

Sinan Demir

Follow this and additional works at: https://researchrepository.wvu.edu/etd

\author{
Recommended Citation \\ Demir, Sinan, "Analytical and Computational Studies of Gaseous and Dusty Combustion: From the \\ Impacts of Local Flame Speed Variations to a Global Predictive Mining Fire Scenario" (2017). Graduate \\ Theses, Dissertations, and Problem Reports. 5471. \\ https://researchrepository.wvu.edu/etd/5471
}

This Dissertation is protected by copyright and/or related rights. It has been brought to you by the The Research Repository @ WVU with permission from the rights-holder(s). You are free to use this Dissertation in any way that is permitted by the copyright and related rights legislation that applies to your use. For other uses you must obtain permission from the rights-holder(s) directly, unless additional rights are indicated by a Creative Commons license in the record and/ or on the work itself. This Dissertation has been accepted for inclusion in WVU Graduate Theses, Dissertations, and Problem Reports collection by an authorized administrator of The Research Repository @ WVU.

For more information, please contact researchrepository@mail.wvu.edu. 


\title{
Analytical and Computational Studies of Gaseous and Dusty Combustion: From the Impacts of Local Flame Speed Variations to a Global Predictive Mining Fire Scenario
}

\section{Sinan Demir}

Dissertation submitted

to the Statler College of Engineering and Mineral Resources at West Virginia University

in partial fulfillment of the requirements for the degree of

Doctor of Philosophy in

Mechanical Engineering

V'yacheslav Akkerman, Ph.D., Chair

Richard Bajura, Ph.D.

John M. Kuhlman, Ph.D.

Ali S. Rangwala, Ph.D.

Cosmin Dumitrescu, Ph.D.

Department of Mechanical and Aerospace Engineering

\author{
Morgantown, West Virginia \\ 2017
}

Keywords: dust combustion, mining safety, flame acceleration, Darrieus-Landau instability. Copyright 2017 Sinan Demir 


\title{
Abstract \\ ANALYTICAL AND COMPUTATIONAL STUDIES OF GASEOUS AND DUSTY COMBUSTION: FROM THE IMPACTS OF LOCAL FLAME SPEED VARIATIONS TO A GLOBAL PREDICTIVE MINING FIRE SCENARIO
}

\begin{abstract}
Sinan DEMIR
Accidental fires and explosions in gaseous environments with dust impurities constitute a tremendous hazard for dwelling and office buildings as well as for industries dealing with explosive materials such as flammable gasses and combustible dust. Among these industries, coal mining traditionally exhibits one of the highest occupational fatality and injury rates, often due to methane/air/coal-dust catastrophes claiming many lives every year world wide. There is therefore a critical need to reduce the risk of such accidents, or at least to mitigate their disastrous consequences. For this sake, the analytical and computational studies of the present Dissertation reveal the mechanism of flame evolution and acceleration in a coal mine combustion accident, thereby commanding both the practical relevance mentioned above as well as the fundamental interests. First, the key stages and characteristics of premixed flame front evolution including the flame shapes, propagation speeds, acceleration rates and run-up distances - are scrutinized in two-dimensional planar and cylindrical geometries. Starting with gaseous combustion, the analysis is subsequently extended to gaseous-dusty environments, and the impacts of the size and concentration of the dust particles are quantified. Second, the effect of gas compressibility is incorporated into the theory of a methane/air/coal-dust fire in a mining passage. It is shown that gas compression moderates flame acceleration, and its impact depends on the type of the fuel, its various thermal-chemical parameters as well as the geometry of the problem. Third, spatial variations of laminar flame speed $S_{L}$ are studied to account for the potential impacts of pressure and temperature variations, as well as non-uniform distribution of the equivalence ratio and/or of combustible or inert dust impurities. Specifically linear, parabolic and hyperbolic $S_{L}$-distributions are incorporated into the formulations of "finger" like flame acceleration. The conditions promoting or moderating flame acceleration are identified for these distributions. Finally, gaseous-dusty premixed combustion in a channel, resembling a methane-air fire scenario in a coal mine, is studied by means of computational simulations. The numerical approach employs a finite-volume, Navier-Stokes code solving for the reacting flow equations with fullycompressible hydrodynamics, transport properties, and an Arrhenius chemical kinetics model. The combustible dust particles are incorporated into the solver in such a manner that an "effective fluid", with locally-modified dust-induced flow and flame parameters, replaces a real gaseous-dusty environment. Specifically, flame acceleration due to wall friction is analyzed for linear, parabolic and cubic coal dust concentration spatial distributions. The similarity and differences in the evolutions of the flame morphology and velocity in each of these cases, as well as in the case of purely gaseous combustion, are identified. It is shown that a non-uniform dust distribution may result in an extra distortion or a local stabilization of the flame front, which respectively increases or reduces the total flame surface area, thereby promoting or moderating flame acceleration.
\end{abstract}




\section{DEDICATION}

To my wife Jessica and all my family members. 


\section{ACKNOWLEDGMENTS}

First and foremost, I would like to thank the Creator of entire Universe since nothing is ever possible without him.

I would like to express my genuine appreciation to all great scientists who have shaped our World with their magnificent works and contributions.

I am grateful to my supervisor Dr. V'yacheslav Akkerman for welcoming me to combustion science and giving me the opportunity to complete my dissertation. Without his continuous guidance and support, the completion of this dissertation would not have been possible.

I should also thank to my committee members namely, Drs. Richard Bajura, John Kuhlman, Ali Rangwala and Cosmin Dumitrescu for all their advice and support.

I would like to thank my co-authors Vitaly Bychkov, Sri Chalagalla, Anish Calavay, Hayri Sezer and Torli Bush. I specifically recognize Dr. Vitaly Bychkov, who passed away when several of our joint works were in progress. Many thanks to our collaborator Dr. Damir Valiev for answering my questions about the computational platform. I am also thankful to all our past and present research group members - Orlando Ugarte, Berk Demirgok, Serdar Bilgili, Jad Sadek, Frank Kronz, Swathi Shetti, Afeez Adebiyi, Bugzy Idowu and Rawan Alakandari - for their support and sincere friendship.

This study is sponsored by the US National Science Foundation (NSF) and the Alpha Foundation for the Improvement of Mine Safety and Health, Inc. (ALPHA FOUNDATION) as well as by West Virginia University's Program to Stimulate Competitive Research (PSCoR) and West Virginia University's Senate Award for Research and Scholarship. I am also grateful to West Virginia University Office of Graduate Education \& Life for a Doctoral Scholarship.

I would like to give thanks for their financial support.

I could not forget to thank my family for their presence, care, attention and support. Specifically, I owe a depth of gratitude to my lovely wife Jessica - for her patience and extreme support. My family is the biggest support for me, materially and morally. I will carry you forever in my heart.

Finally, I would like to thank you my reader for your valuable time. 


\section{Table of Contents}

$\begin{array}{lllllllllll}\text { ABSTRACT } \ldots & \ldots & \ldots & \ldots & \ldots & \ldots & \ldots & \ldots & \ldots & \ldots & \text { ii } \\ \text { Dedication } \ldots & \ldots & \ldots & \ldots & \ldots & \ldots & \ldots & \ldots & \ldots & \ldots & \text { iii } \\ \text { Acknowledgements } & \ldots & \ldots & \ldots & \ldots & \ldots & \ldots & \ldots & \ldots & \ldots & \text { iv } \\ \text { List of Figures } & \ldots & \ldots & \ldots & \ldots & \ldots & \ldots & \ldots & \ldots & \ldots & \text { vii } \\ \text { List of Tables } \ldots & \ldots & \ldots & \ldots & \ldots & \ldots & \ldots & \ldots & \ldots & \ldots & \text { xii } \\ \text { List of Symbols } & \ldots & \ldots & \ldots & \ldots & \ldots & \ldots & \ldots & \ldots & \ldots & \text { xiii } \\ \text { List of Papers } \ldots & \ldots & \ldots & \ldots & \ldots & \ldots & \ldots & \ldots & \ldots & \ldots & \text { xvi }\end{array}$

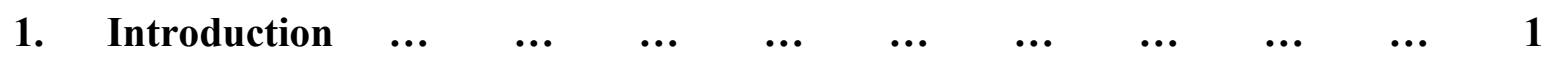

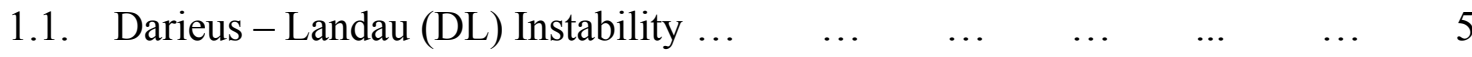

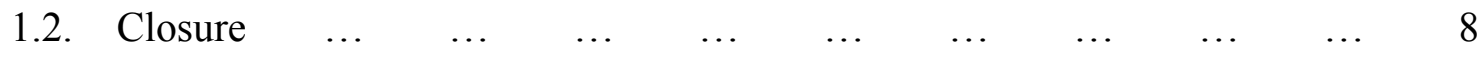

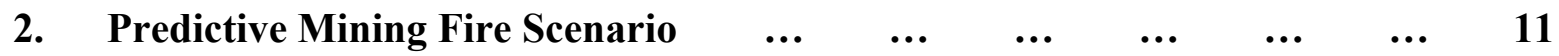

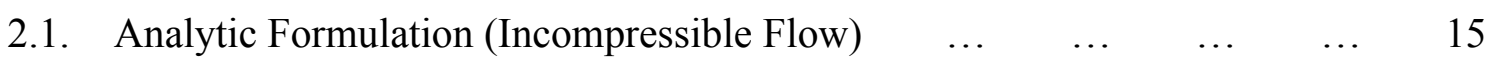

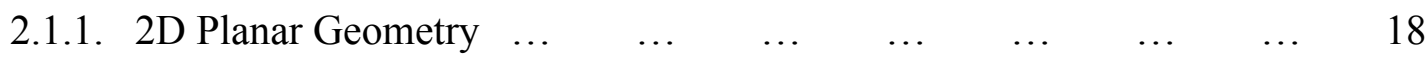

2.1.2. Cylindrical Axisymmetric Geometry $\ldots \begin{array}{llllll}\ldots & \ldots & \ldots & \ldots & \ldots & 20\end{array}$

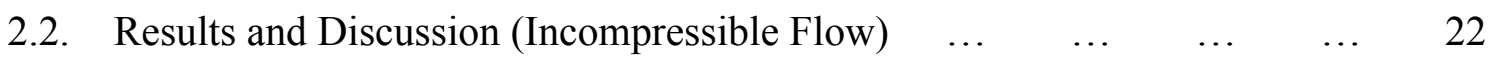

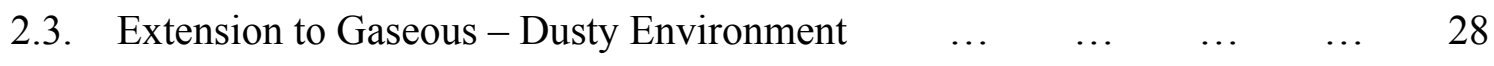

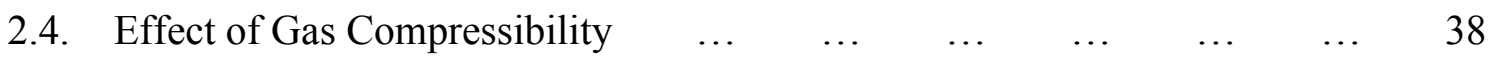

$\begin{array}{lllllllll}\text { 2.4.1. } & \text { 2D Planar Geometry } \\ \ldots & \ldots & \ldots & \ldots & \ldots & \ldots & \ldots & \ldots & 39\end{array}$

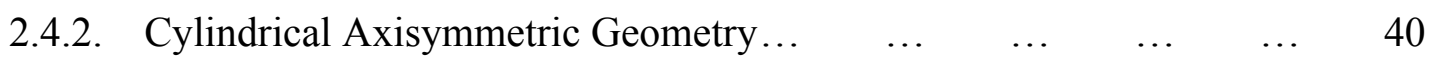

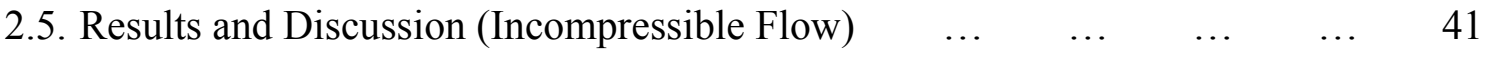

3. Effect of Spatial Variations of the Laminar Flame Speed on the Global

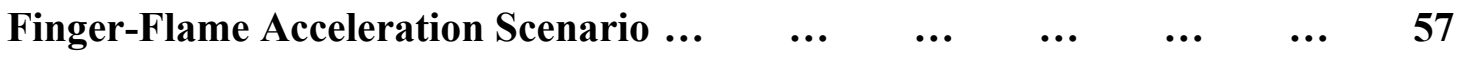

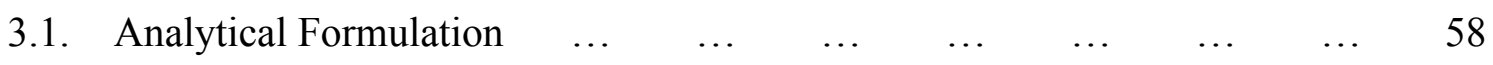

$\begin{array}{llllllllll}\text { 3.1.1. 2D Planar Geometry } & \ldots & \ldots & \ldots & \ldots & \ldots & \ldots & \ldots & 60\end{array}$

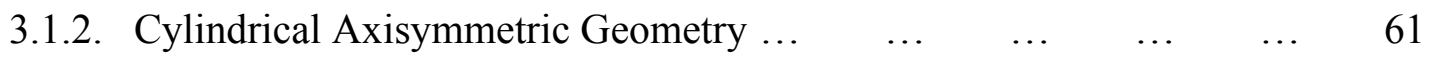

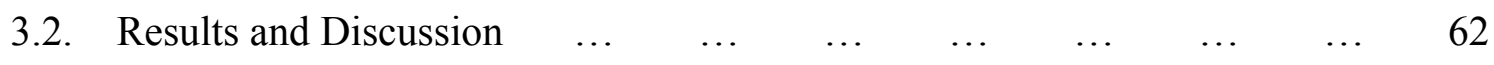




\section{Computational Study of Gaseous - Dusty Combustion with Various Dust}

$\begin{array}{lllllllllll}\text { Distribution } & \ldots & \ldots & \ldots & \ldots & \ldots & \ldots & \ldots & \ldots & \ldots & 68\end{array}$

4.1. Flame Acceleration due to Wall Friction (a.k.a the Shelkin Mechanism) ... 69

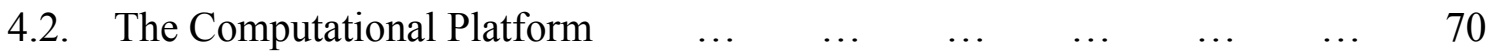

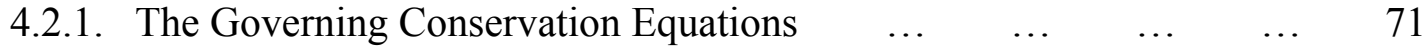

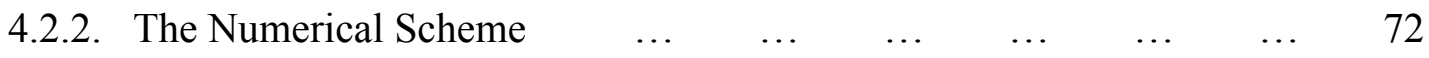

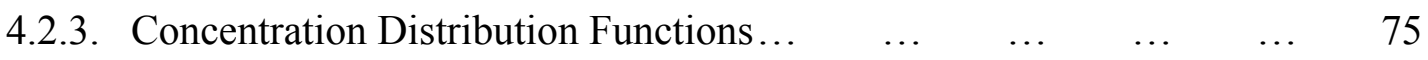

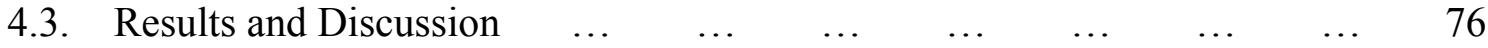

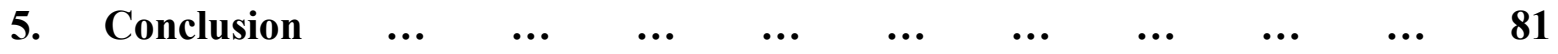

6. $\begin{array}{rlllllllllll}\text { References } & \ldots & \ldots & \ldots & \ldots & \ldots & \ldots & \ldots & \ldots & \ldots & 86\end{array}$

$\begin{array}{lllllllllll}\text { CURRICULUM VITAE } & \ldots & \ldots & \ldots & \ldots & \ldots & \ldots & \ldots & \ldots & 93\end{array}$ 


\section{LIST OF FIGURES}

Figure 1.1: Illustration of premixed flame propagation (a), with the characteristic

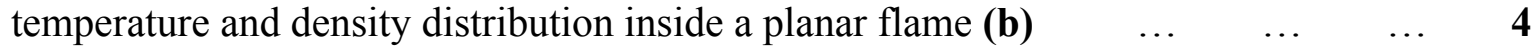

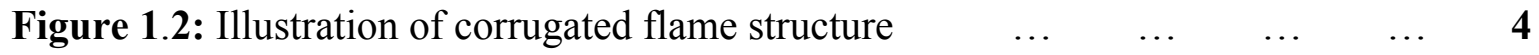

Figure 1.3: Illustration of the Darrieus-Landau $(D L)$ instability (a), and thermal (b) and nonlinear (c) stabilization of the $D L$ instability. Courtesy of $V$. Bychkov

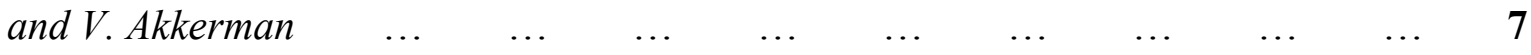

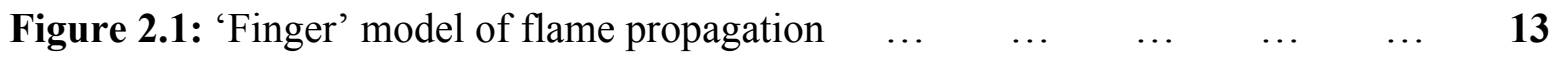

Figure 2.2: Illustration of quasi-spherical flame expansion: the stages of ignition, uniform propagation of a smooth front (a) as well as self-similar

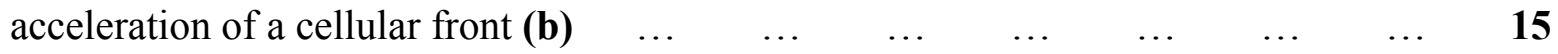

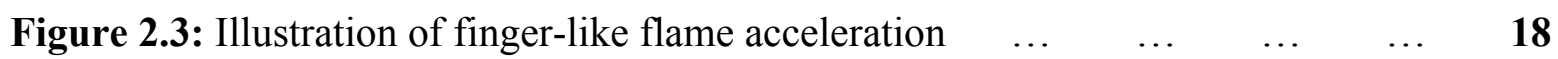

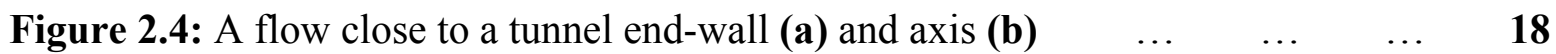

Figure 2.5: $2 D$ planar geometry: The time limitations of finger flame acceleration, $t_{s p h}$, Eq. (2.12), and $t_{\text {wall }}$, Eq. (2.16), versus the equivalence ratio $\phi$ for the propane/air and methane/air flames, $R=H=1.05 \mathrm{~m}, n=1.4 \quad \ldots \quad \ldots$

Figure 2.6: Cylindrical axisymmetric geometry: The time limitations of finger flame acceleration, $t_{s p h}$, Eq. (2.25), and $t_{\text {wall }}$, Eq. (2.29), versus the equivalence ratio $\phi$ for the propane/air and methane/air flames,

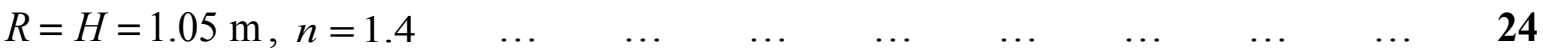

Figure 2.7: (a) Evolution of the flame tip position $Z_{t i p}$ in a stoichiometric $(\phi=1)$ mixture and (b) $Z_{\text {tip }}\left(t_{\text {wall }}\right)$ versus $\phi$ for methane- and propane/air combustion in the $2 \mathrm{D}$ planar and cylindrical axisymmetric geometries $\quad \ldots \quad \ldots \quad \ldots \quad 25$

Figure 2.8: (a) Evolution of the flame tip velocity $U_{t i p}$ in a stoichiometric $(\phi=1)$ mixture and (b) $U_{\text {tip }}\left(t_{\text {wall }}\right)$ versus $\phi$ for methane- and propane/air combustion in the 2D-planar and cylindrical-axisymmetric geometries $\quad \ldots \quad \ldots \quad \ldots$

Figure 2.9: (a) Evolution of the flame tip acceleration $a_{t i p}$ in a stoichiometric $(\phi=1)$ mixture and (b) $a_{\text {tip }}\left(t_{\text {wall }}\right)$ versus $\phi$ for methane- and propane/air combustion in the 2D-planar and cylindrical-axisymmetric geometries $\quad \ldots \quad \ldots \quad \ldots$ 
Figure 2.10: The run-up distance, $Z_{\text {rud }}$, versus the equivalence ratio $\phi$ for methane - air flames, in a cylindrical geometry, and for propane - air flames, in both 2D-planar and cylindrical geometries $\quad \ldots \quad \ldots \quad \ldots$

Figure 2.11: The flame temperature $T_{f}^{*}$ (a) and laminar velocity $S_{d, L}^{*}$

(b) modified by the promotion of the equivalence ratio, versus the particle

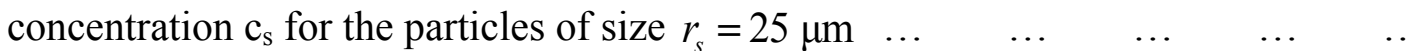

Figure 2.12: The flame temperature $T_{f}^{* *}(\mathbf{a})$ and laminar velocity $S_{d, L}^{* *}$ (b), modified by the heat sink effect, versus the particle concentration $\mathrm{c}_{\mathrm{s}}$ for $\begin{array}{llllllll}\text { the particles of size } r_{s}=25 \mu \mathrm{m} & \ldots & \ldots & \ldots & \ldots & \ldots & \ldots & \ldots\end{array}$

Figure 2.13: The flame temperature $T_{f}^{* * * *}$ (a) and laminar velocity $S_{d, L}^{* * * *}$ (b), modified to the combined (combustible + inert) effect, versus the particle

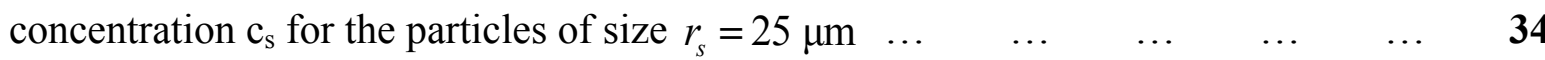

Figure 2.14: The modified flame velocity versus the particle concentration for the fixed equivalence ratio $(\phi=0.7)$ and various particle radii ( $r_{s}=10 ; 25$ and $75 \mu \mathrm{m}$ ) for combustible (a) and combined (combustible $\begin{array}{lllllllllll}+ \text { inert) }(\text { b) } \text { particles } & \ldots & \ldots & \ldots & \ldots & \ldots & \ldots & \ldots & \ldots & \ldots & 35\end{array}$

Figure 2.15: Evolution of flame tip position $Z_{t i p}$ in a homogeneous gaseous environment and in the presence of combustible, inert, and combined (combustible + inert) particles in both 2D-palnar and cylindrical geometries

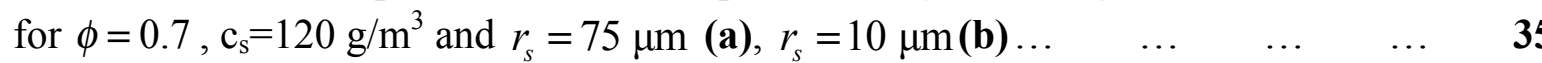

Figure 2.16: Evolution of flame tip position $U_{t i p}$ in a homogeneous gaseous environment and in the presence of combustible, inert, and combined (combustible + inert) particles in both 2D-planar and cylindrical geometries

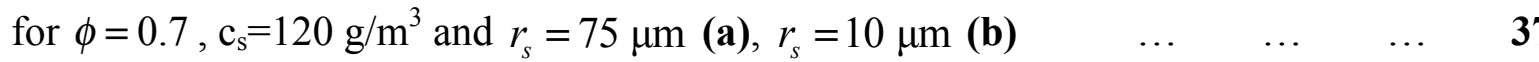

Figure 2.17: Instantaneous flame tip velocities $U_{t i p}\left(t_{s p h}\right)$ and $U_{\text {tip }}\left(t_{\text {wall }}\right)$ versus the particle concentration $c_{\mathrm{s}}$ for combustible, inert and combined particles for $\phi=0.7$ and $r_{s}=25 \mu \mathrm{m}$ in the 2D-planar (a) and cylindrical-axisymmetric (b) geometries

Figure 2.18: 2D-planar geometry: Comparison of the incompressible and compressible formulations: Evolution of the flame tip position $Z_{t i p}$ 
(a) and its velocity $U_{t i p}$ (b) for the methane/air flames of various

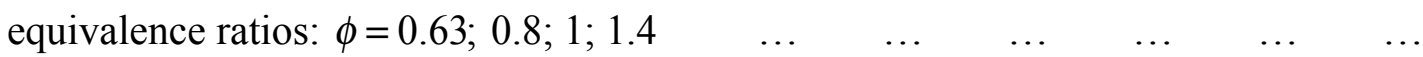

Figure 2.19: 2D-planar geometry: Comparison of the incompressible and compressible formulations: Evolution of the flame tip position $Z_{t i p}$ (a) and its velocity $U_{t i p}$ (b) for the propane/air flames of various

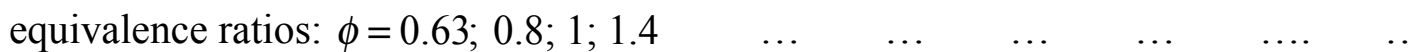

Figure 2.20: Cylindrical-axisymmetric geometry: Comparison of the incompressible and compressible formulations: Evolution of the flame tip position $Z_{t i p}$ (a) and its velocity $U_{t i p}$ (b) for methane/air flames of various

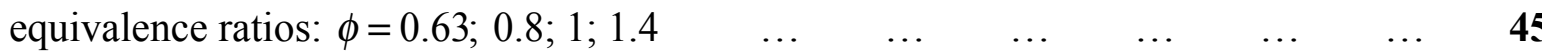

Figure 2.21: Cylindrical-axisymmetric geometry: Comparison of the incompressible and compressible formulations: Evolution of the flame tip position $Z_{t i p}$ (a) and its velocity $U_{t i p}$ (b) for the propane/air flames of various

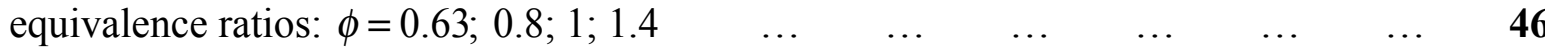

Figure 2.22: Comparison of the incompressible and compressible formulations for the 2D planar and cylindrical geometries: Evolution of the flame tip position $Z_{t i p}$ (a) and its velocity $U_{t i p}$ (b) for the lean $(\phi=0.6)$ and rich $(\phi=1.4)$ methane/air flames

Figure 2.23: Comparison of the incompressible and compressible formulations for the 2D-planar geometry: Evolution of the flame tip position $Z_{t i p}$ (a) and its velocity $U_{t i p}$ (b) for the methane/air flames of the equivalence ratio $\phi=0.7$ in the presence of combustible dust of the concentration $\mathrm{c}_{\mathrm{s}}=120 \mathrm{~g} / \mathrm{m}^{3}$ and the

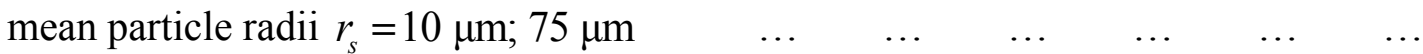

Figure 2.24: Comparison of the incompressible and compressible formulations for cylindrical geometry: Evolution of the flame tip position $Z_{t i p}$ (a) and its velocity $U_{t i p}$ (b) for the methane/air flames of the equivalence ratio $\phi=0.7$ in the presence of combustible dust of the concentration $\mathrm{c}_{\mathrm{s}}=120 \mathrm{~g} / \mathrm{m}^{3}$ and the

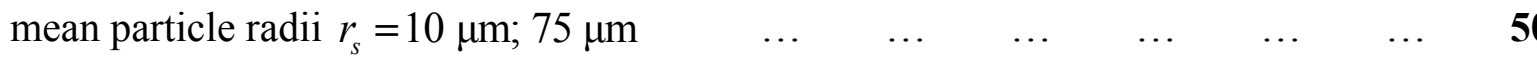

Figure 2.25: Comparison of the incompressible and compressible formulations for the 2D-planar and cylindrical-axisymmetric geometries: the maximal coordinate $Z_{\text {tip }}\left(t_{\text {wall }}\right)(\mathbf{a}, \mathbf{c})$ and velocity $U_{\text {tip }}\left(t_{\text {wall }}\right)(\mathbf{b}, \mathbf{d})$ versus the combustible $(\mathbf{a}, \mathbf{b})$ and inert $(\mathbf{c}, \mathbf{d})$ particle concentration for $\phi=0.7$ and the mean particle 
radii $r_{s}=25 \mu \mathrm{m}$

Figure 2.26: Comparison of the incompressible and compressible formulations for the 2D-planar geometry: Evolution of the flame tip position $Z_{t i p}$ (a) and its velocity $U_{t i p}$ (b) for the methane/air flames of equivalence ratio $\phi=0.7$ in the presence of the combustible, inert and combined particles of the concentration $\mathrm{c}_{\mathrm{s}}=120 \mathrm{~g} / \mathrm{m}^{3}$ and the mean particle radius $r_{s}=25 \mu \mathrm{m} \ldots$

Figure 2.27: Comparison of the incompressible and compressible formulations for the cylindrical-axisymmetric geometry: Evolution of the flame tip position $Z_{t i p}$ (a) and its velocity $U_{t i p}$ (b) for the methane/air flames of the equivalence ratio $\phi=0.7$ in the presence of combustible, inert and combined particles of the concentration $\mathrm{c}_{\mathrm{s}}=120 \mathrm{~g} / \mathrm{m}^{3}$ and the mean particle radius $r_{s}=25 \mu \mathrm{m} \quad \ldots \quad \ldots \quad \ldots$

Figure 2.28: Comparison of the incompressible and compressible formulations for the 2D-planar and cylindrical-axisymmetric geometries: evolution of the flame tip position $Z_{t i p}$ (a) and its velocity $U_{t i p}$ (b) for the methane/air flames of the equivalence ratio $\phi=0.7$ in the presence of the combustible, inert and combined particle of the concentration $\mathrm{c}_{\mathrm{s}}=120 \mathrm{~g} / \mathrm{m}^{3}$ and the mean particle $\begin{array}{llllllllll}\text { radius } r_{s}=25 \mu \mathrm{m} & \ldots & \ldots & \ldots & \ldots & \ldots & \ldots & \ldots & \ldots & \ldots\end{array}$

Figure 3.1: Schematic of various $S_{L}$ - distributions, Eq. (3.1): (a) constant, (b) linear, (c) tangential-hyperbolic, (d) parabolic (promotion along the axis, $a=1, \varsigma=0.8$ ), and (e) parabolic (promotion through wall, $a=0.2, \varsigma=-0.8$ ) $\quad \ldots$

Figure 3.2: $2 D$ geometry: Evolution of the scaled flame skirt position (a), flame tip position (b), flame tip velocity (c), and flame tip acceleration (d) versus scaled time, $\tau=t \times S_{L}^{0} / R$, for the constant, liner, hyperbolic and parabolic $\begin{array}{lllllllllll}S_{L} \text { - distributions } & \ldots & \ldots & \ldots & \ldots & \ldots & \ldots & \ldots & \ldots & \ldots\end{array}$

Figure 3.3: Axisymmetric geometry: Evolution of the flame skirt position (a), flame tip position (b), flame tip velocity (c), and flame tip acceleration (d) versus scaled time, $\tau=t \times S_{L}^{0} / R$, for the constant, liner, hyperbolic and parabolic $S_{L^{-}}$ $\begin{array}{llllllllllll}\text { distributions } & \ldots & \ldots & \ldots & \ldots & \ldots & \ldots & \ldots & \ldots & \ldots & \ldots & 64\end{array}$

Figure 3.4: Comparison of the 2D-planar and cylindrical axisymmetric cases for the constant and parabolic velocity distributions: Evolution of the flame tip position (a) and its velocity (b) versus scaled time, $\tau=t \times S_{L}^{0} / R \quad \ldots \quad$... 
Figure 3.5: 2D geometry: Evolution of the flame tip position (a) and velocity

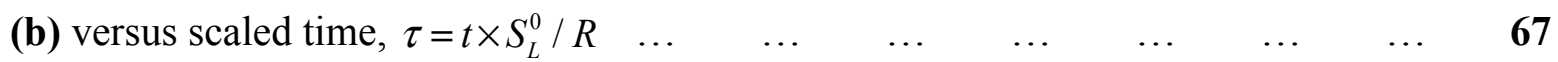

Figure 4.1: Flame acceleration due wall friction (a.k.a the Shelkin mechanism) $\quad \ldots \quad \mathbf{7 0}$

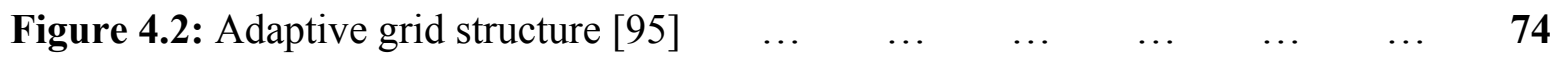

Figure 4.3: Effect of four different flame grid size on the scaled flame tip

$\begin{array}{llllllllllll}\text { Position }[57] & \ldots & \ldots & \ldots & \ldots & \ldots & \ldots & \ldots & \ldots & \ldots & \ldots & 74\end{array}$

Figure 4.4: Schematic of the different coal dust concentration distributions: (a)

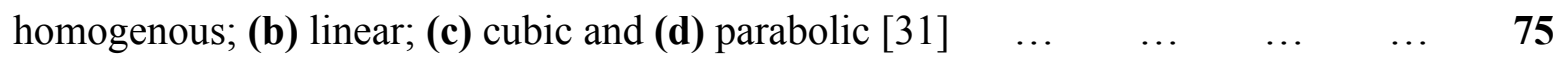

Figure 4.5: The scaled flame velocity at the centerline $U_{c} / S_{L}$ versus the scaled

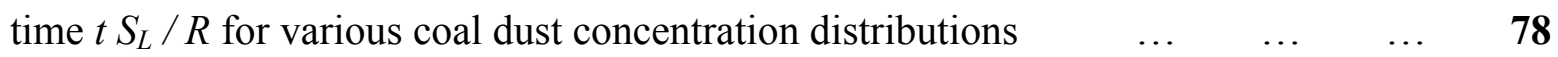

Figure 4.6: The scaled flame surface area $A_{\mathrm{w}} / 2 R$ versus the scaled time $t S_{L} / R$

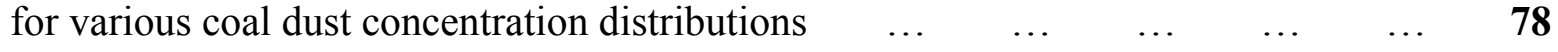

Figure 4.7: Temperature snapshots at time: $\tau=0$ (a), 0.15 (b), 0.27 (c), 0.36

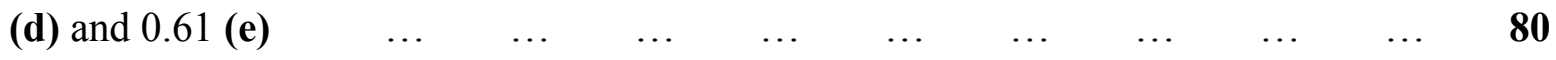




\section{LIST OF TABLES}

Table 2.1: Various mechanisms of flame corrugation/acceleration in

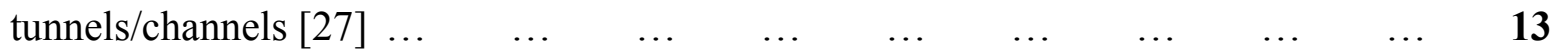

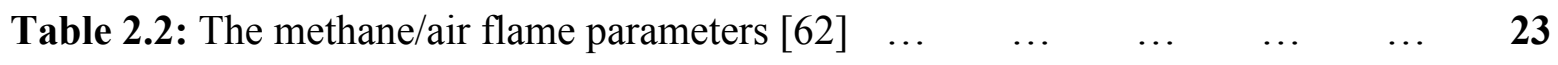

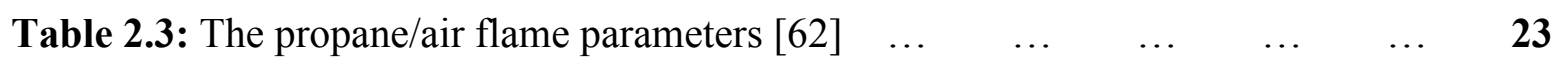

Table 2.4: Some physical parameters used in the study [67] $\quad \ldots \quad \ldots \quad \ldots \quad \ldots \quad 31$ 


\section{LIST OF SYMBOLS}

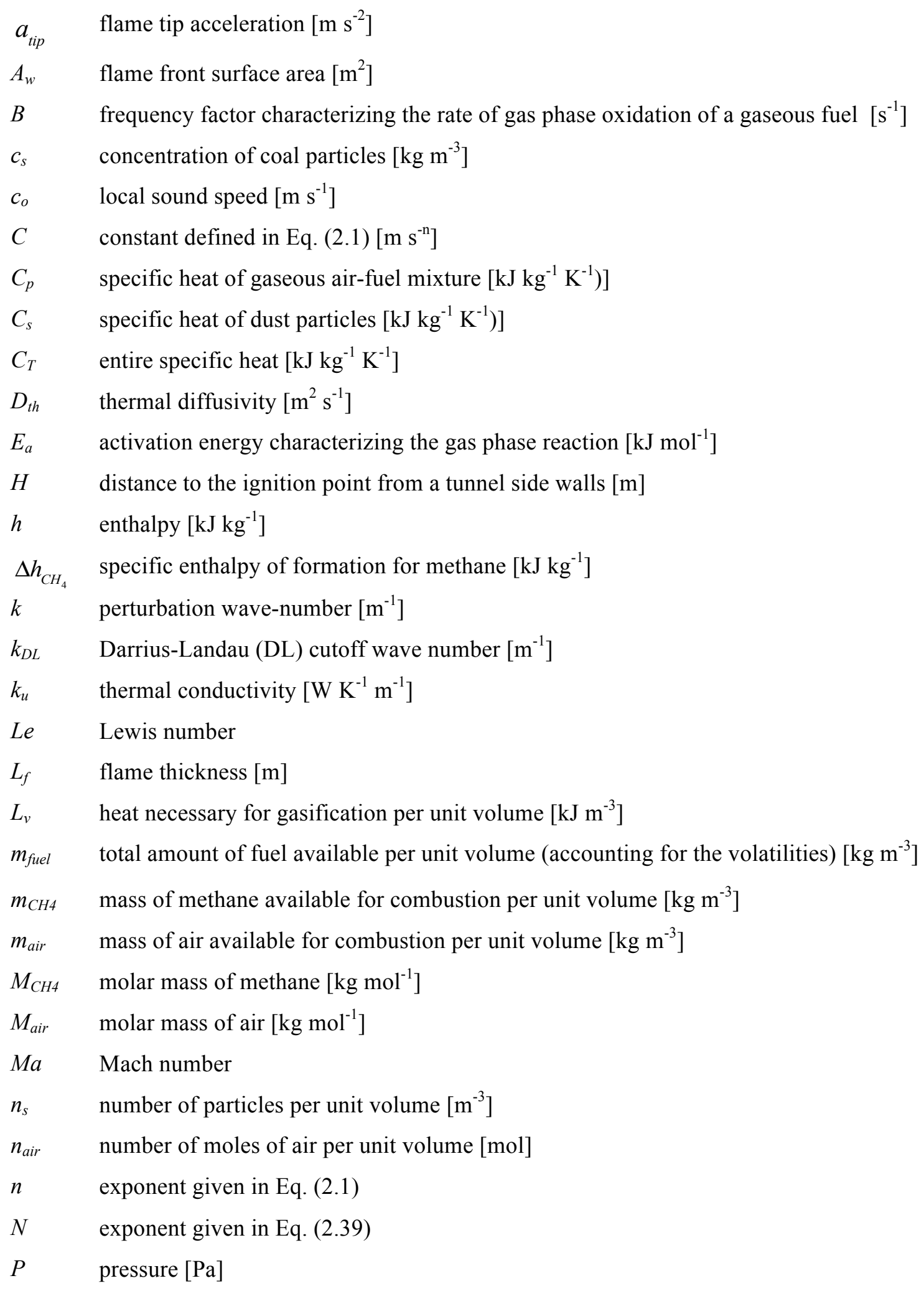




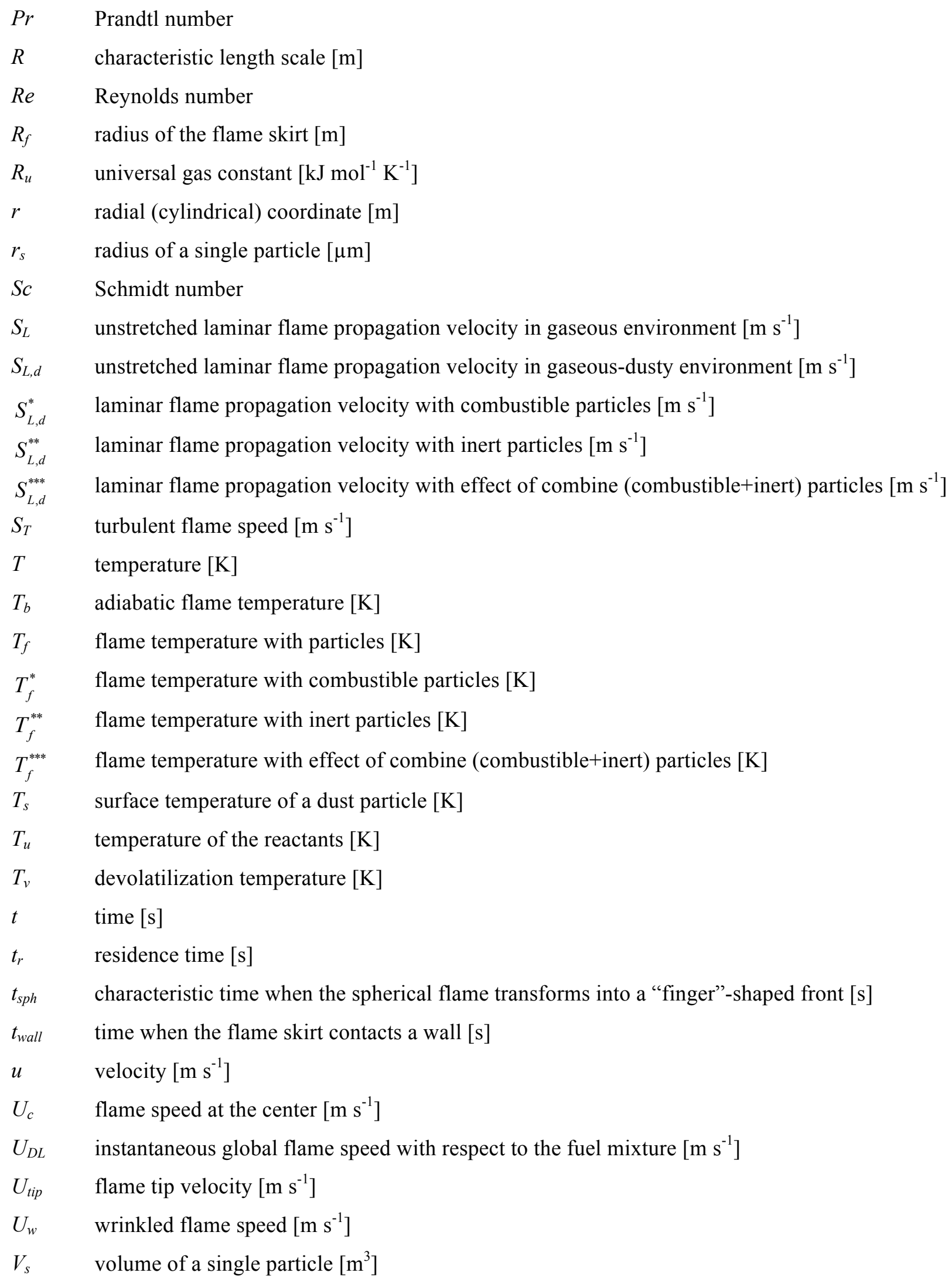




$\begin{array}{ll}v & \text { kinematic viscosity }\left[\mathrm{m}^{2} \mathrm{~s}^{-1}\right] \\ Y & \text { mass fraction } \\ x & \text { radial (2D) coordinate }[\mathrm{m}] \\ Z e & \text { Zeldovich number } \\ Z_{\text {rud }} & \text { run-up-distance }[\mathrm{m}] \\ Z_{t i p} & \text { flame tip position }[\mathrm{m}] \\ z & \text { axial coordinate }[\mathrm{m}] \\ Q & \text { heat released during combustion per unit volume }\left[\mathrm{kJ} \mathrm{m}^{-3}\right] \\ w_{v}^{\prime} & \text { devolatalization rate }\left[\mathrm{kg} \mathrm{m}^{-3} \mathrm{~s}^{-1}\right] \\ w_{v} & \text { total mass of volatilities released per unit volume }\left[\mathrm{kg} \mathrm{m}^{-3}\right]\end{array}$

Greek Symbols

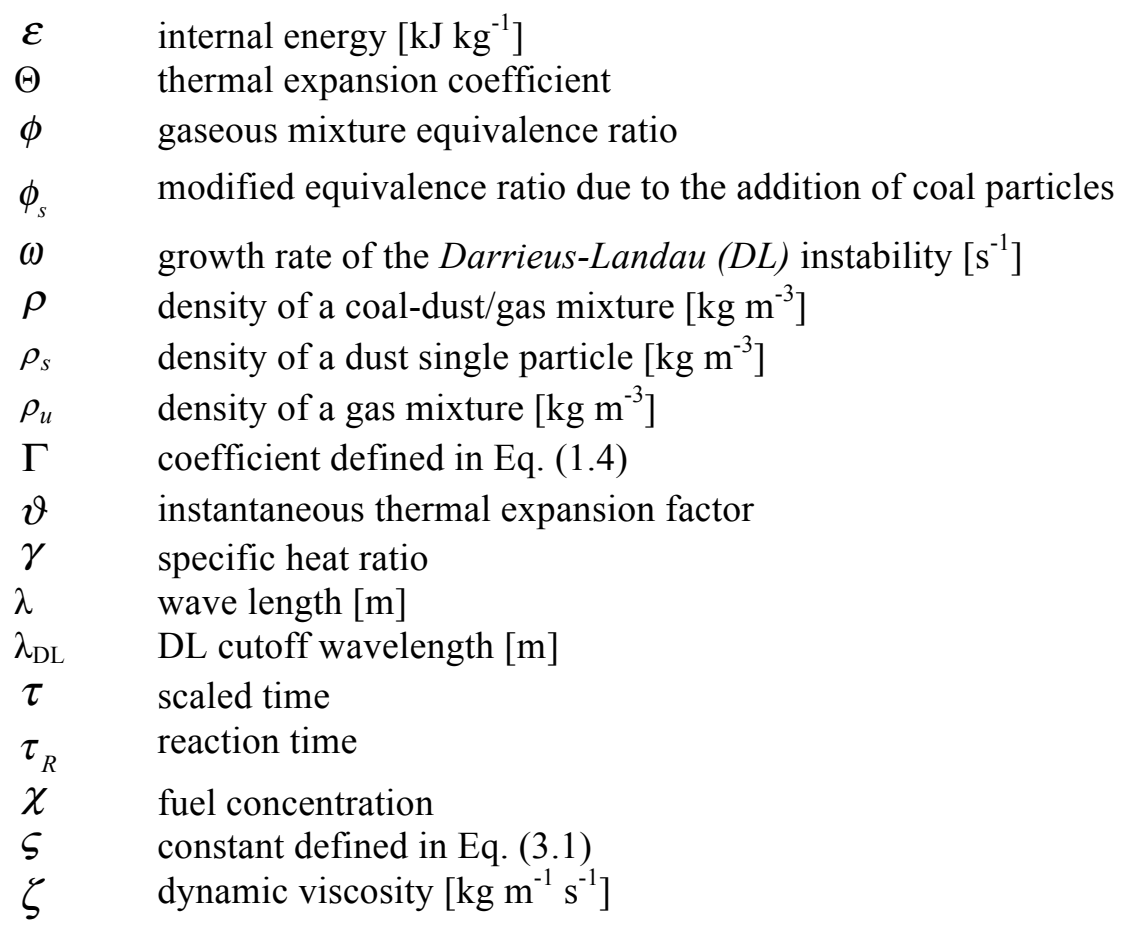

\section{Subscripts}

$s \quad$ coal dust particle

$u \quad$ ambient conditions

$0 \quad$ initial conditions

1 air - fuel mixture

2 burnt matter 


\section{LIST OF PAPERS}

\section{The present Dissertation is based on the following refereed papers, as cited in the text.}

1. Demir S., Bychkov V., Chalagalla S.H.R., Akkerman V., Towards Predictive Scenario of a Burning Accident in a Mining Passage, Combustion Theory and Modelling, 2017Published online.

2. Demir S., Calavay A.R., Akkerman V., Influence of Gas Compressibility on a Burning Accident in a Mining Passage, Combustion Theory and Modelling, 2017-Accepted.

3. Demir S., Sezer H., Akkerman V., Effect of Local Variations of the Laminar Flame Speed on the Global Finger-Flame Acceleration Scenario, Combustion Theory and Modelling, 2017-under review.

4. Demir S., Sezer H., Bush T., Akkerman V., Promotion and Mitigation of Premixed Flame Acceleration in Dusty-Gaseous Environment with Various Combustible Dust Distributions: A Computational Study. 26th International Colloquium on the Dynamics of Explosions and Reactive Systems (ICDERS 2017), July 30-August 4, Boston-USA, pp. $1-6$.

\section{List of papers not included in the present Dissertation}

1. Bilgili S., Bychkov V., Morella H., Demir S., Valiev D., Akkerman V., Impact of the Lewis and Markstein Numbers Effects on Flame Acceleration in Channels, submitted.

2. Akkerman V., Demirgok B., Demir S., Bychkov V., Valiev D., Law C.K., Wu M.H., Acceleration of Ethylene-Oxygen Flames in Micro-Pipes: Theory, Experiment and Modelling, submitted. 


\section{Introduction}

Combustion is one of the oldest known sources of energy; it has been used over millennia for different purposes. Heating, cooking, transportation and energy production are the basic human activities that have widely employed combustion. Currently, approximately $90 \%$ of the world's energy production depends on the combustion processes [1]. It is widely accepted that despite the modern revolution in alternative energy sources and their utilization, this trend will continue in the foreseeable future because of its convenience, high-energy density, as well as the economic and political situation of the world [2].

While it is a useful tool, combustion can also be catastrophic disaster. For instance, wild land and urban fires, as well as explosions in power plants and coal mines are the dark side of the usage of combustion that mankind unavoidably faces. In this respect, controlling sudden fires and explosions and protecting people from their consequences are equally important as the facilitation of combustion processes. Indeed, the dual-sided nature of combustion, its usefulness and high risk potential, has made it a hot topic for many researchers.

Combustion is a chemical reaction that occurs between two reactants, a fuel and an oxidizer generally at high temperatures. Common fuels are hydrogen and hydrocarbons, which include methane, propane, wood, coal, etc. Typical oxidizers are the environmental air (nitrogen+oxygen), pure oxygen, or sometimes fluorine in the rocketry applications. Combustion processes are also categorized in terms of the mixing of the fuel and oxidizer before or after the ignition. Namely, if the fuel and oxidizer are perfectly mixed before ignition, the burning process is called premixed combustion. In contrast, in a nonpremixed system, the fuel and the oxidizer remain separated prior to their involvement into the reaction zone. Premixed gaseous combustion occurs in engines, gas turbines, appliances and explosions. Here combustion occurs by means of the propagation of a front separating the unburned fuel-oxidizer mixture from the fully burned combustion products [3]. On the other hand, candles or diesel engines are one of the most commonly used applications of non-premixed combustion. This dissertation is limited to premixed combustion. 
Two classes of self-sustained waves of the exothermic chemical reaction spreading through a homogeneous combustible premixture can be distinguished as a subsonic deflagration (flame) or a supersonic detonation wave. Both the deflagration and detonation appear to be stable attractors each being linked to its own base of initial data [3].

In a detonation, a reaction propagates due to rapid shock and compression waves. In the deflagration (flame) mode, a chemical reaction propagates due to thermal conduction and diffusion. Figure 1.1 illustrates the characteristic internal structure of a planar flame when a fresh fuel mixture is ignited. Here, some distinctive regions are marked with different colors. In the unburned region (the fuel-oxidizer mixture), the reaction has not started yet, while in the burnt matter region the reaction is already completed. The "flame front" is actually a narrow band between them. The hot burnt gas heats the fuel-oxidizer mixture by heat conduction, mainly, in the reaction zone, hence initiating the chemical reaction in the neighboring areas. This type of combustion shows a remarkable dependence of the reaction rate versus temperature, e.g. a twofold increase in the fuel temperature might lead to a 10-12 orders of magnitude increase in the reaction rate [4].

The main flame parameters are the thermal expansion factor $\Theta$ defined as a fresh to burnt gas density ratio $\Theta=\rho_{u} / \rho_{f}$ being about 5-10 for typical hydrocarbons [5]. By assuming isobaric approximation and constant molar weights, it can be also defined as $\Theta=T_{f} / T_{u}$. With respect to a fuel mixture, a planar premixed flame front, is shown schematically in Fig. 1.1, would propagate with an unstretched laminar speed, $S_{L}$, which is a thermal-chemical parameter. Various definitions of flame speed can be found in the literature, and this might sometimes be quite bewildering. This question is elucidated quite well in Ref. [6]. Once the flame speed $S_{L}$ is known, the thermal-diffusion flame thickness $L_{f}$ may be estimated conventionally as

$$
L_{f}=\frac{k_{u}}{C_{P} \rho_{f} S_{L}}
$$

where $k_{u}$ is the thermal conduction coefficient and $C_{P}$ is the specific heat at constant pressure. However, the result (1.1) is a just a characteristic length scale, determined by the transport 
properties; it may differ from the "real flame thickness" by an order of magnitude or thereabout [7]. Typical values of $S_{L}$ and $L_{f}$ are $\left(10^{-2}-10^{-1}\right) \mathrm{m}$ and $\left(10^{-1}-10\right) \mathrm{m} / \mathrm{s}$, respectively.

However, a planar flame front of seen Fig. 1.1 rarely (if ever) occurs in practical reality. Indeed, the majority of industrial and laboratory flames are usually corrugated due to turbulence [8-10], acoustics [11-13], shocks [14, 15], combustion instabilities [5, 16], wall friction [17, 18], or in-built obstacles $[19,20]$. A curved flame front, shown in Figure 1.2, has a larger surface area relative to a planar one; therefore, it consumes more fuel mixture per unit time and releases more heat, thereby propagating faster than the planar flame front, say, with a certain "wrinkled" flame speed $U_{w}$.

As stated above, a deflagration is initiated by an energy release, such as a spark, while detonation is activated by shock waves via localized explosions. Detonations can be generated directly if a large amount of energy is rapidly released in the explosive mixture. Yet, it has long been known that the initially formed deflagration undergoes acceleration accompanied by a rapid change in the velocity of the reaction wave, when it is followed by a transition from a deflagration to a detonation mode [3]. Such a deflagration-to-detonation transition (DDT) is a fundamental and intriguing combustion problem, and is considered one of the most relevant unsolved problems in modern combustion science [21]. The underlined differences between deflagration and detonation regimes make the DDT both a useful and potentially catastrophic phenomenon, especially in those scenarios where it has not been predicted or controlled such as accidental gaseous and dusty explosions in coal mines. However, DDT has a great technological potential as observed in propulsion system applications and in technologies such as pulse detonation engines. It can also be observed extra terrestrially in the form of supernova explosions. 


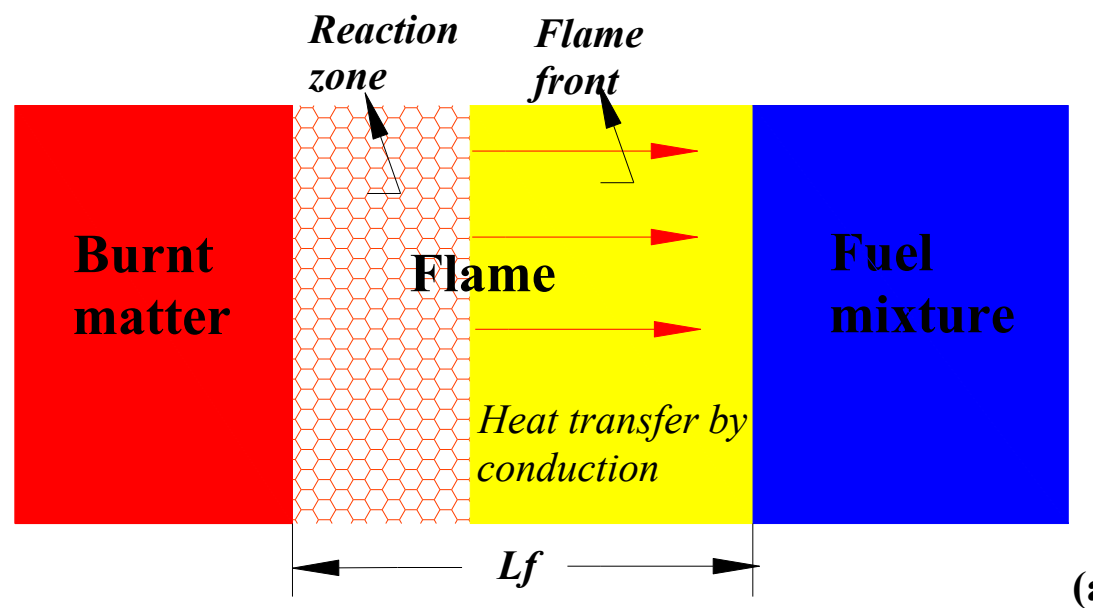

(a)

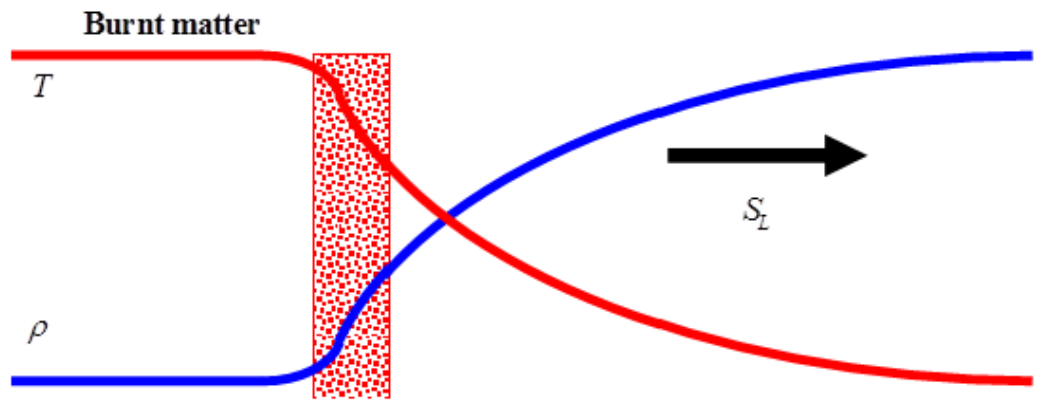

Unburnt matter

(b)

Figure 1.1: Illustration of premixed flame propagation (a), with the characteristic temperature and density distribution inside a planar flame (b).

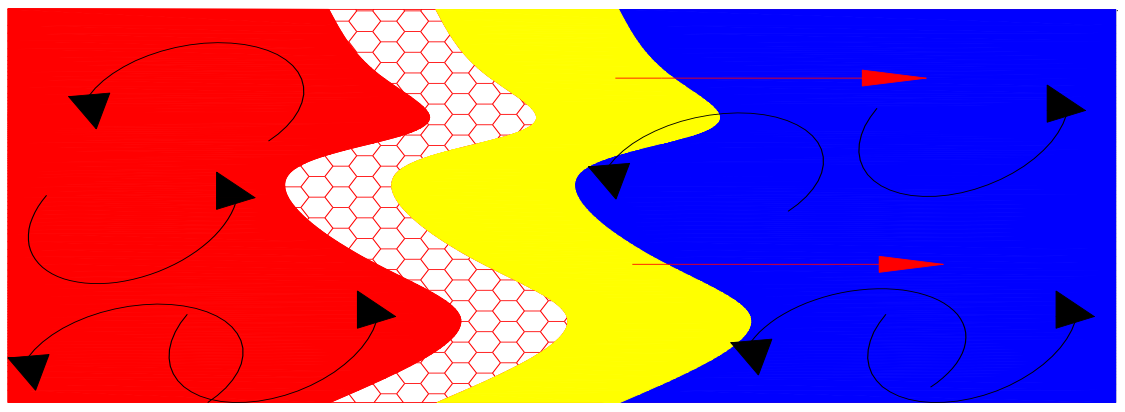

Figure 1.2: Illustration of corrugated flame structure. 


\subsection{The Darrieus - Landau (DL) Instability}

The theory of hydrodynamic stability of propagating flames was developed by Darrieus [22] and Landau [23] and is known as the DL theory. Such instability is driven by gas expansion in exothermic reactions. A planar flame may experience the DL instability, and as a result eventually becomes corrugated (sometimes non-stationary and turbulent). Their analyses, based on an infinitely thin flame front, showed that a propagating planar flame in a gaseous mixture is intrinsically unstable against any perturbations that bend the flame front. The perturbation is assumed to be in the form [5]:

$$
\tilde{F}(x, t)=\tilde{F}(x) \exp (\omega t+i k x),
$$

where $k$ is the wave number of the perturbation ( $k=2 \pi / \lambda$ and $\lambda$ is the wavelength), $x$ is the coordinate axis along the flame front, $\omega$ is the growth rate of the perturbation in time as a function of the wave number $(\omega=\omega(k))$ and $\omega$ is found as a solution of dispersion relation. By considering simple dimensional analysis one would expect the growth rate in the following form: $\omega=\Gamma(\Theta) S_{L} k$, where $\Gamma(\Theta)$ is the numerical term. For the sake of brevity, all detailed derivations will not be presented, but one can find the details of algebraic equations in Ref. [5]. Thus, dispersion relation and growth rate is derived respectively for a $2 D$ configuration as:

$$
\begin{aligned}
& \omega^{2}+[2 \Theta /(\Theta+1)] S_{L} k \omega-\Theta[\Theta-1 /(\Theta+1)] S_{L}^{2} k^{2}=0, \\
& \omega / S_{L} k=\Gamma(\Theta)=\frac{\Theta}{\Theta+1}\left[\left(\Theta+1-\frac{1}{\Theta}\right)^{1 / 2}-1\right] .
\end{aligned}
$$

Simply, Eqs. (1.2)-(1.4) state that without gas expansion there is no instability, and the instability mode depends on a thermal expansion degree (the larger $\Theta$, the stronger instability). In contrast, varying perturbation wavelengths grow in time at different rates: the larger the perturbation wavelength (small $k$ ), the slower it grows and vice versa.

As a result, in the $D L$ instability, as illustrated schematically in Fig. 1.3(a), small perturbations imposed to a zero thickness flame front (mathematical surface) growing exponentially in time, bend the flame front with the magnitude of the growth rate $\omega$ and the 
flame front moves with a local constant speed $S_{L}$, with respect to fuel mixture, where $S_{L}$ is considered as an external parameter. The $D L$ instability mode is valid if the characteristic length scale of the problem, in this case $\lambda$, is much larger than the thermal flame thickness $\left(\lambda \gg L_{f}\right)$.

When the $\lambda$ becomes comparable to $L_{f}$ in two orders of magnitude, which is the case of short wave length perturbations, one shall change the statement of the problem from the beginning and re-drive the perturbation growth rate by considering a finite flame thickness. In this case, thermal conduction and diffusion processes play a significant role in the flame dynamics and structure. The rigorous linear theory of the $D L$ instability in the case of small but finite flame thickness was developed by Pelce and Clavin [24]. Assuming a unity Lewis number Le (the thermal-to-mass diffusivities ratio), no gravitational force and constant transport properties, the dispersion relation and instability grow rate take the form of

$$
\begin{aligned}
& \omega^{2}+\frac{2 \Theta}{\Theta+1}\left(1+\frac{\Theta \ln \Theta}{\Theta-1} k L_{f}\right) S_{L} k \omega-\Theta \frac{\Theta-1}{\Theta+1}\left[1-k L_{f}\left(1+\Theta \ln \Theta \frac{\Theta+1}{(\Theta-1)^{2}}\right)\right] S_{L}^{2} k^{2}=0 \\
& \omega=\Gamma(\Theta) S_{L} k=\left(1-\frac{k \lambda_{D L}}{2 \pi}\right)
\end{aligned}
$$

where $\Gamma(\Theta)$ is the same as in. Eq. (1.4) and $\lambda_{D L}$ is the critical cut-off wavelength, when it defines the criteria for the stabilization mode due to thermal conduction. The latter, in turn, depends on $\Theta$ and is proportional to the flame thickness $L_{f}$ as $\lambda_{D L} \approx(30-100) L_{f}$ for $\Theta=5-8 . \lambda_{D L}$ also determines the critical length scale, for which the instability may develop. In the case of zero flame thickness, Eq. (1.5) reduces to Eq. (1.3).

As illustrated in Figure 1.3(b), heat fluxes in the flame front and converges in the convex region, thereby providing effective heating, which results in the increase of the local flame propagation speed $S_{L}$. If $\lambda>\lambda_{D L}$, the perturbations will continue to grow until the nonlinear stabilizing effects become important.

The nonlinear mechanism of flame stabilization, which works according to Huygens' Principle, is illustrated in Figure 1.3(c). This mechanism of flame stabilization was initially proposed by Zeldovich [25]. Specifically, since initially sinusoidal flame front propagates 
normal to itself, concave parts of the flame front converge while convex parts diverge. Thus, the flame velocity increases at the cusp region and the flame propagates faster therein, which due to $D L$ instability, balances the growth and amplitude of the humps, resulting in a curved stationary shape at the end due to non-linear effects. When a flame front has a finite thickness, thermal conduction would smooth the cusps [3].
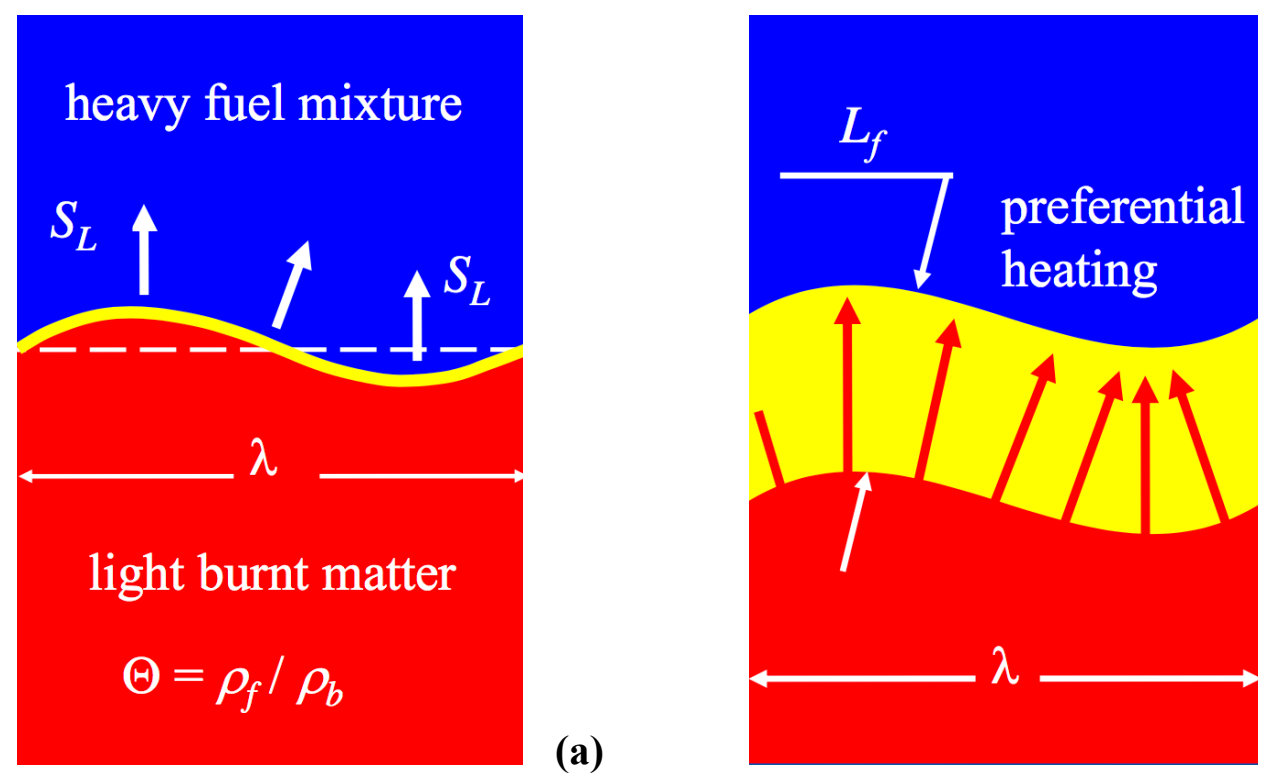

(b)

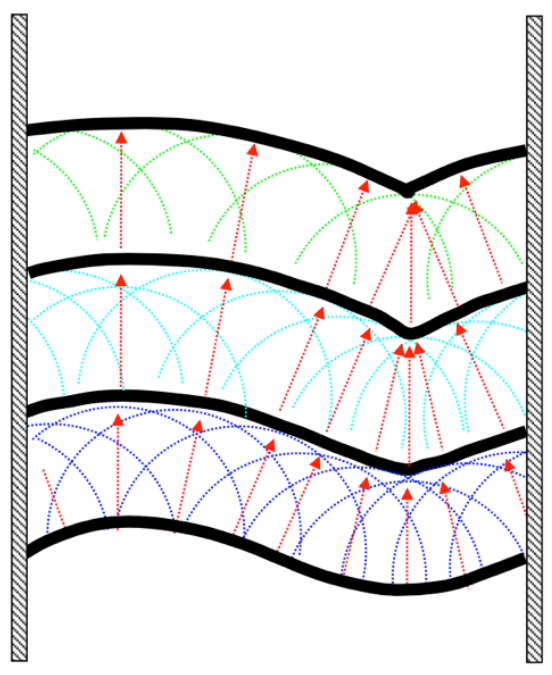

(c)

Figure 1.3: Illustration of the Darrieus-Landau $(D L)$ instability (a), and thermal (b) and nonlinear (c) stabilization of the $D L$ instability. Courtesy of $V$. Bychkov and $V$. Akkerman. 


\subsection{Closure}

Combustion is a beautiful yet challenging branch of modern science, which has not been fully understood or solved. Moreover, it is an interdisciplinary topic, which includes thermodynamics, fluid mechanics, heat transfer, chemical kinetics and transport phenomena. The complexity of its nature generally consists of having non-linear terms and equations, as well as unsteady and multiphase flows. The development of combustion science has mainly taken place by making logical assumptions and simplifications according to the problem type. For instance, considering one step reaction, zero flame thickness, isobaric approximation, unity Lewis number, taking diffusion and thermal conductivity coefficients as equal as in Zel'dovich-FrankKamentskii flame propagation theory [26] and so on. In this regard, progress in combustion has been initiated with very simple yet logical considerations, and it has been stepped up by improving these theories further. In this respect, this dissertation expands upon previous developed theoretical and computational studies as well as develops original ideas by taking into account safety and controlling mechanisms of combustion.

This dissertation is organized as follows.

(i) Development of a Predictive Mining Fire Scenario - (Papers 1 and 2) - scrutinized the inner mechanisms of a combustion accident in a coal mine. Specifically, the key stages and characteristics of a mining fire scenario are predicted, and the dynamics of a premixed flame front are scrutinized including the evolution of such parameters as the flame shape, propagation speed, acceleration rate and run-up distances. The theories of globally spherical, expanding flames, and finger-flame acceleration are combined into a general analytical formulation. Twodimensional (2D) and to cylindrical mining passages are studied. Starting with gaseous combustion, the analysis is subsequently extended to gaseous-dusty environments. Specifically, combustible dust (e.g. coal), inert dust (e.g. sand), and their combination are considered, and the influence of the size and concentration of the dust particles is quantified. In the following section, the predictive scenario of a methane - air - coal-dust fire in a mining passage [27] is extended by incorporating the effect of gas compressibility in the analysis [28].

(ii) Investigation of the Effect of Local Variations of the Laminar Flame Speed on the Global Finger-Flame Acceleration Scenario - (Paper 3) - In this section, a systematic study of the 
effect of local $S_{L}$ - variations on the global flame evolution scenario was conducted from a fundamental point of view. In practice, $S_{L}$ may vary spatially and temporally due to potential impacts of pressure and temperature variations, as well as non-uniform distribution of the equivalence ratio in a combustor and/or of combustible or inert dust impurities in a coal mine. The spatial variations are assumed to be externally imposed, in a manner being a free functional of the formulation. Specifically, linear, parabolic and hyperbolic spatial $S_{L}$-distributions are incorporated into the formulations of finger flame acceleration in pipes and compared to the case of constant $S_{L}$. Both the 2D channels and cylindrical tubes are considered. The conditions promoting or moderating flame acceleration are identified, and the revisited formulae for the flame shape, velocity and acceleration rate are obtained for different $S_{L}$ - distributions [29].

\section{(iii) Development of a Computational Platform for a Non-uniform Distribution of}

Combustible Coal Dust Particles - (Paper 4) - In this section, a particular example of spatial variation of laminar flame speed $S_{L}$ has been considered. Specifically, the propagation of a gaseous-dusty premixed flame front in a channel, a scenario resembling a methane-air fire in a coal mine, is studied by means of computational simulations. The combustible coal dust particles are incorporated into the solver by means of the Seshadri formulation [30] such that a real gaseous-dusty environment is replaced by an "effective fluid" with locally-modified, dustinduced flow and flame parameters. The originality of this work is in the consideration of various spatial dust concentration distributions, such as the homogenous, linear, cubic and parabolic distributions. Specifically, flame acceleration due to wall friction is analyzed for all these distributions; the similarity and differences in the evolutions of the flame morphology and velocity in each of these cases as well as in the case of purely gaseous combustion are identified [31].

In the conclusion section, all the findings are discussed and some possible future studies are proposed.

Briefly, the original contributions of this thesis include the development of a novel predictive mining scenario, based on the previous works on finger [32] and expanding [33] flames. This idea also helped to generate Refs. $[34,35]$ which were then extended in this work to include further parametric study, the incorporation of 2D planar geometry and the approach of 
compressible flow for a cylindrical geometry into the predictive mining fire scenario. Furthermore, this work developed a theoretical model to investigate the effect of local variations (linear, hyperbolic, parabolic) of the laminar flame speed on the global finger-flame acceleration scenario, and developed a computational platform for uniform (homogenous) and non-uniform (linear, cubic, parabolic) distributions of combustible coal dust particles. 


\section{Predictive Mining Fire Scenario}

Historically around the world, the mining industry has one of the highest injury and fatality rates for employees. While mining accidents are caused by a multitude of reasons, spontaneous methane deflagrations and detonations in the presence of coal dust constitute the most common hazard. Coal dust explosion research goes back to the 1800 s, and one of the earliest known studies was carried out by Michael Faraday and Charles Lyell in 1845 [36]. They investigated possible causes of the Haswell coal mine explosion in 1844 and concluded that coal dust particles had a significant effect and contributed to the intensity of the disaster. Among more recent unfortunate examples of such dust/gas disasters, it can mention in passing the 2014 mining catastrophe in Soma, Turkey, which resulted in over 300 deaths. To reduce the risk of these accidental burning events, researchers worldwide, analyze - experimentally, theoretically and computationally - numerous factors affecting the mechanisms of flame propagation and acceleration in methane/air and methane/air/coal-dust mixtures.

In particular, Chatrathi et al. [37] investigated methane/air flame propagation in industrialscale piping. Silvestrini et al. [38] have provided simplified formulas to evaluate the flame velocities as well as the run-up distances of the deflagration-to-detonation transition (DDT) for flammable mixtures in smooth and obstructed tubes. Chen et al. [39] studied the structure and dynamics of flames at various equivalence ratios; they suggested that flame acceleration occurs only after a transition to turbulence, which actually determines the structure of the flame front. In another study, Bi et al. [40] investigated premixed methane/air flames in relatively long pipes by means of numerical simulations. As for burning in a gaseous environment with combustible dust impurities, Kjaldman [41] performed one of the pioneering numerical studies on the subject, employing a computational fluid dynamics (CFD) tool to small-scale dust flames. Furthermore, Liu et al. [42] conducted experiments on coal-dust/air mixture explosions under a weak ignition condition in a horizontal tube with a diameter $\sim 0.2 \mathrm{~m}$. Skjold et al. [43] investigated, experimentally and numerically, constant-volume dusty/gaseous detonations. Houim et al. [44] simulated the interaction of shock waves and the resulting shear layers with the coal-dust layers. Gardner et al. [45] undertook the large-scale experiments to investigate the detonation spreading in flowing coal-dust - air suspensions in a duct with a diameter $0.6 \mathrm{~m}$. Bartknecht $[46,47]$ 
performed experiments on coal-dust - air detonations in two tubes of different diameters and lengths; the maximum flame speeds attained in a tube with a length $130 \mathrm{~m}$ and diameter $2.5 \mathrm{~m}$ were $500 \mathrm{~m} / \mathrm{s}$ and $700 \mathrm{~m} / \mathrm{s}$, the dust concentrations of $250 \mathrm{~g} / \mathrm{m}^{3}$ and $500 \mathrm{~g} / \mathrm{m}^{3}$, respectively. In the most recent experiments of Ajrash et al. [48-50] the impacts of coal dust on the methane deflagration and explosion characteristics are investigated in a large-scale cylindrical pipe $(0.5$ meter). The effects of methane concentrations (in the range of 1.25-9.5\%) and coal dust concentrations (in the range of $0-30 \mathrm{~g} / \mathrm{m}^{3}$ ) on the pressure rise, flame intensity, ignition source energy as well as pressure and flame velocities are identified.

Nevertheless, up to now there has not been a unified analysis which capturs all the fundamentals and provids enough detail about a flame acceleration mechanism, starting from the initial stages of the process to the onset of the detonation. The present section of the thesis is a step in this direction.

Earlier experiments by Oppenheim [51] and Urtiew [52] were devoted to hydrogen - oxygen flame acceleration, at the initial stage of burning, and transition to detonation. It was concluded that an initially laminar accelerating flame creates compression and then shock waves ahead of it, which subsequently leads to flame turbulization and the detonation onset. Clanet and Searby [53] have identified the "finger-shaped" mechanism of flame acceleration at the early stages of burning in tubes, which has been subsequently justified numerically and developed into a quantitative theory [32]. Specifically, a flame propagating in a cylindrical tube with idealized slip adiabatic walls was considered, with one end of the tube being closed, and a flame being ignited at the symmetry axis at the closed end, and propagating to the open one. In that case, the flame front develops from a hemispherical shape at the beginning to a "finger"-shape (see Fig. 2.1), accompanied by the concomitant exponential growth of surface area of the flame front and, thereby, an associated increase in the flame velocity. This acceleration is fast, but lasts only for a short time interval: until a flame skirt contacts a wall. This acceleration mechanism is Reynoldsindependent, and is therefore equally strong in micro-tubes and mining passages. However, in the practical reality, the Re-dependent agents such as combustion instabilities and/or turbulence provide the corrections to the flame acceleration scenario, making it actually Reynoldsdependent as well [54]. 


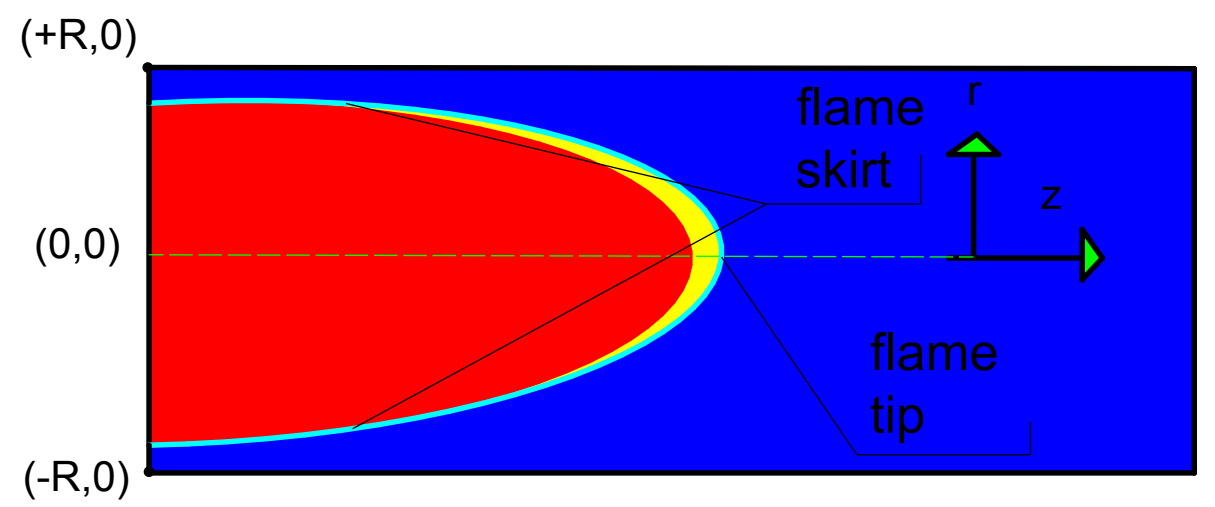

Figure 2.1: 'Finger' model of flame propagation.

Similar to any premixed flame, one of the key flame characteristics in the present problem is the unstretched laminar flame velocity, $S_{L}$, which is a function of thermal-chemical properties of the fuel mixture, in particular, the fuel-to-oxidizer equivalence ratio $\phi$. For the methane/coaldust flames, the laminar flame velocity also depends on the coal-dust parameters such as the size and concentration of the dust particles. Table 2.1 summarizes the typical factors causing flame corrugation/acceleration. In this section, some of them are employed to quantify the mining flame scenario and fix a relevance of any particular mechanism to the mining passage geometry.

Table 2.1: Various mechanisms of flame corrugation/acceleration in tunnels/channels [27].

\begin{tabular}{l|lll}
\hline $\begin{array}{l}\text { Factors causing flame } \\
\text { corrugation/acceleration }\end{array}$ & Re-dependence & $\begin{array}{l}\text { Relevance to } \\
\text { mines }\end{array}$ & $\begin{array}{l}\text { Relevance to } \\
\text { micro-scales }\end{array}$ \\
\hline $\begin{array}{l}\text { Flame instability } \\
\text { Finger flame }\end{array}$ & YES, $\uparrow$ Re & YES & NO \\
Wall friction & NO & YES & YES \\
$\begin{array}{l}\text { Obstacles } \\
\text { Turbulence }\end{array}$ & YES, $\downarrow$ Re & NO & YES \\
\hline
\end{tabular}

First, an initially smooth flame front is subjected to the cellular Darrieus-Landau $(D L)$ instability [5]. While this effect is negligible in micro/meso-scales, it gets stronger with the increase in size, providing a 2.5-10 increase in the flame velocity in tunnels of a human height size (say, 1.7-2 m diameter) $[55,56]$. Second, when a flame front starts approaching the tunnel sidewall, it acquires a "finger-like" shape $[32,53,57]$. Then the flame surface area grows quite fast, promoting the flame velocity by one more order of magnitude by the time the flame skirt 
contacts the wall [32]. Thereafter, this acceleration stops. In the present dissertation, the first mechanism is combined with the second one, for the first time, in order to predict and quantify the timing and locus of these stages in a coal mine. As a result, within the laminar approach, a quantitative predictive scenario of a premixed methane-air burning accident in a mining passage - from the earlier stage of flame acceleration until the transition to a detonation - is provided.

Other potential factors that may influence the process include the effects of wall friction [17] and obstacles [19], as well as post-finger tulip flame formation and turbulence [58], leading to a flame-shock interaction until a detonation onset [59]. However, these factors require extensive research, thereby beyond the scope of the present dissertation but will be proposed as a possible future study. In future studies, the role of turbulence may potentially be incorporated into the present formulation, as an option, by replacing the laminar flame velocity $S_{L}$ with a local turbulent flame velocity $S_{T}$, with the ratio $S_{T} / S_{L}$ obtained analytically, or computationally from a relevant turbulent flame speed model, or phenomenologically from an experiment. As for flame acceleration due to wall friction [17], it weakens significantly with the Reynolds number, being thereby minor in a coal mine. In contrast, a "tooth-brush" array of in-built obstacles generates extremely fast flame acceleration, and this mechanism is Re-independent too, which makes it potentially relevant to the coalmining geometry if obstacles are large enough [19]. However, the role of obstacles in the mining accidents requires separate investigation, which should be undertaken elsewhere.

Overall, the primary focus of the present section is the development of a predictive and quantitative scenario of a mining accident, aiming to provide guidance for preventing and mitigating the disasters associated with gaseous deflagrations and detonations in coal mines. The analytical formulation is presented in Sec. 2.1 in the limit of incompressible flow properties, while Sec. 2.2 is devoted to the parametric study. In Sec. 2.3, the effect of dust particles is considered. The impact of gas compression is taken into account in Sec. 2.4 and the results are discussed in Sec. 2.5.

Specifically, the key characteristics have been identified for different stages of the process and the timing and the burning rate for each stage have been predicted. The input parameters for the formulation include the equivalence ratio, the transport properties of the methane/air/coal- 
dust mixture, as well as the size and concentration of the coal-dust particles (with the laminar flame velocity $S_{L}$ and the thermal expansion coefficient $\Theta$ being coupled to them). Another set of parameters is coupled to the size and configuration of a mining passage.

\subsection{Analytical Formulation (Incompressible Flow)}

Initially, a low Mach-number combustion model [32] is employed, which is relatively simple and provides reasonable evaluations even in the case of compressible flows; see a justification in Ref. [57] for more details (a rigorous extension of this formulation accounting for the compressibility effects will be presented in the Sec. 2.4).

Specifically, here an accidental ignition of a methane-air or propane-air mixture that occurred at a distance $H$ from a tunnel sidewall is considered as illustrated in Figure 2.2(a). An embryonic flame propagates outwardly from the ignition point. It is initially spherically-smooth as any tendency to excite a combustion instability is suppressed by the stretch-effect induced by an expanding flame. The process is controlled by the interplay between the flame stretch and the mixture (non)equi-diffusion. The critical issue at an early stage of burning is whether an embryonic flame be sustained. In this respect, the flame front evolution depends on the Lewis number $L e$ such that sustained combustion is possible for $L e>1$ mixtures, whereas for $L e<1$ mixters, a flame embryo needs to attain a minimum radius, through the initial spark energy, before sustained propagation is possible $[60,61]$. Consequently, keeping $L e<1$ in a mining environment may improve the flame safety standards. It is also noted that $L e \sim 1$ for the majority of methane - air flames.

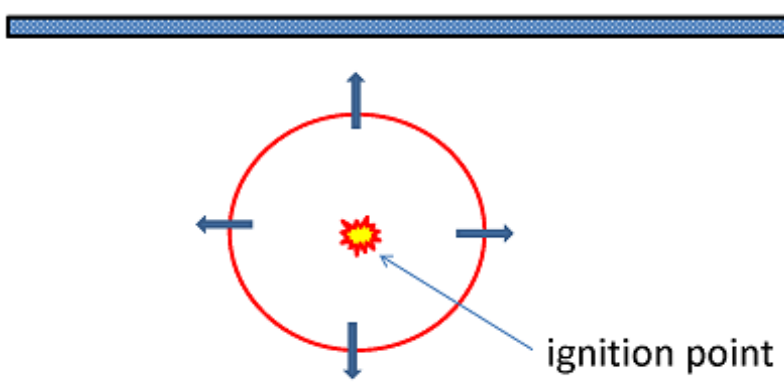

(a)

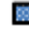

ignition point
Figure. 2.2: Illustration of quasi-spherical flame expansion: the stages of ignition, uniform propagation of a smooth front (a) as well as self-similar acceleration of a cellular front (b). 


\section{Stage 1: Quasi-spherical, self-similar accelerative flame expansion}

Let us consider the case when a flame has survived and keeps propagating. The present dissertation focuses mostly on large scales such that the flame stretch will later be neglected. At the early stage of burning, the flame front expands with a constant speed, $d R_{f} / d t=\Theta S_{L}$, with respect to the ignition point, where $R_{f}(t)$ is the instantaneous flame radius, and the thermal expansion factor $\Theta$ is coupled to the equivalence ratio $\phi$. As a flame "ball" grows in size and the stretch intensity reduces, the diffusional-thermal cells would develop over the surface of Le $<1$ flames [2]. Subsequently, the flame thickness relative to the global flame radius is reduced, leading to the onset of the hydrodynamic $(D L)$ flame instability mode. The latter generates hydrodynamic cells over the flame surface, regardless of $L e$, and will eventually dominate in the surface morphology $[2,25]$. The continuous generation of new cells leads to the continuous increase in the flame surface density and thereby an expanding flame self-accelerates in a scale-invariant (self-similar) manner as illustrated in Fig. 2.2b. According to numerous experimental and computational studies, a reasonable fitting law for such acceleration is [33]

$$
R_{f}=R_{0}+C t^{n} \approx C t^{n},
$$

where $R_{0}$ plays the role of a critical radius related to the transition to the cellular flame structure (it can be neglected within the frame of a large-scale formulation), $n \approx 1.3-1.4$ in the majority of studies; and the factor $C$ can be evaluated as $[16,33]$

$$
C=k_{D L}^{n-1}\left(\Theta S_{L} / n\right)^{n},
$$

where $k_{D L}$ is the DL cutoff wavenumber that appears in the Pelce-Clavin dispersion relation [24], see Eq. (1.6), and it is coupled to the DL critical wavelength as $k_{D L}=2 \pi / \lambda_{D L}$. In the present work, the following formulas for $\lambda_{D L}$ and, respectively, $k_{D L}$ are employed [58]

$$
\lambda_{D L}=2 \pi L_{f}\left(1+\frac{(\Theta+1)}{(\Theta-1)^{2}} \Theta \ln \Theta\right), \quad k_{D L}=L_{f}^{-1}\left(1+\frac{(\Theta+1)}{(\Theta-1)^{2}} \Theta \ln \Theta\right)^{-1} .
$$

With power-law flame acceleration, Eq. (2.1), the global (radial) flame velocity with respect to the ignition point is no longer constant $\Theta S_{L}$, but a time-dependent quantity

$$
d R_{f} / d t=n C t^{n-1}=\left(k_{D L} / n\right)^{n-1}\left(\Theta S_{L}\right)^{n} t^{n-1} .
$$


To evaluate the instantaneous global flame velocity with respect to the fuel mixture, the result (2.4) is divided by $\Theta$, namely

$$
U_{D L}=\frac{1}{\Theta} \frac{d R_{f}}{d t}=\frac{n C}{\Theta} t^{n-1}=S_{L}^{n}\left(\frac{\Theta}{n} k_{D L}\right)^{n-1} t^{n-1} .
$$

\section{Stage 2: Finger-like flame acceleration}

Generally speaking, Eqs. (2.1) - (2.5) describe the accelerative flame expansion in an opening. In practice, as soon as a flame front approaches (even before contacting) the tunnel/channel wall, the difference between the radial and the axial flow velocities modifies the flame shape, forming two outwardly propagating "finger-like" fronts, as illustrated in Fig. 2.3. In fact, the expansion of the burning matter leads to a strong flow in the axial direction, which drifts the tip of a "finger"shaped flame. Due to the elongated shape, the surface area of such a flame front is much larger than the passage cross section, which causes the flame to accelerate. However, this acceleration stops when a flame "skirt" contacts a passage wall. By the end of this process, the distance from the ignition point to the flame tip is much larger than the passage radius.

Next, the analysis of an expanding flame is combined with a finger-flame formulation [32] such that a time-dependent quantity $U_{D L}$, Eq. (2.5), is incorporated instead of the constant $S_{L}$. The rational for such a consideration is the scale- and time-separations between the formulations: the tunnel width is much larger than the $D L$ cells, and the finger-flame acceleration is much stronger than that associated with the $D L$ instability. To generalize the approach, both the twodimensional (2D) planar and cylindrical geometries are considered and compared. In fact, a real coal mine tunnel may have a rectangular/square cross-section, which is actually neither $2 \mathrm{D}$ nor cylindrical, but in between these two geometries. Sections 2.1.1 and 2.1.2, present the analytical formulations for the 2D-planar and cylindrical-axisymmetric configurations, respectively. 


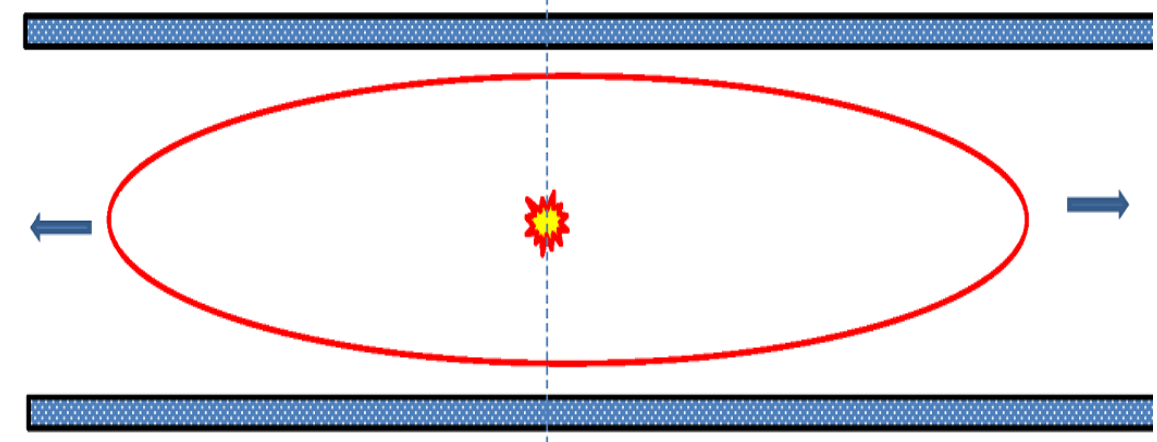

Figure. 2.3: Illustration of finger-like flame acceleration.

(a)

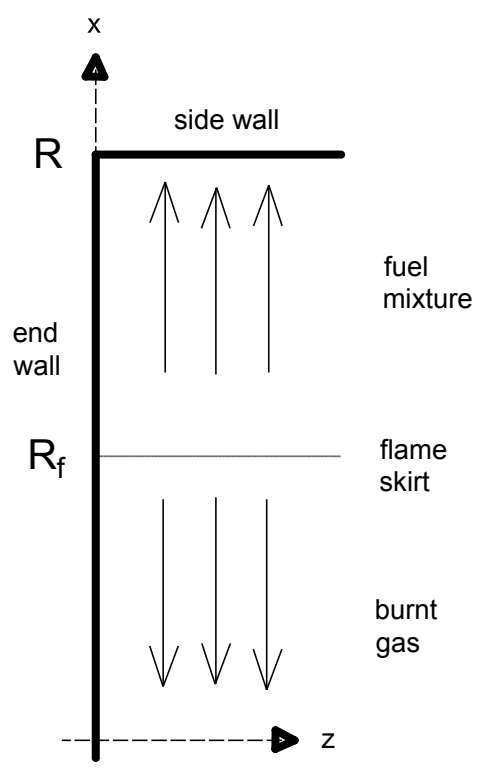

(b)

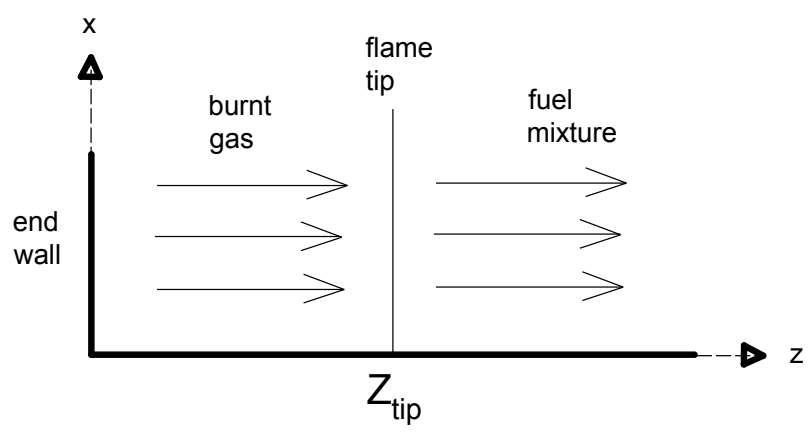

Figure 2.4: A flow close to a tunnel end-wall (a) and axis (b).

\subsubsection{D Planar Geometry}

The sketch of the coordinate system for the flow near the end-wall and the centerline of the tunnel is shown in Figure 2.4. First a 2D geometry is considered. Then the incompressible continuity equation reads

$$
\frac{\partial u_{x}}{\partial x}+\frac{\partial u_{z}}{\partial z}=0
$$

The boundary conditions are $\left.u_{z}\right|_{z=0}=0,\left.u_{x}\right|_{x=R_{f}}=0$. By assuming a potential flow, the axial and radial flow velocities ahead of (subscript " 1 ") and behind (subscript " 2 ") the flame front read 


$$
u_{z, 1}=A_{1}(t) z, \quad u_{x, 1}=A_{1}(t)(H-x), \quad u_{z, 2}=A_{2}(t) z, \quad u_{x, 2}=-A_{2}(t) x,
$$

where the factors $A_{1}$ and $A_{2}$ depend on time only. While the flow is generally rotational in the burnt matter, due to a curved shape of the flame front, the flow can be treated as potential close to the end-wall, where a flame front is locally planar. The matching conditions at a flame front, $x=R_{f}$, are

$$
\frac{d R_{f}}{d t}-u_{x, 1}=U_{D L}(t), \quad u_{x, 1}-u_{x, 2}=(\Theta-1) U_{D L}(t), \quad u_{z, 1}=u_{z, 2},
$$

where the first equation specifies the flame propagation velocity with respect to the fuel mixture, the second describes the jump of the normal velocity, and the third describes the continuity of the tangential velocity. Substituting Eq. (2.7) into Eq. (2.8) one finds $A_{1}(t)=A_{2}(t)=(\Theta-1) U_{D L}(t) / H$ and then the evolution equation for the flame skirt reads:

$$
\frac{d R_{f}}{d t}=\left\{(\Theta-1)\left(1-\frac{R_{f}}{H}\right)+1\right\} U_{D L}=\left\{(\Theta-1)\left(1-\frac{R_{f}}{H}\right)+1\right\} S_{L}^{n}\left(\frac{\Theta}{n} k_{D L}\right)^{n-1} t^{n-1},
$$

with the initial condition $R_{f} \mathrm{l}_{t=0}=0$, and the solution:

$$
\begin{aligned}
& t\left(R_{f}\right)=\left\{\frac{\Theta H}{(\Theta-1) C} \ln \left(\frac{\Theta H}{\Theta H-(\Theta-1) R_{f}}\right)\right\}^{1 / n}=\frac{n}{\Theta S_{L}}\left\{\frac{\Theta H}{(\Theta-1) k_{D L}^{n-1}} \ln \left(\frac{\Theta H}{\Theta H-(\Theta-1) R_{f}}\right)\right\}^{1 / n}, \\
& \frac{R_{f}(t)}{H}=\frac{\Theta}{\Theta-1}\left\{1-\exp \left[-\frac{\Theta-1}{\Theta H} C t^{n}\right]\right\}=\frac{\Theta}{\Theta-1}\left\{1-\exp \left[-\frac{\Theta-1}{\Theta H}\left(k_{D L}^{n-1}\left(\frac{\Theta S_{L}}{n}\right)^{n}\right) t^{n}\right]\right\} .
\end{aligned}
$$

The characteristic time instant devoted to the transition from a globally-spherical to a fingerlike flame shape, $t_{s p h}$, and the associated flame skirt location, $R_{f}\left(t_{s p h}\right)$, can be evaluated as

$$
t_{s p h} \approx\left(\frac{\Theta H}{(\Theta-1) C}\right)^{1 / n}=\frac{n}{\Theta S_{L}}\left(\frac{\Theta H}{(\Theta-1) k_{D L}^{n-1}}\right)^{1 / n}, \quad R_{f}\left(t_{s p h}\right)=\frac{\left(1-e^{-1}\right) \Theta H}{(\Theta-1)} \approx 0.632 \frac{\Theta H}{(\Theta-1)},
$$

with the burning rate at this instant being

$$
U_{D L}\left(t_{s p h}\right)=\frac{n C}{\Theta} t_{s p h}^{n-1}=n\left(\frac{C}{\Theta}\right)^{1 / n}\left(\frac{H}{\Theta-1}\right) \frac{n-1}{n} .
$$

The next focus is on the flame tip, which evolution equation reads

$$
\frac{d Z_{t i p}}{d t}=(\Theta-1) U_{D L}(t) \frac{Z_{t i p}}{H}+\Theta U_{D L}(t)
$$


with the initial condition $\left.Z_{t i p}\right|_{t=0}=0$, and the solution:

$$
Z_{t i p}=\frac{\Theta H}{(\Theta-1)}\left\{\exp \left[\frac{(\Theta-1)}{\Theta} \frac{C t^{n}}{H}\right]-1\right\}=\frac{\Theta H}{(\Theta-1)}\left\{\exp \left[\frac{(\Theta-1)}{\Theta} \frac{k_{D L}^{n-1}}{H}\left(\frac{\Theta S_{L}}{n}\right)^{n} t^{n}\right]-1\right\} .
$$

The flame skirt (in fact, its first "wing") contacts the tunnel side wall when $R_{f}=H$, i.e.:

$$
t_{\text {wall.1 }}=\left\{\frac{\Theta H}{(\Theta-1) C} \ln (\Theta)\right\}^{1 / n}=\frac{n}{\Theta S_{L}}\left\{\frac{\Theta H}{(\Theta-1) k_{D L}^{n-1}} \ln (\Theta)\right\}^{1 / n} .
$$

The second flame wing contacts the opposite wall a little later, when $R_{f}=2 R-H$,

$$
t_{\text {wall.2 }}=\left\{\frac{\Theta(2 R-H)}{(\Theta-1) C} \ln (\Theta)\right\}^{1 / n}=\frac{n}{\Theta S_{L}}\left\{\frac{\Theta(2 R-H)}{(\Theta-1) k_{D L}^{n-1}} \ln (\Theta)\right\}^{1 / n} .
$$

Obviously, $t_{\text {wall }, 1}=t_{\text {wall }, 2}$ if $H=R$ and $t_{\text {wall }} / t_{s p h}=\sqrt[n]{\ln \Theta}$. The velocity of the flame tip in the laboratory reference frame and its acceleration are respectively given by:

$$
\begin{aligned}
& \frac{d Z_{t i p}}{d t}=U_{t i p}=n C t^{n-1} \exp \left(\frac{(\Theta-1) C t^{n}}{\Theta H}\right), \\
& \frac{d^{2} Z_{t i p}}{d t^{2}}=a_{t i p}=n C t^{n-1} \exp \left(\frac{(\Theta-1) C t^{n}}{\Theta H}\right)\left\{(n-1) t^{-1}+n t^{n-1} \frac{\Theta-1}{\Theta} \frac{C}{H}\right\} .
\end{aligned}
$$

One can also readily check that in the case of $n=1$, the $D L$ instability disappears and all these formulas reproduce their counterparts of Ref. [57].

\subsubsection{Cylindrical Axisymmetric Geometry}

Here, a similar analytical formulation is developed for the cylindrical-axisymmetric geometry. In this case, the continuity equation for the incompressible flow reads

$$
\frac{1}{r} \frac{\partial\left(r u_{r}\right)}{\partial r}+\frac{\partial u_{z}}{\partial z}=0
$$

with the boundary conditions $\left.u_{z}\right|_{z=0}=0,\left.u_{r}\right|_{r=r_{f}}=0$. Similar to the $2 \mathrm{D}$ case, assuming potential flow in the fuel mixture, velocity components (with singularity occurring) can be found as

$$
u_{z, 1}=A_{1}(t) z, \quad u_{r, 1}=\frac{A_{1}(t)}{2}\left(\frac{H^{2}}{r}-r\right), \quad u_{z, 2}=A_{2}(t) z, \quad u_{r, 2}=-\frac{A_{2}(t)}{2} r .
$$


The matching conditions are given by Eq. (2.8). Then $A_{1}(t)=A_{2}(t)=2(\Theta-1) U_{D L}(t) R_{f} / H^{2}$. Altogether, Eqs (2.6), (2.20) - (2.21) provide the evolution equation for the flame skirt:

$$
\frac{d R_{f}}{d t}=\left\{\Theta-(\Theta-1) \frac{R_{f}^{2}}{H^{2}}\right\} U_{D L}=\left\{\Theta-(\Theta-1) \frac{R_{f}^{2}}{H^{2}}\right\} S_{L}^{n}\left(\frac{\Theta}{n} k_{D L}\right)^{n-1} t^{n-1},
$$

with the solution

$$
\begin{aligned}
& t\left(R_{f}\right)=\left\{\frac{\Theta H}{2 \alpha C} \ln \left(\frac{\Theta+\alpha\left(R_{f} / H\right)}{\Theta-\alpha\left(R_{f} / H\right)}\right)\right\}^{1 / n}=\frac{n}{\Theta S_{L}}\left\{\frac{\Theta H}{2 \alpha k_{D L}^{n-1}} \ln \left(\frac{\Theta+\alpha\left(R_{f} / H\right)}{\Theta-\alpha\left(R_{f} / H\right)}\right)\right\}^{1 / n}, \\
& \frac{R_{f}(t)}{H}=\frac{\Theta}{\alpha} \tanh \left(\frac{\alpha}{\Theta} \frac{C t^{n}}{H}\right)=\frac{\Theta}{\alpha} \tanh \left(\frac{\alpha}{\Theta H} k_{D L}^{n-1}\left(\frac{\Theta S_{L}}{n}\right)^{n} t^{n}\right),
\end{aligned}
$$

where $\alpha=\sqrt{\Theta(\Theta-1)}$. The characteristic time devoted to the transition from a globallyspherical to a finger-like flame shape, $t_{s p h}$, and the flame skirt location at this instant, $R_{f}\left(t_{s p h}\right)$, can be evaluated as:

$$
t_{s p h} \approx\left(\frac{\Theta H}{2 \alpha C}\right)^{1 / n}=\frac{n}{\Theta S_{L}}\left(\frac{\Theta H}{2 \alpha k_{D L}^{n-1}}\right)^{1 / n}, \quad R_{f}\left(t_{s p h}\right)=\frac{\Theta H}{\alpha} \tanh (0.5) \approx 0.46 \sqrt{\frac{\Theta}{\Theta-1}} H,
$$

with the associated corrugated flame velocity being

$$
U_{D L}\left(t_{s p h}\right)=\frac{n C}{\Theta} t_{s p h}^{n-1}=n\left(\frac{C}{\Theta}\right)^{1 / n}\left(\frac{H}{2 \alpha}\right)^{(n-1) / n} .
$$

Next, the flame tip, which evolution equation in this geometry reads

$$
\frac{d Z_{t i p}}{d t}=2(\Theta-1) U_{D L}(t) \frac{R_{f}(t) Z_{t i p}}{H^{2}}+\Theta U_{D L}(t),
$$

with the solution

$$
Z_{t i p}=\frac{\Theta H}{2 \alpha} \sinh \left(2 \frac{\alpha}{\Theta} \frac{C t^{n}}{H}\right)=\frac{H}{2} \sqrt{\frac{\Theta}{\Theta-1}} \sinh \left(2 \sqrt{\frac{\Theta-1}{\Theta}} \frac{k_{D L}^{n-1}}{H}\left(\frac{\Theta S_{L}}{n}\right)^{n} t^{n}\right) .
$$

The first wing of the flame skirt contacts the tunnel side wall when $R_{f}=H$, i.e.

$$
t_{\text {wall }, 1}=\left\{\frac{\Theta H}{2 \alpha C} \ln \left(\frac{\Theta+\alpha}{\Theta-\alpha}\right)\right\}^{1 / n}=\frac{n}{\Theta S_{L}}\left\{\frac{\Theta H}{2 \alpha k_{D L}^{n-1}} \ln \left(\frac{\Theta+\alpha}{\Theta-\alpha}\right)\right\}^{1 / n} .
$$

The second flame wing contacts the opposite wall at 


$$
t_{\text {wall }, 2}=\left\{\frac{\Theta(2 R-H)}{2 \alpha C} \ln \left(\frac{\Theta+\alpha}{\Theta-\alpha}\right)\right\}^{1 / n}=\frac{n}{\Theta S_{L}}\left\{\frac{\Theta(2 R-H)}{2 \alpha k_{D L}^{n-1}} \ln \left(\frac{\Theta+\alpha}{\Theta-\alpha}\right)\right\}^{1 / n},
$$

when $R_{f}=2 R-H$, Obviously, $t_{\text {wall }, 1}=t_{\text {wall }, 2}$ if $H=R$ and $t_{\text {wall }} / t_{\text {sph }}=\sqrt[n]{\ln [(\Theta+\alpha) /(\Theta-\alpha)]}$. The flame tip velocity and acceleration in the laboratory reference frame read

$$
\begin{aligned}
& \frac{d Z_{\text {tip }}}{d t}=U_{t i p}=n C t^{n-1} \cosh \left(\frac{2 \alpha C t^{n}}{\Theta H}\right), \\
& \frac{d^{2} z_{t i p}}{d t^{2}}=a_{t i p}=n C t^{n-2}\left\{\frac{2 \alpha n C t^{n}}{\Theta H} \sinh \left(\frac{2 \alpha n C t^{n}}{\Theta H}\right)+(n-1) \cosh \left(\frac{2 \alpha n C t^{n}}{\Theta H}\right)\right\} .
\end{aligned}
$$

Again, in the case of $n=1$, the $D L$ instability disappears and all these formulas reproduce their counterparts of Ref. [57].

\subsection{Results and Discussion (Incompressible Flow)}

In this section, the analytical results of Sec. 2.1 are thoroughly investigated for a set of input parameters. Initially, the gaseous methane- or propane-air flames, of various equivalence ratios, are considered. Subsequently, the analysis is extended to methane/air/coal-dust combustion.

While it is a methane/air accidental explosion that is most relevant to coal mines, for comparison, and to identify the role of the type of a combustible, here potential propane/air flame spreading is also investigated. The thermal expansion factor $\Theta$ and the laminar flame velocity $S_{L}$ are tabulated versus the equivalence ratio $\phi$ in Table 2.2 , for the methane/air mixture, and in Table 2.3, for the propane/air one, respectively [62]. Based on these tables, the characteristic timings of the process, $t_{s p h}$, Eqs. (2.12) and (2.25), and $t_{\text {wall }}$, Eqs (2.16), (2.17) and (2.29), (2.30) are presented in Figs. 2.5 and 2.6 for the 2D planar and cylindrical axisymmetric geometries, respectively, with $n=1.4$ and $R=H=1.05 \mathrm{~m}$ in both figures. It is recalled that

these quantities, $t_{s p h}$ and $t_{\text {wall }}$, play the key roles for flame acceleration and, thereby, the entire flame evolution scenario. Equations (2.9)-(2.11), (2.22)-(2.24) exhibit a globally-spherical (cellular) shape of an expanding flame front while $t<t_{s p h}$. Then the flame skirt slows down, while the flame tip accelerates, thereby leading to very strong elongation and global acceleration 
of the flame front within the interval $t_{\text {sph }}<t<t_{\text {wall }}$. This acceleration is nevertheless limited in time: it terminates as soon as the flame skirt contacts a wall, $t=t_{\text {wall }}$.

Table 2.2: The methane/air flame parameters [62]

\begin{tabular}{llllllllll}
\hline$\phi$ & 0.6 & 0.7 & 0.8 & 0.9 & 1 & 1.1 & 1.2 & 1.3 & 1.4 \\
$\Theta$ & 5.54 & 6.11 & 6.65 & 7.12 & 7.48 & 7.55 & 7.43 & 7.28 & 7.09 \\
$S_{L}(\mathrm{~m} / \mathrm{s})$ & 0.089 & 0.169 & 0.254 & 0.325 & 0.371 & 0.383 & 0.345 & 0.250 & 0.137 \\
\hline
\end{tabular}

Table 2.3: The propane/air flame parameters [62]

\begin{tabular}{llllllllll}
\hline$\phi$ & 0.63 & 0.7 & 0.8 & 0.9 & 1 & 1.1 & 1.2 & 1.3 & 1.4 \\
$\Theta$ & 6.04 & 6.56 & 7.15 & 7.66 & 8.02 & 8.08 & 8 & 7.88 & 7.74 \\
$S_{L}(\mathrm{~m} / \mathrm{s})$ & 0.147 & 0.217 & 0.303 & 0.374 & 0.418 & 0.429 & 0.399 & 0.322 & 0.226 \\
\hline
\end{tabular}

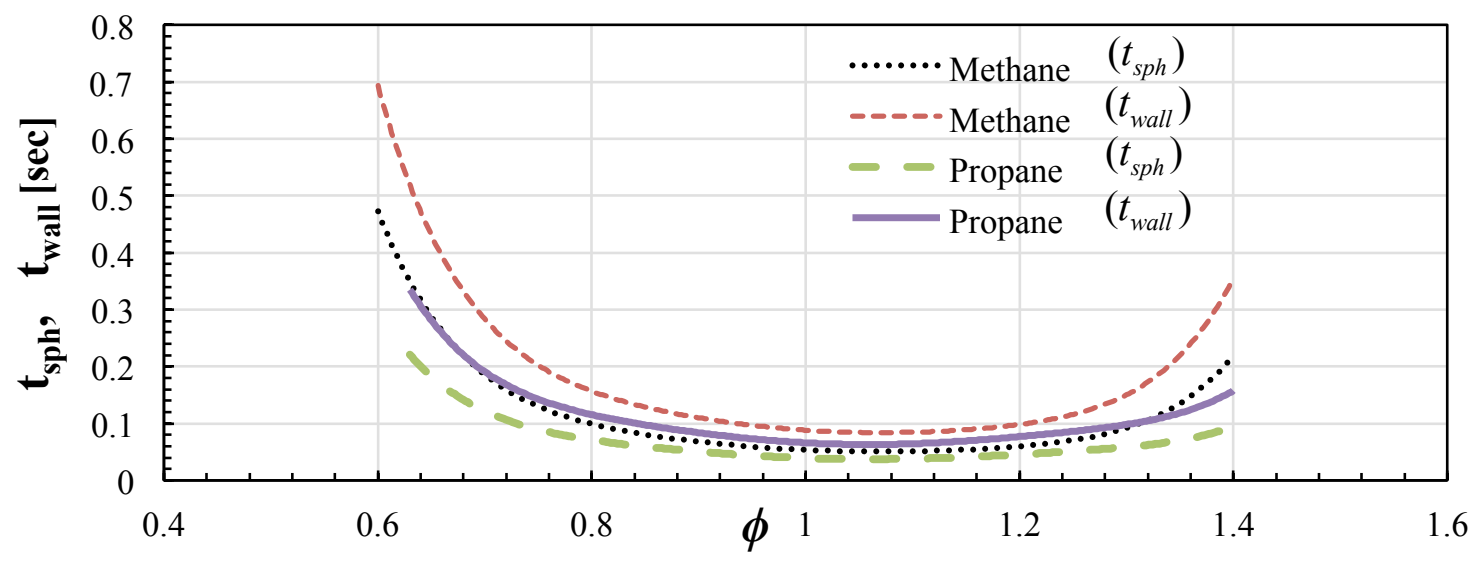

Figure 2.5: 2 planar geometry: The time limitations of finger flame acceleration, $t_{s p h}$, Eq. (2.12), and $t_{\text {wall }}$, Eq. (2.16), versus the equivalence ratio $\phi$ for the propane/air and methane/air flames, $R=H=1.05 \mathrm{~m} n=1.4$. 


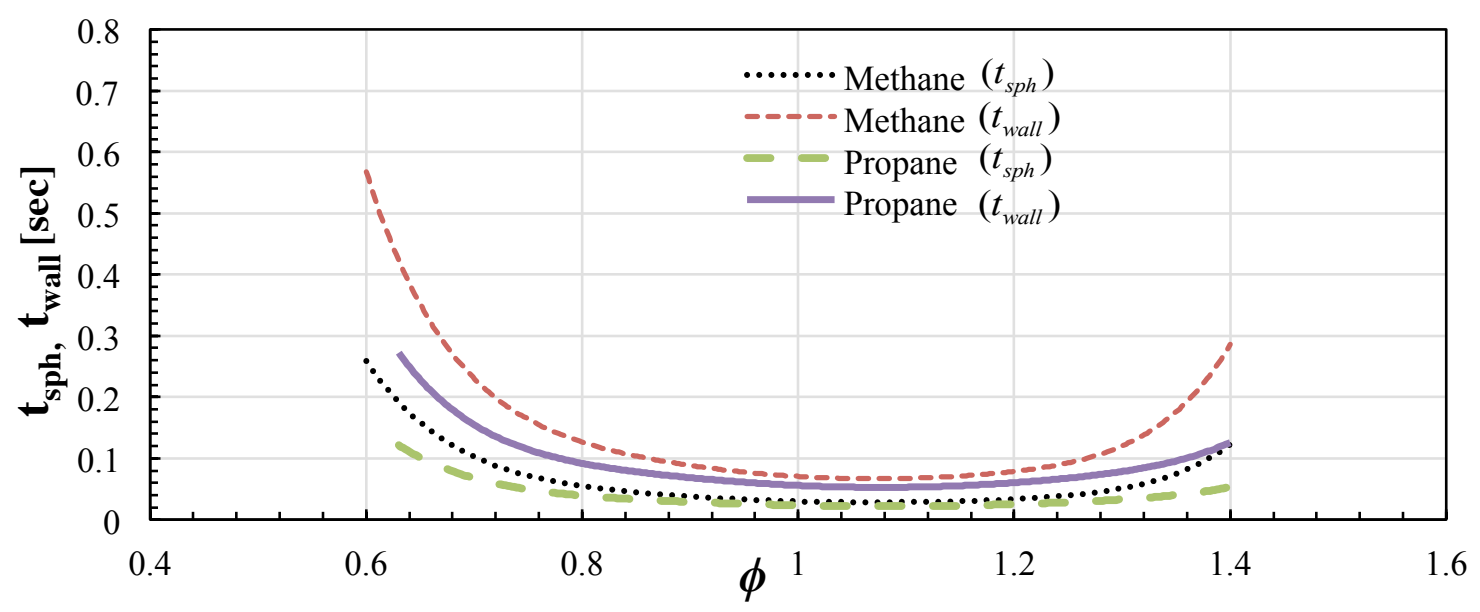

Figure 2.6: Cylindrical axisymmetric geometry: The time limitations of finger flame acceleration, $t_{s p h}$, Eq. (2.25), and $t_{\text {wall }}$, Eq. (2.29), versus the equivalence ratio $\phi$ for the propane/air and methane/air flames, $R=H=1.05 \mathrm{~m} n=1.4$.

The flame tip position, velocity and acceleration are shown in Figs. $2.7-2.9$ for the planar and cylindrical geometries. Here, Figs. 2.7(a) - 2.9(a) demonstrate the time evolution of these quantities, in a stoichiometric mixture, $\phi=1$, while Figs. 2.7(b) $-2.9(\mathrm{~b})$ present the maximal quantitates, attained during finger flame acceleration, versus the equivalence ratio $\phi$, namely: $Z_{\text {tip }} \equiv Z_{\text {tip }}\left(t_{\text {wall }}\right) \equiv \Theta \mathrm{H}$, Eqs. (2.15) and (2.28); $\dot{Z}_{\text {tip }} \equiv U_{\text {tip }}\left(t_{\text {wall }}\right)$, Eqs. (2.18) and (2.31); and $\ddot{Z}_{\text {tip }} \equiv a_{\text {tip }}\left(t_{\text {wall }}\right)$, Eqs. (2.19) and (2.32), respectively. It is seen that a propane/air flame generally spans a greater distance before it contacts a wall, which can be attributed to its higher laminar flame velocity $S_{L}$ at a given equivalence ratio. Besides, a higher flame tip velocity and, thereby, stronger acceleration are attained in the cylindrical configuration as compared to the $2 \mathrm{D}$ one.

As a result, the impacts of the globally-spherical and of the finger-like flame acceleration mechanisms are both significant in a coalmining passage, and they may trigger detonation more effectively in a cylindrical configuration than in a 2D geometry. Specifically, in both cases, the whole process takes less than $0.1 \mathrm{~s}$, during which a flame tip travels around $8 \mathrm{~m}$ (Fig. 2.7). The tip of a methane flame front attains the velocities of $300 \mathrm{~m} / \mathrm{s}$ and $500 \mathrm{~m} / \mathrm{s}$ (in the laboratory reference frame) in the 2D-planar and cylindrical geometries, respectively, Fig. 2.8(a), thus exceeding the nominal value $S_{L}$ by three orders of magnitude, and the laminar flame velocity in the laboratory reference frame, $\Theta S_{L}$, by two orders. 
For propane/air combustion, the burning rate increases even higher, up to $400 \mathrm{~m} / \mathrm{s}$ and 700 $\mathrm{m} / \mathrm{s}$ in the 2D-planar and cylindrical-axisymmetric geometries, respectively. In fact, it is Fig. 2.8 that identifies whether a propagating flame front can attain a sonic/supersonic speed in a coal mine, where a detonation occurs mostly due to an accidental ignition of methane, being thereby one of the major causes for a disaster. For methane/air burning, while such an overcome of the sound barrier is not observed in a $2 \mathrm{D}$ channel, in a cylindrical geometry, it occurs for the equivalence ratios in the range $0.9 \leq \phi \leq 1.2$. For faster, propane/air burning, this range is even wider in the cylindrical geometry, $0.8 \leq \phi \leq 1.3$, and it is also observed in a $2 \mathrm{D}$ planar geometry for $1.0 \leq \phi \leq 1.2$. Consequently, one may expect a deflagration-to-detonation transition (DDT) event to occur in all these cases.
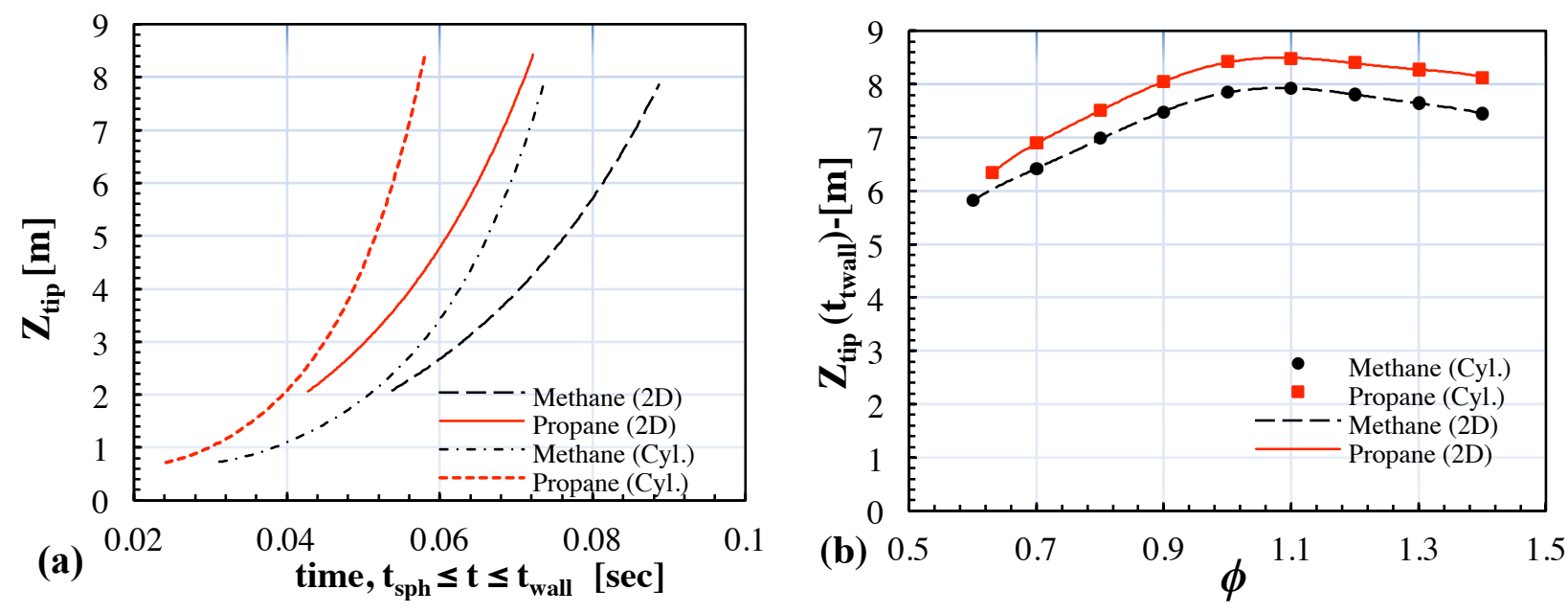

Figure 2.7: (a) Evolution of the flame tip position $Z_{t i p}$ in a stoichiometric $(\phi=1)$ mixture and (b) $Z_{\text {tip }}\left(t_{\text {wall }}\right)$ versus $\phi$ for methane- and propane/air combustion in the 2D planar and cylindrical axisymmetric geometries. 

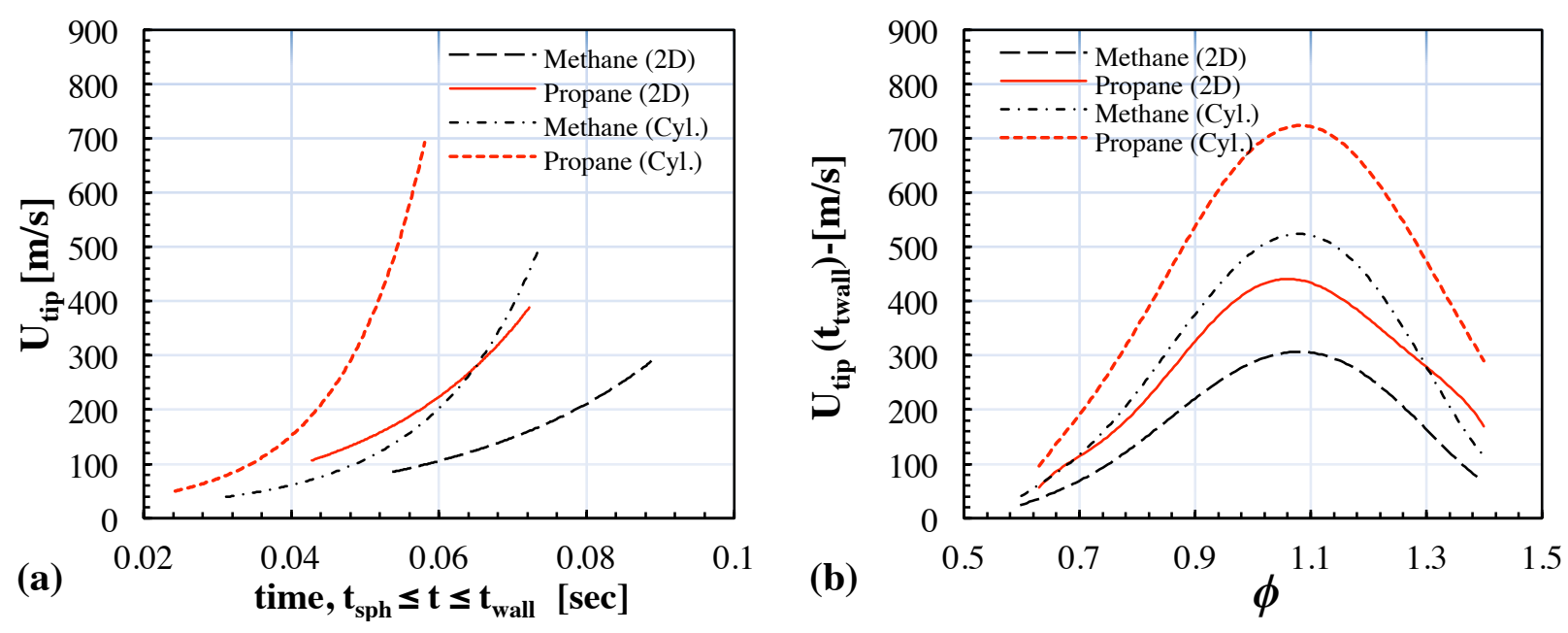

Figure 2.8: (a) Evolution of the flame tip velocity $U_{\text {tip }}$ in a stoichiometric $(\phi=1)$ mixture and (b) $U_{\text {tip }}\left(t_{\text {wall }}\right)$ versus $\phi$ for methane- and propane/air combustion in the 2D-planar and cylindrical-axisymmetric geometries.
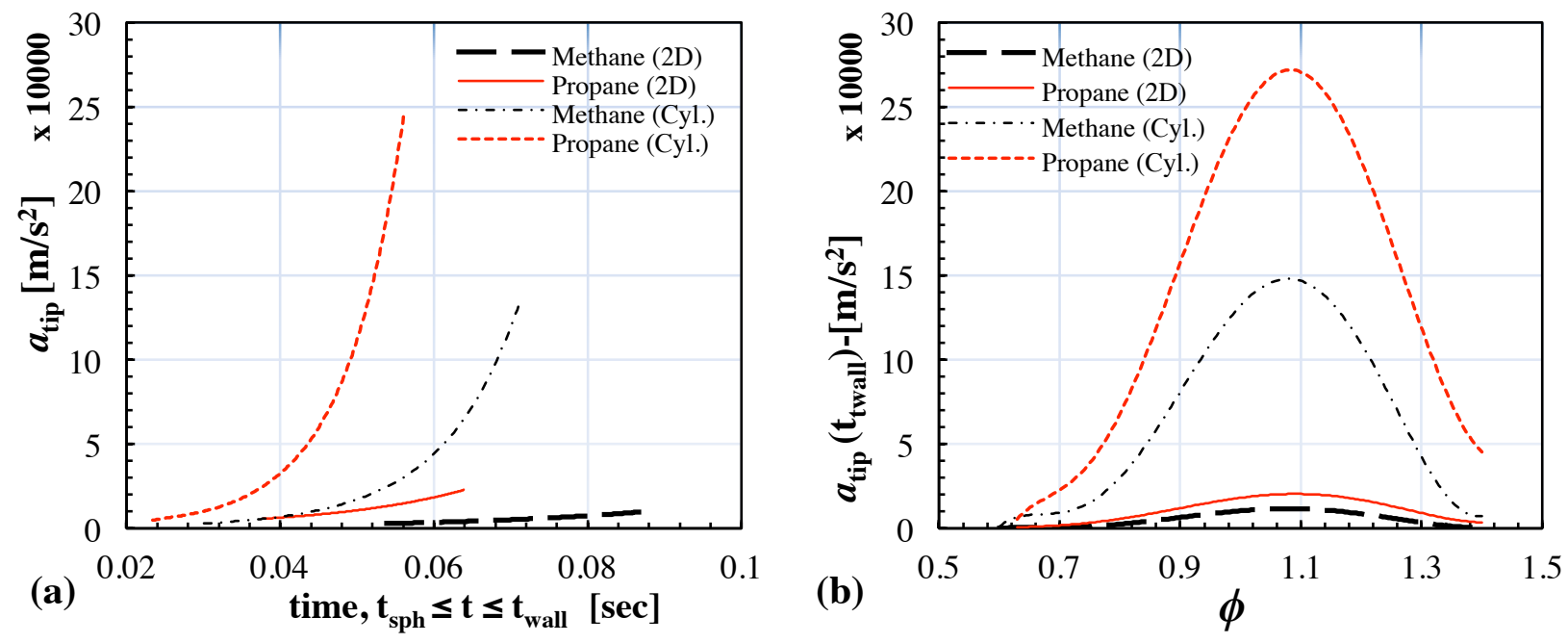

Figure 2.9: (a) Evolution of the flame tip acceleration $a_{t i p}$ in a stoichiometric $(\phi=1)$ mixture and (b) $a_{\text {tip }}\left(t_{\text {wall }}\right)$ versus $\phi$ for methane- and propane/air combustion in the 2D-planar and cylindrical-axisymmetric geometries.

In fact, a reasonable and conventional parameter to analyze flame acceleration as a stage of the DDT process is the so-called run-up distance. Two distinctive definitions for such a quantity are frequently employed in DDT studies: $(i)$ a distance that a flame spans from its ignition to the 
detonation onset; or (ii) a distance, at which the flame velocity in the laboratory reference frame equals the sound speed. In the first case, the run-up distance strongly depends on particular chemical kinetics of the reactions involved. In contrast, in the latter case, the run-up distance is a purely gas-dynamic characteristic of the process. Since the focus of the present dissertation is the gas-dynamics of flame acceleration, the second definition is employed here, namely, the run-up distance is approximated as the flame tip position at the instant when its velocity equals the local sound speed, $d Z_{t i p} / d t=U_{t i p}=c_{o}$. Although it is recognized that such a definition is not accurate, and the detonation does not occur exactly at that instant, still these values correlate, and therefore this is a reasonable approximation. Additionally, one may also define the run-up time as the instant when the flame speed in the laboratory reference frame overcomes the sound barrier. For the 2D-planar geometry, the run-up timing, $t_{r u d}$, can be obtained by equating (2.18) to the sound speed:

$$
c_{0}=\left.\frac{d Z_{\text {tip }}}{d t}\right|_{r . u . d}=\left.U_{\text {tip }}\right|_{r . u . d}=n C t_{r u d}^{n-1} \exp \left(\frac{(\Theta-1) C t_{r u d}^{n}}{\Theta H}\right),
$$

and then, substituting this result into Eq. (2.15), the run-up distance is given by

$$
Z_{\text {rud }}=\frac{\Theta H}{\Theta-1}\left\{\exp \left[\frac{(\Theta-1) C t_{r u d}^{n}}{\Theta H}\right]-1\right\} \text {. }
$$

The cylindrical counterparts of these quantities are obtained in the same manner by using Eqs. (2.31) and (2.28), which yield:

$$
\begin{aligned}
& c_{0}=\left.\frac{d Z_{\text {tip }}}{d t}\right|_{\text {r.u.d }}=\left.U_{\text {tip }}\right|_{r . u . d}=n C t_{r u d}^{n-1} \cosh \left(\frac{2 \alpha C t_{r u d}^{n}}{\Theta H}\right), \\
& Z_{r u d}=\frac{\Theta H}{2 \alpha} \sinh \left(\frac{2 \alpha C t_{r u d}^{n}}{\Theta H}\right) .
\end{aligned}
$$

All these results are shown in Fig. 2.10, which identifies the distance the flame propagates before the detonation onset. 


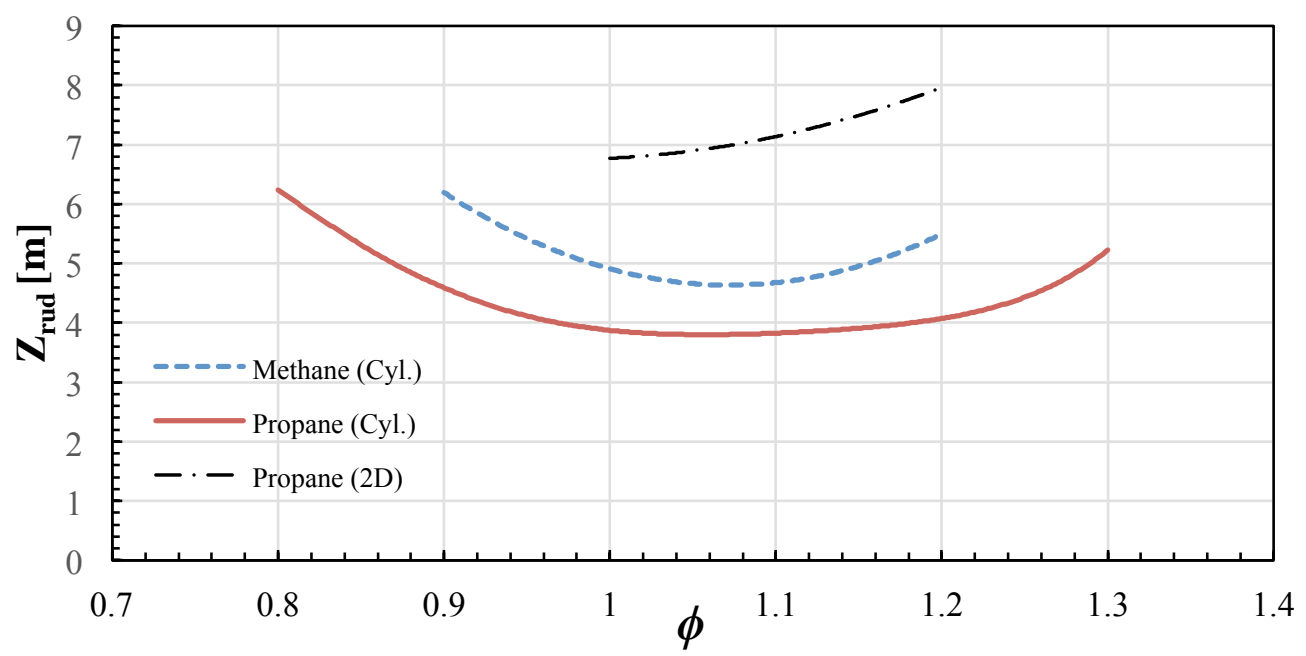

Figure 2.10: The run-up distance, $Z_{r u d}$, versus the equivalence ratio $\phi$ for methane - air flames, in a cylindrical geometry, and for propane - air flames, in both 2D-planar and cylindrical geometries.

\subsection{Extension to Gaseous-Dusty Environment}

One of the major issues in the coal mining industry is that the modern knowledge base about dust explosions does not provide an acceptable level of risk assessments because the physics of the controlling mechanisms, associated with particle/gaseous flames, have not been explored sufficiently [63]. Specifically, modern coalmining machinery has significantly increased the portion of small-size coal dust collected in intake/return airways [64]. Consequently, a model capable of quantifying the probability and associated hazards of spontaneous ignition, deflagration and the likelihood of a deflagration-to-detonation transition (DDT) event is highly needed.

Next, the formulation is extended from combustion of a purely gaseous methane/air mixture to that with coal-dust impurities. For this purpose, the Seshadri formulation [30] is implemented, which expresses the "gaseous-dusty" unstretched laminar flame velocity, $S_{d, L}$, as a function of local thermal-chemical properties of the gas and coal dust in the form

$$
S_{d, L}=\frac{1}{Z e} \sqrt{\frac{2 B k_{u}}{\rho_{u} C_{T}} \exp \left(-\frac{E_{a}}{R_{u} T_{f}}\right)}, \quad Z e=\frac{E_{a}\left(T_{f}-T_{u}\right)}{R_{u} T_{f}^{2}},
$$

where $E_{a}$ is the activation energy, $Z e$ the Zel'dovich number, and 


$$
C_{T}=C_{P}+C_{s} n_{s} \frac{4 \pi r_{s}^{3}}{3} \frac{\rho_{s}}{\rho}
$$

the whole specific heat of the mixture, with $C_{p}$ and $C_{s}$ being that of the gas and coal dust particles, respectively. Here $\rho$ is the density of the mixture, which can be expressed as $\rho=\rho_{u}+c_{s}$, where $\rho_{u}$ is the fresh gas density and $c_{s}$ the concentration of particles. The quantity $n_{s}=\left(c_{s} / \rho_{s}\right) / V_{s}$ is the number of particles per unit volume, with $V_{\mathrm{s}}=4 \pi r_{s}^{3} / 3$ being the volume of a single particle and $r_{s}$ being the radius of this particle. The laminar flame velocity, $S_{d, L}$, can generally increase or decrease in the presence of coal particles. On the one hand, the flame speed is promoted by the effect of volatiles released from the coal particles through the gaseous mixture, which is accounted as an additional fuel source for the combustion process in the reaction zone. As a result, the growth of the equivalence ratio promotes the flame temperature and, thereby, the flame propagation velocity. On the other hand, the coal dust particles gain heat from the flame during the devolatilization process, thereby acting as a heat sink. This reduces the flame temperature and, thereby, the flame velocity [65]. To be specific, Ref. [30] suggested the following expression for the devolatilization rate of the coal particles (the mass of a gaseous fuel vaporized per unit volume per second):

$$
w_{v}^{\prime}=A n_{s} 4 \pi r^{2} T_{s}^{N}
$$

Similar to Ref. [30], in the present dissertation $A=3.4 \cdot 10^{-5} \mathrm{~kg} \mathrm{~m}^{-2} \mathrm{~s}^{-1} \mathrm{~K}, N=1.33$ is used. The temperature of a coal particle is approximated as $T_{s}=\left(T_{v}+T_{b}\right) / 2$, where $T_{v}$ is the devolatilization temperature, which is taken here to be $600 \mathrm{~K}$, and $T_{b}$ is the adiabatic flame temperature based on the purely methane - air equivalence ratio. Among various methods to find $T_{b}$, here it is evaluated as a $5^{\text {th }}$-order polynomial function of the equivalence ratio [66]

$$
\begin{aligned}
& T_{b}=\left(-2.21 \times 10^{4}\right) \phi^{5}+\left(8.042 \times 10^{4}\right) \phi^{4}+\left(-1.171 \times 10^{5}\right) \phi^{3} \\
& +\left(8.471 \times 10^{4}\right) \phi^{2}+\left(-2.854 \times 10^{4}\right) \phi+4.89 \times 10^{3}
\end{aligned}
$$

valid in the range $0.6 \leq \phi \leq 1.6$. The characteristic time of vaporization is of the order of [30]:

$$
t_{r}=\frac{k_{u}}{\rho_{u} S_{L}^{2} C_{T}}
$$


which is the residence time of a coal particle before it enters the reaction zone. It is noted that $S_{L}$ in Eq. (2.41) is actually the laminar propagation velocity of a gaseous flame (without particles) for a given equivalence ratio, and this quantity can be calculated by removing the coal dust particles in Eq. (2.37), i.e. by substituting $C_{T}=C_{p}$ in Eq. (2.38). In this case, to match the calculated laminar flame velocity in the case of no particles, the experimental values of $S_{L}$, given in Table 2.2, are used. The characteristic time of vaporization, $t_{r}$, is used to estimate the total mass of released volatiles per unit volume, $w_{v}=w_{v}^{\prime} t_{r}$. Similar to Ref. [30], for simplicity, this additional fuel is assumed to be methane $\left(\mathrm{CH}_{4}\right)$, which is added to the original gaseous methane/air mixture, thereby promoting the equivalence ratio. The new amount of gaseous fuel per unit volume in the mixture is designated as $m_{\text {fuel }}^{m}=m_{\mathrm{CH}_{4}}^{m}+w_{v}$, where $m_{\mathrm{CH}_{4}}^{m}$ is the original mass of methane per unit volume for a given equivalence ratio, and it can be calculated together with that for air as

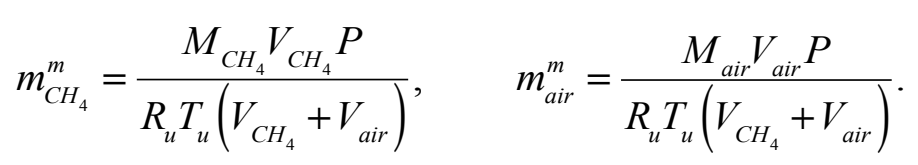

Here $P$ is the atmospheric pressure, $V_{C_{4}}$ and $V_{\text {air }}, M_{C_{4}}$ and $M_{\text {air }}$ are the volumes and molar masses of methane and air, respectively. Accordingly, the modified equivalence ratio can be estimated as:

$$
\phi_{s}=\frac{\left[\left(m_{\text {fuel }}^{m} / M_{C_{4}}\right) /\left(m_{\text {air }}^{m} / M_{\text {air }}\right)\right]_{\text {act }}}{\left[\left(m_{\mathrm{CH}_{4}}^{m} / M_{\mathrm{CH}_{4}}\right) /\left(m_{\text {air }}^{m} / M_{\text {air }}\right)\right]_{s t}} .
$$

With this modified equivalence ratio, a new flame temperature, $T_{f}^{*}$, is estimated by Eq. (2.40). The outcome for a methane/air premixed flame is shown in Fig. 2.11(a), where $T_{f}^{*}$ is presented versus the coal dust concentration $c_{s}$ for various equivalence ratios, $\phi=0.7 ; 0.8 ; 0.9 ; 1$. To match the experimental values of Table 2.1 in the case of no coal-dust particles, Eq. (2.37) is modified as

$$
S_{d, L}=S_{L} \sqrt{\frac{\phi_{s}}{\phi}} \sqrt{\frac{C_{P}}{C_{T}}}\left(\frac{T_{f}}{T_{b}}\right)^{2}\left(\frac{T_{b}-T_{u}}{T_{f}-T_{u}}\right) \sqrt{\exp \left(\frac{E\left(T_{f}-T_{b}\right)}{T_{f} T_{b} R_{u}}\right)}
$$


The corresponding values of $T_{f}^{*}$ are then used to estimate the new laminar flame velocity, $S_{d, L}^{*}$, by substituting $T_{f}^{*}$ instead of $T_{f}$ into Eq. (2.44). The results are shown in Fig. 2.11 for particles of radius $r_{s}=25 \mu \mathrm{m}$. Table 2.4 [67] presents other values used in the present analysis.

It is seen that both $T_{f}^{*}$ and $S_{d, L}^{*}$ grow with the increase in $c_{s}$ and/or $\phi$. It is also noted that while the new flame temperature and laminar flame velocity grow significantly with $c_{s}$ for lean combustion, $\phi=0.7$, these $c_{s}$-dependences weaken with the increase in $\phi$ such that $T_{f}^{*}$ and $S_{d, L}^{*}$ appear almost $c_{s}$-invariant for $\phi=1$. This is due to an effective promotion of the equivalence ratio at $\phi=0.7$ resulting from the increase in the flame temperature $T_{f}^{*}$.

(a)

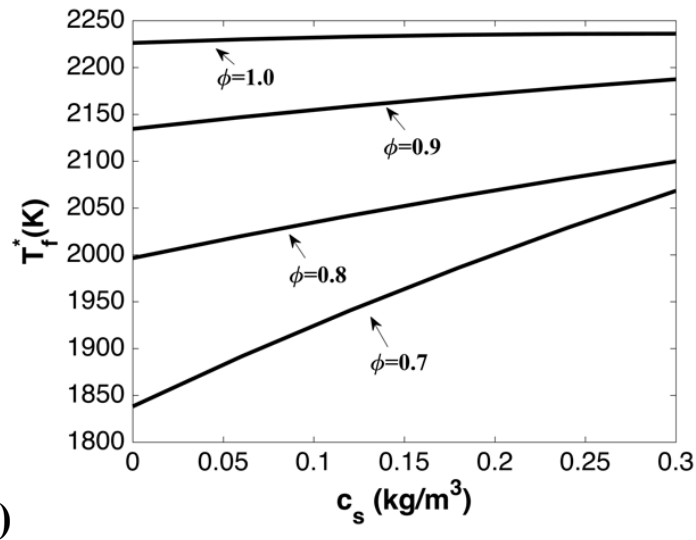

(b)

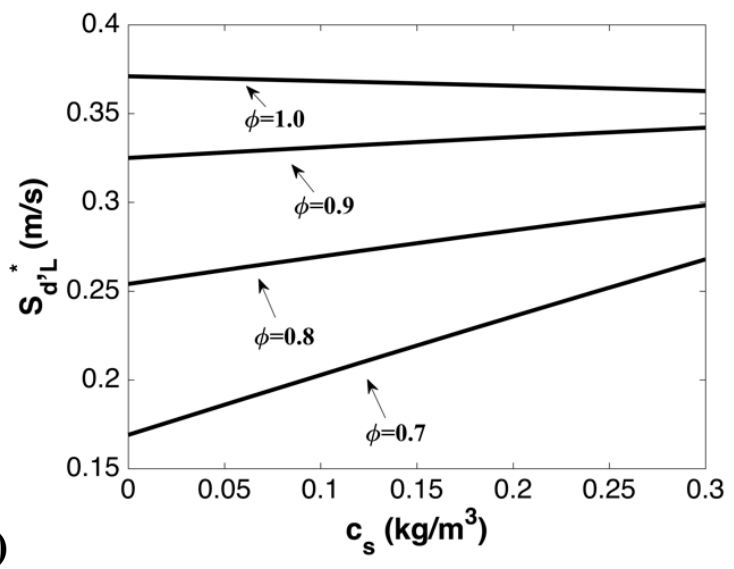

Figure 2.11: The flame temperature $T_{f}^{*}$ (a) and laminar velocity $S_{d, L}^{*}$ (b) modified by the promotion of the equivalence ratio, versus the particle concentration $\mathrm{c}_{\mathrm{s}}$ for the particles of size $r_{s}=25 \mu \mathrm{m}$.

Table 2.4: Some physical parameters used in the study [67].

\begin{tabular}{llll}
\hline$B\left(\mathrm{~s}^{-1}\right)$ & $3.5 \times 10^{6}$ & $\rho_{u}\left(\mathrm{~kg} \mathrm{~m}^{-3}\right)$ & 1.135 \\
$k_{u}\left(\mathrm{~W} \mathrm{~K} \mathrm{~K}^{-1} \mathrm{~m}^{-1}\right)$ & 0.052 & $\rho_{s}\left(\mathrm{~kg} \mathrm{~m}^{-3}\right)$ & 1000 \\
$E_{a}\left(\mathrm{~kJ} \mathrm{~mol}^{-1}\right)$ & 88.8 & $C_{p}\left(\mathrm{~kJ} \mathrm{~kg}^{-1} \mathrm{~K}^{-1}\right)$ & 2.22 \\
$R_{u}\left(\mathrm{~kJ} \mathrm{~mol}^{-1} \mathrm{~K}\right)$ & $8.314 \times 10^{-3}$ & $C_{s}\left(\mathrm{~kJ} \mathrm{~kg}^{-1} \mathrm{~K}^{-1}\right)$ & 1.26 \\
\hline
\end{tabular}


Unlike a combustible (say, coal) particle, an inert (say, sand) particle acts only as a heat sink, because it absorbs heat from the flame and reduces the flame temperature. For lean $(\phi<1)$ or stoichiometric $(\phi=1)$ methane - air combustion, the global chemical reaction reads:

$$
\phi \mathrm{CH}_{4}+2\left(\mathrm{O}_{2}+3.76 \mathrm{~N}_{2}\right) \Rightarrow \phi C \mathrm{O}_{2}+2 \phi \mathrm{H}_{2} \mathrm{O}+7.52 \mathrm{~N}_{2}+4(1-\phi) \mathrm{O}_{2} .
$$

The heat release in the process of burning of $\phi$ moles of methane and 9.52 moles of air equals $\left[\left(T_{b}-T_{u}\right) \sum C_{P} . n_{\text {product }}\right]$. With the assumption that the entire heat released in the reaction is used to raise the temperature of the mixture, the volumetric heat release from methane - air combustion of a given equivalence ratio can be found as:

$$
Q=\left[\left(T_{b}-T_{u}\right) \sum C_{P} \cdot n_{\text {product }}\right] \frac{n_{\text {air }}}{9.52\left(V_{C H_{4}}+V_{\text {air }}\right)},
$$

where $n_{\text {product }}$ is the number of moles of the burning products, which depends on $\phi$. Assuming that a flame with particles releases the same amount of heat while it is also influenced by the temperature rise of particles, a secondly-revised flame temperature, $T_{f}^{* *}$, can be estimated using the energy conservation law:

$$
Q=\left[\left(T_{f}^{* *}-T_{u}\right) \sum C_{P} \cdot n_{\text {product }}\right] \frac{n_{\text {air }}}{9.52\left(V_{C H_{4}}+V_{\text {air }}\right)}+c_{s} C_{s}\left(T_{f}^{* *}+T_{u}\right)+L_{v} .
$$

Rearranging Eq. (2.47), the revised flame temperature is expressed as:

$$
T_{f}^{* *}=\frac{Q-L_{v}}{\frac{n_{\text {air }}}{9.52\left(V_{C H_{4}}+V_{\text {air }}\right)} \sum C_{p} n_{\text {product }}+c_{s} C_{s}}+T_{u},
$$

where $L_{v}$ the heat of gasification per unit volume, which is given by $L_{v}=0.01 w_{v} \Delta h_{C_{4}}[67]$.

Equation (2.48) for the revised flame temperature, which accounts for the heat sink effect, indicates a continous decrease in the flame temperature with the addition of the inert particles. It is subsequently used to estimate the revised laminar flame velocity, $S_{d, L}^{* *}$, by substituting $T_{f}^{* *}$ instead of $T_{f}$ into Eq. (2.44). The results are presented in Fig. 2.12, where the revised flame temperature and laminar flame velocity are shown versus the dust concentration $c_{s}$ for various equivalence ratios, $\phi=0.7 ; 0.8 ; 0.9 ; 1$. It is seen that both $T_{f}^{* *}$ and $S_{d, L}^{* *}$ decrease with $c_{s}$, and 
such a decrease get promoted with $\phi$. At the same time, $T_{f}^{* *}$ and $S_{d, L}^{* *}$ grow with $\phi$. This effect becomes more profound with the increase in $\phi$.

Finally, a combined effect of the increase in the equivalence ratio and that of the heat sink can be accounted by averaging the flame temperature over those associated with both effects separately, $T_{f}^{* * *}=\left(T_{f}^{*}+T_{f}^{* *}\right) / 2$ [67]. It is noted, in this respect, that such a definition of $T_{f}^{* * *}$ is solely based on the work by Xie et al. [67], while an alternative definition would influence the subsequent results quantitatively. In fact, an accurate knowledge of the flame temperature is clearly crucial in predicting the flame velocity accurately, in particular, due to the exponential dependence in Eq. (2.37). Again, the new laminar flame velocity $S_{d, L}^{* * *}$ is calculated by substituting $T_{f}^{* * *}$ instead of $T_{f}$ into Eq. (2.44). Figure 2.13 is a counterpart of Fig. 2.12 for $T_{f}^{* * *}$ and $S_{d, L}^{* * *}$ instead of $T_{f}^{* *}$ and $S_{d, L}^{* *}$, respectively. Similar to Fig. 2.12, the increase in $\phi$ promotes both $T_{f}^{* * *}$ and $S_{d, L}^{* * *}$

(a)

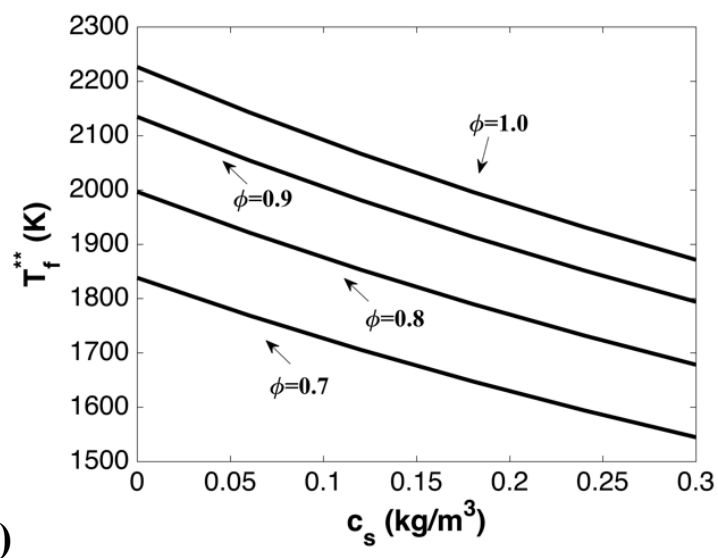

(b)

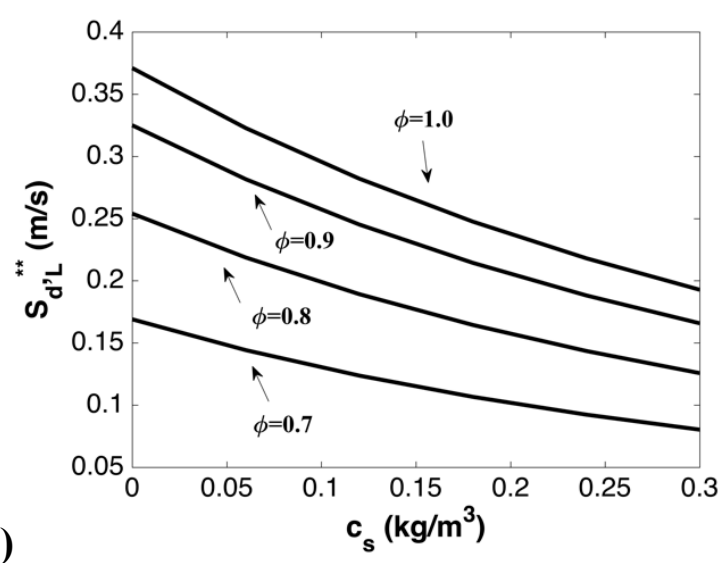

Figure 2.12: The flame temperature $T_{f}^{* *}(\mathrm{a})$ and laminar velocity $S_{d, L}^{* *}$ (b), modified by the heat sink effect, versus the particle concentration $\mathrm{c}_{\mathrm{s}}$ for the particles of size $r_{s}=25 \mu \mathrm{m}$.

However, the dependence on the dust concentration changes: namely, while the $c_{s}$-dependences of $T_{f}^{* * *}$ and $S_{d, L}^{* * *}$ resemble that of $T_{f}^{* *}$ and $S_{d, L}^{* *}$ for stoichiometric combustion, Fig. 2.12, these near-liner dependences weaken with the decrease in $\phi$ such that the effects of the promoted equivalence ratio and heat sink are practically balanced for $\phi=0.7$, making thereby $T_{f}^{* * *}$ and 
$S_{d, L}^{* * *}$ almost $c_{s}$-invariant. This result is opposite to that observed in Fig. 2.11 for $T_{f}^{*}$ and $S_{d, L}^{*}$. Besides, in the case of no particles $\left(c_{s}=0\right)$, all our results for the flame velocity reproduce the experimental data of Table 2.2 .

(a)

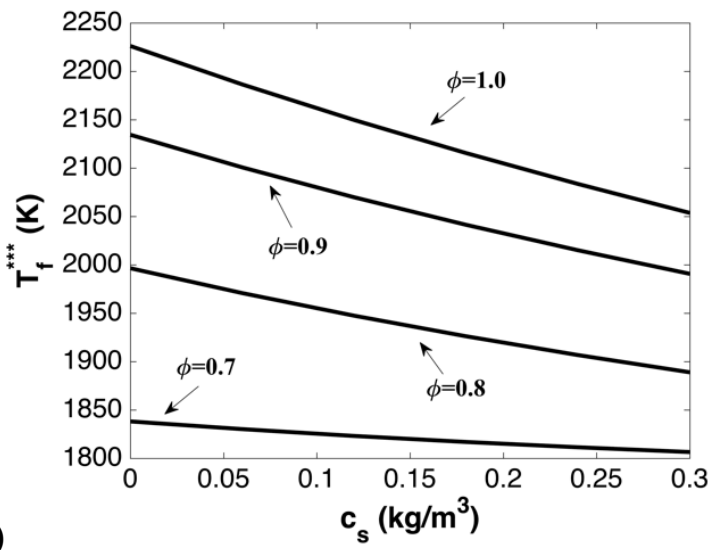

(b)

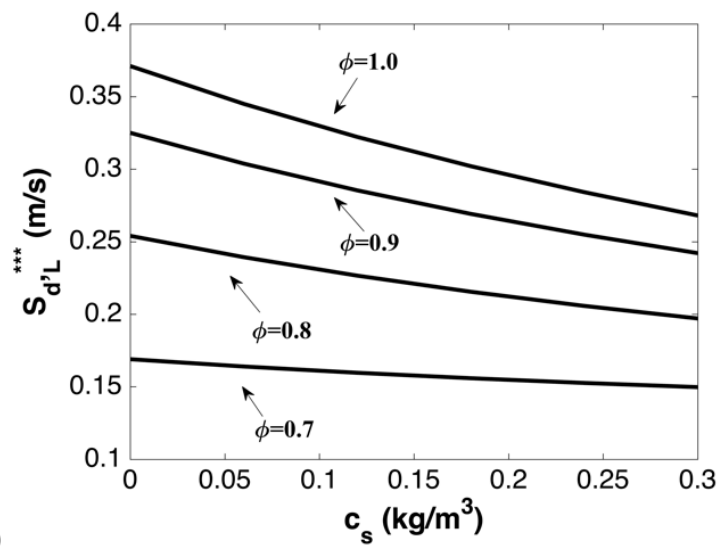

Figure 2.13: The flame temperature $T_{f}^{* * *}$ (a) and laminar velocity $S_{d, L}^{* * *}$ (b), modified to the combined (combustible + inert) effect, versus the particle concentration $\mathrm{c}_{\mathrm{s}}$ for the particles of size $r_{s}=25 \mu \mathrm{m}$.

The effect of a particle radius on the laminar flame velocity in the case of both combustible and combined (combustible + inert) particles is shown in Fig. 2.14, for the fixed equivalence ratio $\phi=0.7$ and various particle radii $r_{s}=10 ; 25 ; 75 \mu \mathrm{m}$, consistent with the realistic coal particle sizes, in both cases. In the case of combustible particles, the laminar flame velocity attains its highest value for $r_{s}=10 \mu \mathrm{m}$. This can be devoted to the effect of faster pyrolysis in small coal particles, which causes an effective increase in an equivalence ratio; see Eq. (2.43). However, the growth of $S_{d, L}^{*}$ moderates around $\mathrm{c}_{\mathrm{s}}=180 \mathrm{~g} / \mathrm{m}^{3}$ and becomes almost constant close to a value of $\mathrm{c}_{\mathrm{s}}=250 \mathrm{~g} / \mathrm{m}^{3}$. This decreasing trend in the slope of $S_{d, L}^{*}$ is mainly caused by an effective increase in the equivalence ratio from $\phi=0.7$ at $c_{s}=0$ till $\phi=1.05$ at $c_{s}=300 \mathrm{~g} / \mathrm{m}^{3}$. Such a strong increase in $\phi$ shows a decreasing slope in the laminar flame velocity when it approaches the stoichiometric value. This observation can also be justified by remembering how the adiabatic flame temperature changes with the equivalence ratio. On the other hand, small particles, $r_{s}=10 \mu \mathrm{m}$, promote the laminar flame velocity $S_{d, L}^{* * *}$ as long as the concentration does not exceed $c_{s}=180 \mathrm{~g} / \mathrm{m}^{3}$, i.e. as long as the heat sink effect plays a minor role. However, with the increase 
in the particle concentration, the heat sink effect becomes dominant and causes a decreasing trend in $S_{d, L}^{* * *}$. Besides, relatively larger particles, with $r_{s}=25 \mu \mathrm{m}$ and $r_{s}=75 \mu \mathrm{m}$, monotonically reduce the flame velocity with the increase in the particle concentration.
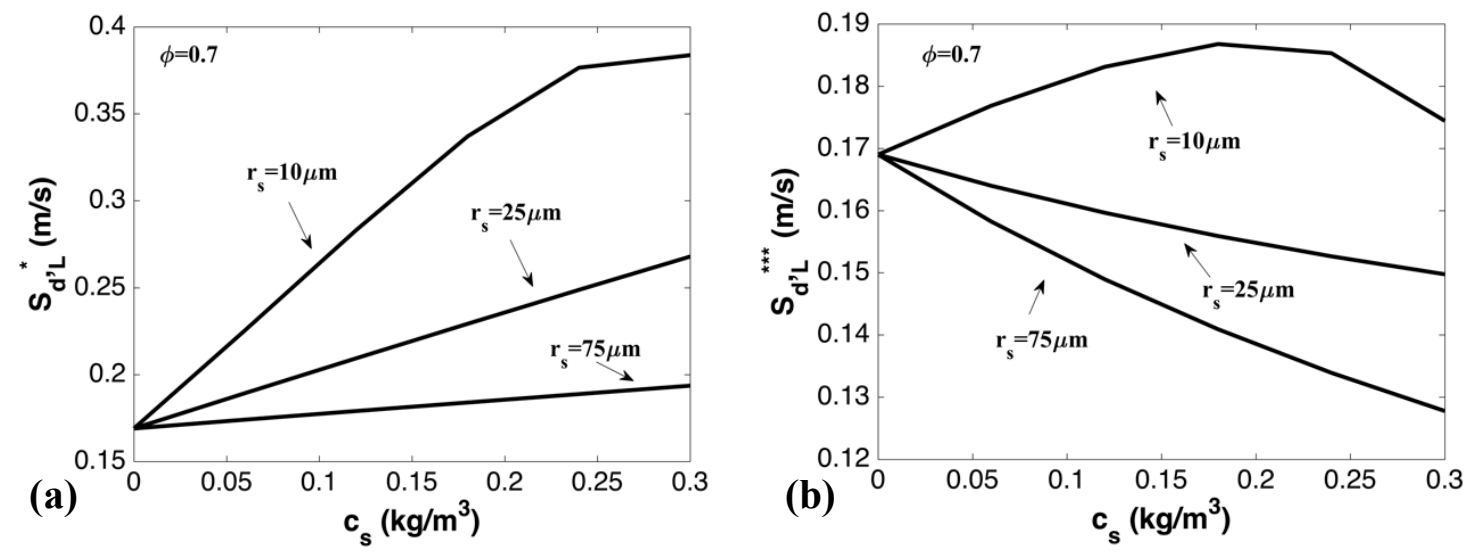

Figure 2.14: The modified flame velocity versus the particle concentration for the fixed equivalence ratio $(\phi=0.7)$ and various particle radii $\left(r_{s}=10 ; 25\right.$ and $\left.75 \mu \mathrm{m}\right)$ for combustible (a) and combined (combustible + inert) (b) particles.

Next, these updates on the laminar flame velocity are incorporated into the formulation of Sec. 2.1 on gaseous combustion, thereby modifying the results of Sec. 2.2. Specifically, the scenarios of globally-spherical flame expansion and that of finger flame acceleration accounting for the presence of combustible and/or inert particles are updated both for planar and cylindrical geometries. As the modified equivalence ratios and the modified laminar flame velocities are incorporated into the formulations of Sec. 2.1, all the functions of the equivalence ratio and laminar flame velocity will change accordingly, and they also modify the timing and key characteristics of the flame dynamics such as the evolution of the flame tip position and velocity.

Figures 2.15 and 2.16 compare the situations of combustible, inert and both types of particles as well as that with no particles. Specifically, Fig. 2.15(a) presents the evolution of the flame tip for methane/air/dust burning of equivalence ratio $\phi=0.7$ and particle concentration $\mathrm{c}_{\mathrm{s}}=120 \mathrm{~g} / \mathrm{m}^{3}$ for the 2D planar and cylindrical axisymmetric geometries. The particles of two different sizes, $r_{s}=75 \mu \mathrm{m}$ and $r_{s}=10 \mu \mathrm{m}$, are employed in Figs. 2.15(a) and 2.15(b), respectively. Figures 2.16(a) and 2.16(b) are respectively the counterparts of Figs. 2.15(a) and 2.15(b) for the flame tip velocity. Figures 2.15 and 2.16 justify the expectation that flame propagation is facilitated by 
combustible dust but it is mitigated by inert dust. It is also noted that small particles, $r_{s}=10 \mu \mathrm{m}$, Figs. 2.15(b) and 2.16(b), both combustible and combustible + inert, impact flame propagation noticeably stronger than that of $r_{s}=75 \mu \mathrm{m}$, Figs. 2.15(a) and 2.16(a). In particular, while the difference between the cases of no particles and combustible particles is hardly seen for $r_{s}=75 \mu \mathrm{m}$ in the cylindrical geometry, such a difference is substantial for $r_{s}=10 \mu \mathrm{m}$, both in the 2D-planar and cylindrical-axisymmetric configurations. The difference between the cases of no particles and that of combined particles becomes relatively small in the cylindrical geometry. Additionally, in accordance with Sec. 2.2, the flame tip velocity attains higher values for the all (combustible, inert, combined combustible + inert, and no particles) cases in the cylindrical geometry as compared to the $2 \mathrm{D}$ one, hence yielding faster flame propagation. Such a qualitative and quantitative difference is demonstrated in Figs. 2.15 and 2.16.
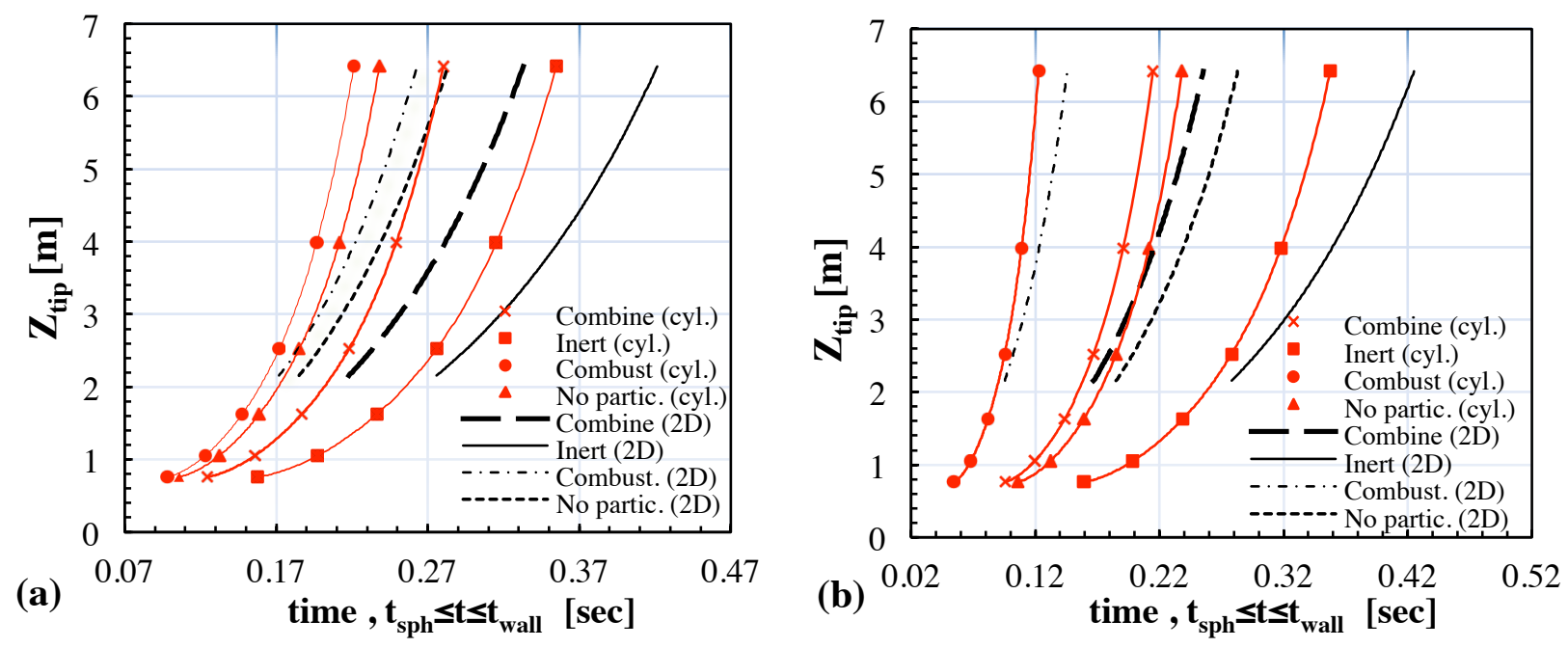

Figure 2.15: Evolution of flame tip position $Z_{\text {tip }}$ in a homogeneous gaseous environment and in the presence of combustible, inert, and combined (combustible + inert) particles in both 2Dplanar and cylindrical geometries for $\phi=0.7, \mathrm{c}_{\mathrm{s}}=120 \mathrm{~g} / \mathrm{m}^{3}$ and $r_{s}=75 \mu \mathrm{m}$ (a), $r_{s}=10 \mu \mathrm{m}$ (b). 

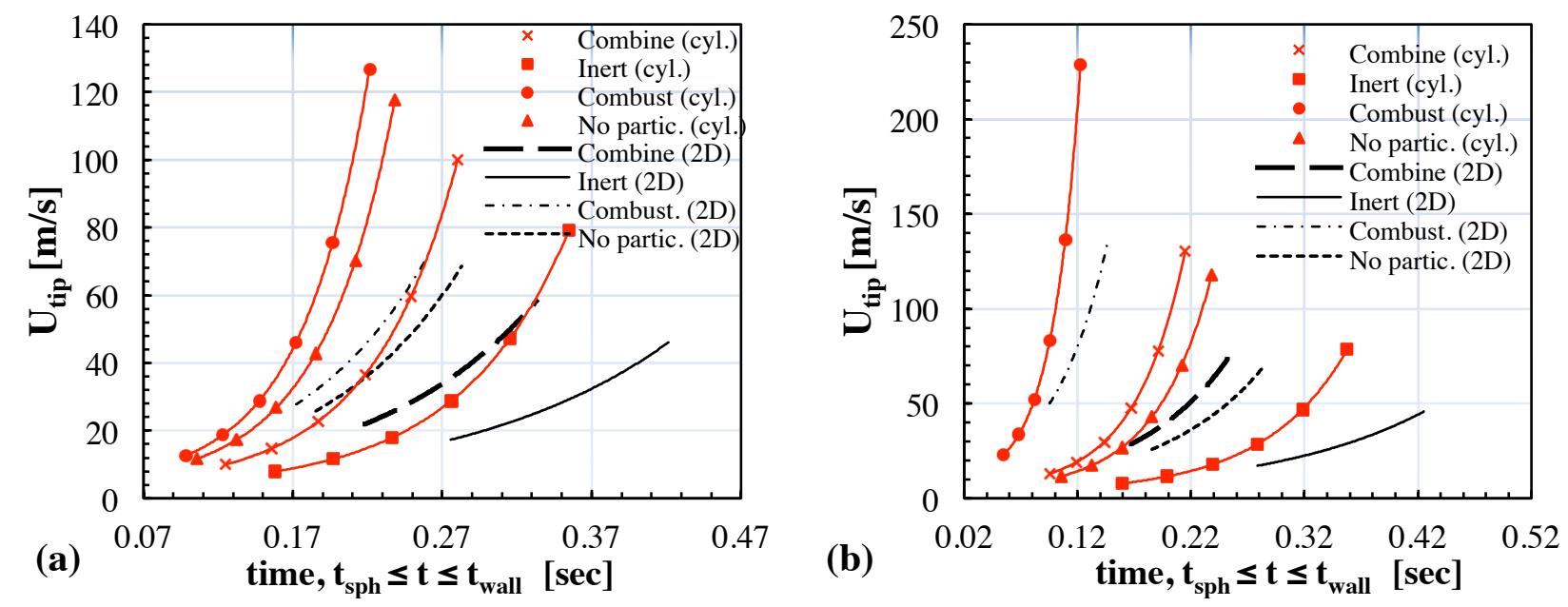

Figure 2.16: Evolution of flame tip position $U_{t i p}$ in a homogeneous gaseous environment and in the presence of combustible, inert, and combined (combustible + inert) particles in both 2Dplanar and cylindrical geometries for $\phi=0.7, \mathrm{c}_{\mathrm{s}}=120 \mathrm{~g} / \mathrm{m}^{3}$ and $r_{s}=75 \mu \mathrm{m}$ (a), $r_{s}=10 \mu \mathrm{m}$ (b).
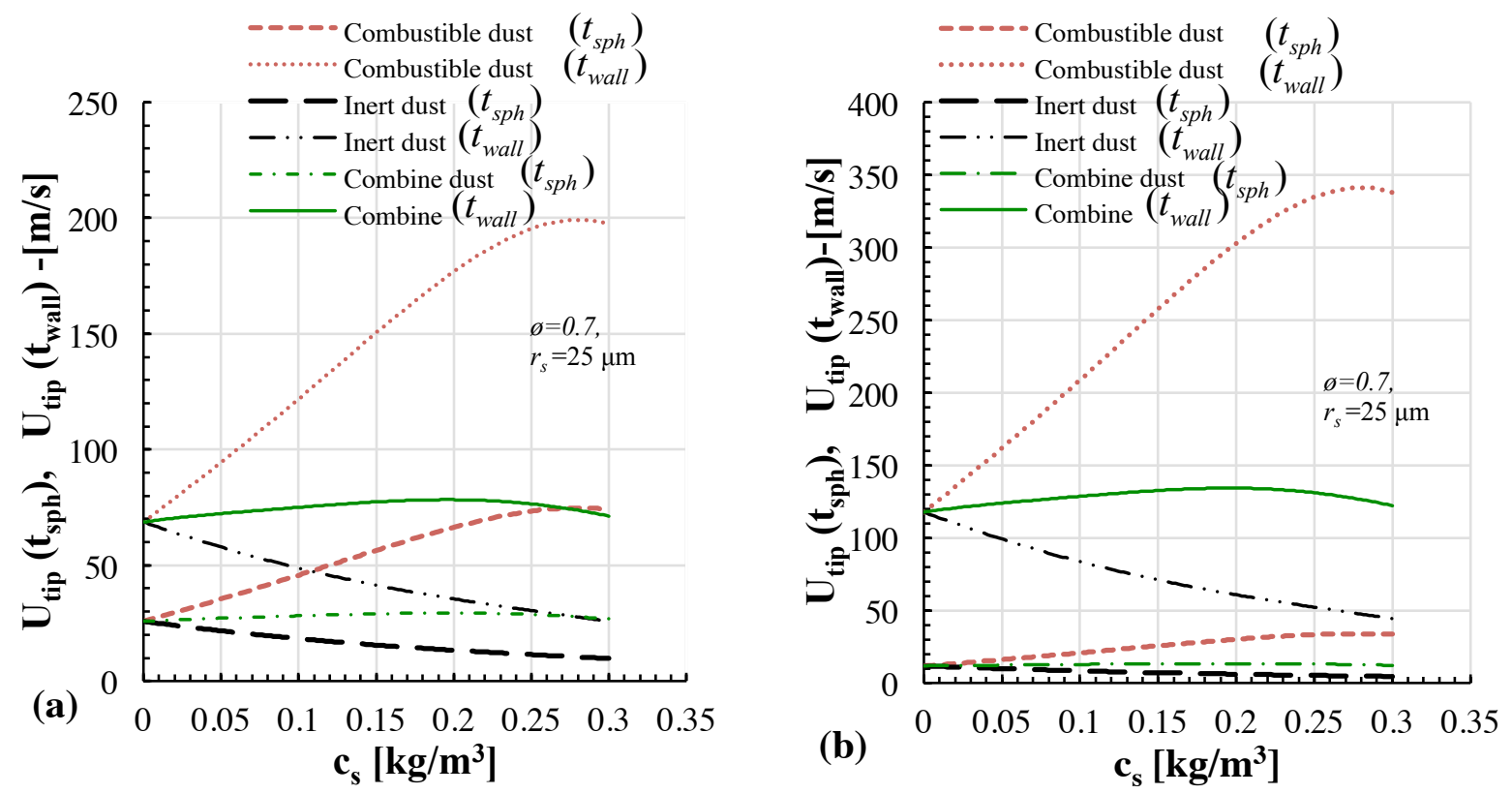

Figure 2.17: Instantaneous flame tip velocities $U_{\text {tip }}\left(t_{\text {sph }}\right)$ and $U_{\text {tip }}\left(t_{\text {wall }}\right)$ versus the particle concentration $\mathrm{c}_{\mathrm{s}}$ for combustible, inert and combined particles for $\phi=0.7$ and $r_{s}=25 \mu \mathrm{m}$ in the 2D-planar (a) and cylindrical-axisymmetric (b) geometries.

The effect of particles concentration on the instantaneous flame tip velocity $U_{\text {tip }}\left(t_{\text {sph }}, t_{\text {wall }}\right)$ is investigated for the combustible, inert and combined combustible + inert dust for the equivalence 
ratio $\phi=0.7$ and the particle radius $r_{s}=25 \mu \mathrm{m}$. The result is shown in Fig. 2.17, for the 2D (a) and cylindrical (b) geometries. Specifically, the flame tip velocities attained at the time instants $t_{s p h}$ and $t_{\text {wall }}$ increase with the concentration of the combustible dust as long as the $\mathrm{c}_{\mathrm{s}}<250 \mathrm{~g} / \mathrm{m}^{3}$. In contrast, the flame tip velocity diminishes with the concentration of the inert dust. For a given particle size and equivalence ratio, the increase or decrease in the combined particles concentration does not influence $U_{t i p}\left(t_{s p h}\right)$. For the same particle type, $U_{\text {tip }}\left(t_{\text {wall }}\right)$ slightly increases with the concentration while $c_{\mathrm{s}}<200 \mathrm{~g} / \mathrm{m}^{3}$ but then decreases when $\mathrm{c}_{\mathrm{s}}>200 \mathrm{~g} / \mathrm{m}^{3}$.

\subsection{Effect of Gas Compressibility}

It is noted that the pilot theory presented through Secs. 2.1-2.3 did not account for certain factors occurring in a real coal mine such as turbulence, shock waves and spatial/temporal variations of the combustible premixture. Most importantly, the formulation presented in Sec. 2.1 employed the approach of an incompressible flow, and it was shown that in a 2D geometry, no detonation initiation was predicted for methane combustion. However, in the case of propane, the detonation occurs for fuel-rich burning, with the equivalence ratios $\phi$ in the range $1 \leq \phi \leq 1.2$. For the cylindrical geometry, this range varied as $0.9 \leq \phi \leq 1.2$ and $0.8 \leq \phi \leq 1.3$ for methane and propane, respectively. Although propane/air combustion is not the case in coal mines, it was included into the previous and present analyses for comparison purposes. A threshold condition for DDT is that the maximal attainable burning velocity should be high enough to generate a sufficiently strong shock wave ahead of the flame front to cause an auto-ignition of the compressed reactants between the shock and the flame [59]. Experimental studies [68, 69] and numerous computational simulations of DDT [21, 70] have shown that even for highly-explosive mixtures, such as hydrogen/oxygen or ethylene/oxygen, an exponential increase of the flame tip velocity stopped much earlier than the sound barrier.

However, while the incompressible scenario is acceptable to describe the initial stages of burning, gas compression certainly becomes substantial by the end of the "finger" stage, when the velocity of the flame front may start approaching a near-sonic value. It is recalled, in this respect, that gas compression moderates flame acceleration in various configurations [19, 57, 
71], hence, the same effect is anticipated in the coalmining geometry, and verifying this statement constitutes the focus of this section. Specifically, the role of gas compressibility in the event of finger flame acceleration in a coalmining passage is quantified, thereby validating the incompressible formulations and identifying its limitations. Similar to Sec. 2.1, here the 2Dplanar and cylindrical-axisymmetric configurations are considered. Accordingly, starting with gaseous combustion, the analysis is then extended to the gaseous-dusty flows. The impacts of the equivalence ratio as well as that of the size and concentration of the coal dust particles on the flame evolution are systematically investigated.

\subsubsection{D Planar Geometry}

Next the analytical formulation of Sec. 2.1.1 is extended to account for small but finite Mach numbers based on the methodology of Ref. [57]. However, while a Mach number associated with flame propagation, $M a=S_{L} / c_{0}$, with $c_{0}$ being the initial sound speed in the fresh mixture, was constant in Ref. [57], here $M a$ depends on time as

$$
M a(t)=U_{D L}(t) / c_{0}=(n C / \Theta) t^{n-1} / c_{0},
$$

where $U_{D L}(t)$ is given by Eq. (2.5). The effect of compressibility becomes significant when $M a \geq 0.3$. As soon as the combustion process is substantially subsonic, say, $M a \leq 0.3$, the flow in the unburnt gas can be treated as isentropic, with the instantaneous density, pressure and temperature given by $[57,72]$

$$
\begin{aligned}
& \frac{\rho_{u}(t)}{\rho_{0}}=\left(1+\frac{\gamma-1}{2} \frac{u_{z, 1}(t)}{c_{0}}\right)^{2 /(\gamma-1)} \approx 1+M a(t)(\Theta-1)\left(1+\frac{Z_{t i p}(t)}{H}\right), \\
& \frac{P_{u}(t)}{P_{0}}=\left(1+\frac{\gamma-1}{2} \frac{u_{z, 1}(t)}{c_{0}}\right)^{2 \gamma /(\gamma-1)} \approx 1+\gamma M a(t)(\Theta-1)\left(1+\frac{Z_{t i p}(t)}{H}\right), \\
& \frac{T_{u}(t)}{T_{0}}=\left(1+\frac{\gamma-1}{2} \frac{u_{z, 1}(t)}{c_{0}}\right)^{2} \approx 1+(\gamma-1) M a(t)(\Theta-1)\left(1+\frac{Z_{t i p}(t)}{H}\right),
\end{aligned}
$$

where $\gamma=C_{p} / C_{v} \approx 1.4$ is the adiabatic index (or heat capacity ratio) and $\rho_{0}, P_{0}, T_{0}$ the initial values in the unburnt gas. Instead of the initial thermal expansion $\Theta$, one now deals with an instantaneous (reduced) thermal expansion factor in the form: 


$$
\vartheta(t)=\Theta-(\gamma-1) M a(t)(\Theta-1)^{2}\left\{1+Z_{t i p}(t) / H\right\} .
$$

The $2 \mathrm{D}$ continuity equation for small but finite compressibility, $\nabla \mathbf{u}=-\left(\partial P_{u} / \partial t\right) / \gamma P_{u}$, has the solution in the burnt gas

$$
u_{x}=-(\vartheta-1) \frac{U_{D L}(t)}{H} x, \quad u_{z}=\left(-\frac{1}{\gamma P_{u}} \frac{\partial P_{u}}{\partial t}+(\vartheta-1) \frac{U_{D L}(t)}{H}\right) z .
$$

Substituting Eqs. (2.49) - (2.54) into a modified Eq. (2.14), $\dot{Z}_{t i p}-u_{z, 2}(\vartheta(t))=\vartheta(t) U_{D L}(t)$, and further neglecting the $2^{\text {nd }}$-and higher order terms in $M a$, eventually the evolution equation can be found in the form:

$$
\begin{gathered}
\frac{d Z_{t i p}}{d t}=\left[-\frac{n(n-1) C t^{n-2}}{\Theta c_{0}}(\Theta-1)\left(1+\frac{Z_{t i p}}{h}\right)+\frac{n C t^{n-1}}{\Theta c_{0}}\left[-\left(\frac{\Theta-1}{H}\right) \frac{n C t^{n-1}}{\Theta}\left((\Theta-1)\left(\frac{Z_{t i p}}{H}\right)+\Theta\right)\right]\right. \\
\left.+\left((\Theta-1)-\frac{n C t^{n-1}}{\Theta c_{0}}(\gamma-1)(\Theta-1)^{2}\left(1+\frac{Z_{t i p}}{H}\right)\right) \frac{n C t^{n-1}}{\Theta H}\right] Z_{t i p}+ \\
\left(\Theta-\frac{n C t^{n-1}}{\Theta c_{0}}(\gamma-1)(\Theta-1)^{2}\left(1+\frac{Z_{t i p}}{H}\right)\right) \frac{n C t^{n-1}}{\Theta} .
\end{gathered}
$$

\subsubsection{Cylindrical Axisymmetric Geometry}

Similarly, the cylindrical-axisymmetric formulation of Sec. 2.1.2 can be extended to include compressibility in the same manner as in a 2D-planar geometry. Namely, the flow in the unburnt gas can be treated as isentropic, and the instantaneous density, pressure and temperature read:

$$
\begin{aligned}
& \frac{\rho_{u}(t)}{\rho_{0}}=\left(1+\frac{\gamma-1}{2} \frac{u_{z, 1}(t)}{c_{0}}\right)^{2 /(\gamma-1)} \approx 1+\operatorname{Ma}(t)\left(2 \alpha \frac{Z_{t i p}(t)}{H}+\Theta-1\right), \\
& \frac{P_{u}(t)}{P_{0}}=\left(1+\frac{\gamma-1}{2} \frac{u_{z, 1}(t)}{c_{0}}\right)^{2 \gamma /(\gamma-1)} \approx 1+\operatorname{Ma}(t) \gamma\left(2 \alpha \frac{Z_{t i p}(t)}{H}+\Theta-1\right), \\
& \frac{T_{u}(t)}{T_{0}}=\left(1+\frac{\gamma-1}{2} \frac{u_{z, 1}(t)}{c_{0}}\right)^{2} \approx 1+\operatorname{Ma}(t)(\gamma-1)\left(2 \alpha \frac{Z_{t i p}(t)}{H}+\Theta-1\right) .
\end{aligned}
$$

with the instantaneous (reduced) thermal expansion in the form

$$
\vartheta(t)=\Theta-(\gamma-1) M a(t)(\Theta-1)^{2}\left\{1+2 \sqrt{\Theta /(\Theta+1)} Z_{t i p}(t) / H\right\} .
$$


The axisymmetric continuity equation for small but finite compressibility, $\nabla \mathbf{u}=-\left(\partial P_{u} / \partial t\right) / \gamma P_{u}$, has the following solution in the burnt gas:

$$
u_{r}=-(\vartheta-1) \frac{U_{D L}(t) R_{f}}{H^{2}} r, \quad u_{z}=\left(-\frac{1}{\gamma P_{u}} \frac{\partial P_{u}}{\partial t}+2(\vartheta-1) \frac{U_{D L}(t) R_{f}}{H^{2}}\right) z .
$$

Substituting Eqs (2.49), (2.56) - (2.60) into a modified Eq. (2.22), $\dot{Z}_{t i p}-u_{z, 2}(\vartheta(t))=\vartheta(t) U_{D L}(t)$, and further neglecting the $2^{\text {nd }}$ - and higher order terms in $M a$, the evolution equation is found as

$$
\begin{aligned}
\frac{d Z_{t i p}}{d t}=\left[-\frac{n(n-1) C t^{n-2}}{\Theta c_{0}}\left(2 \alpha \frac{Z_{t i p}}{H}+\Theta-1\right)+\frac{n C t^{n-1}}{\Theta c_{0}}\left[-\left(\frac{n C t^{n-1} 2 \alpha}{\Theta H}\right)\left(2 \alpha \frac{Z_{t i p}}{H} \tanh \left(\frac{\alpha C t^{n}}{\Theta H}\right)+\Theta\right)\right]\right. \\
+\frac{n C t^{n-1}}{\Theta H}\left[2 \alpha \tanh \left(\frac{\alpha C t^{n}}{\Theta H}\right)-\frac{n C t^{n-1}}{\Theta H}(2 \Theta-1)(\gamma-1)(\Theta-1)^{2}\left(1+\frac{2 \Theta}{\alpha} \frac{Z_{t i p}}{H}\right) \times\right. \\
{\left.\left.\left[\frac{C t^{n}}{\Theta H} \operatorname{sech}\left(\frac{\alpha C t^{n}}{\Theta H}\right)+\frac{1}{2 \alpha} \tanh \left(\frac{\alpha C t^{n}}{\Theta H}\right)\right]\right]\right] Z_{t i p}+} \\
\left(\Theta-\frac{n C t^{n-1}}{\Theta c_{0}}(\gamma-1)(\Theta-1)^{2}\left(1+\frac{2 \Theta}{\alpha} \frac{Z_{t i p}}{H}\right)\right) \frac{n C t^{n-1}}{\Theta} .
\end{aligned}
$$

Moreover, Eqs. (2.12) and (2.25) for $t_{s p h}$ and Eqs. (2.16) and (2.29) for $t_{\text {wall }}$ also can be extended to account for gas compression by replacing $\Theta$ with $\vartheta$ such that a flame front contacts the passage wall slightly later. However, such an effect was shown to be minor [57]; hence, it is neglected here.

\subsection{Results and Discussion (Compressible Flow)}

Equations (2.55) and (2.61) describe the effect of gas compression on finger-flame acceleration in a mining passage for the 2D-planar and cylindrical-axisymmetric geometries, respectively. These equations have been solved numerically by using a $4^{\text {th }}$-order Runge-Kutta iterative method and compared to the incompressible 2D-planar, Eqs. (2.15) - (2.18) and cylindrical-axisymmetric, Eqs. (2.28) - (2.31), formulations, in Figs. 2.18, 2.19, 2.20 and 2.21, with $n=1.4, R=H=1.05 \mathrm{~m}$, and various methane- and propane/air equivalence ratios, $0.63 \leq \phi$ $\leq 1.4$, in all figures. Specifically, Figures 2.18(a), 2.19(a), 2.20(a) and 2.21(a) present the flame 
tip evolution $Z_{t i p}(t)$, while Figs. 2.18(b), 2.19(b), 2.20(b) and 2.21(b) show $U_{t i p}(t)$ in the interval $t_{s p h} \leq t \leq t_{w a l l}$, i.e. during the entire finger flame scenario for methane- and propane/air burning.

As anticipated, gas compression moderates the acceleration process in all cases. Still, the effect is relatively minor for lean and rich mixtures, but it is substantial for near-stoichiometric combustion associated with the strongest acceleration and highest flame speeds. Specifically, in a 2D-planar geometry, Figs. 2.18(a) and 2.19(a), for $\phi=0.63, \phi=1$ and $\phi=1.4$, the compressibility moderates the maximal flame tip position by $5 \%, 43 \%$ and $15 \%$, respectively, for methane/air flames; and by $12 \%, 53 \%$ and $31 \%$, respectively, for propane/air combustion. On the other hand, for the cylindrical geometry, Figs. 2.20(a) and 2.21(a), the relative reduction of the maximal $Z_{\text {tip }}$ due to gas compression is stronger, being $11 \%, 61 \%$ and $27 \%$, for methane, and $22 \%, 69 \%$ and $47 \%$, for propane, for $\phi=0.63, \phi=1$ and $\phi=1.4$, respectively, in both media.

This effect is even more notable in Figures 2.18(b) and 2.19(b), for the flame tip velocity, $U_{t i p}$, which decreases in a 2D-planar geometry from $288 \mathrm{~m} / \mathrm{s}$ till $69 \mathrm{~m} / \mathrm{s}$ for the stoichiometric methane/air flames, and from $388 \mathrm{~m} / \mathrm{s}$ till $69 \mathrm{~m} / \mathrm{s}$ for stoichiometric propane/air combustion. In the cylindrical case, Figs. 2.20(b) and 2.21(b), the effect is even stronger: the maximal $U_{\text {tip }}$ decreases from $498 \mathrm{~m} / \mathrm{s}$ till $70 \mathrm{~m} / \mathrm{s}$ for stoichiometric methane/air burning, and from $674 \mathrm{~m} / \mathrm{s}$ till $66 \mathrm{~m} / \mathrm{s}$ for the stoichiometric propane/air flames. Among other important consequences of the developed compressible analysis, it is noted that the stoichiometric methane- or propane/air mixtures show a deceleration trend in Figs. 2.18(b), 2.19(b), 2.20(c) and 2.21(b) for $U_{\text {tip }}$ before the flame skirt contacting the passage sidewalls. In fact, this deceleration (shown by the dotted lines) is beyond the limitations of the present theory (assuming a small but finite $M a$ ) and thereby it is associated with a numerical artifact. 

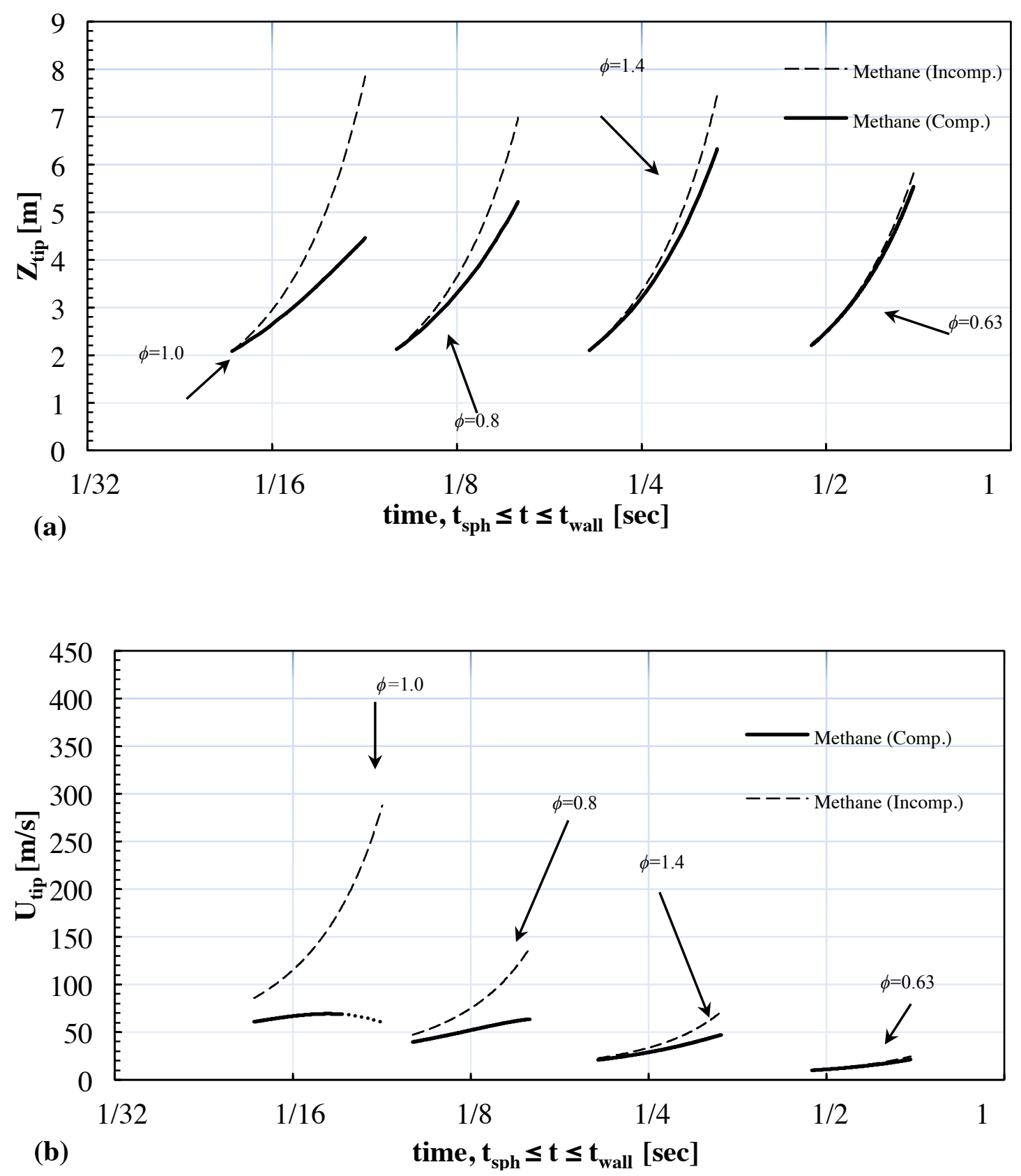

Figure 2.18: 2D-planar geometry: Comparison of the incompressible and compressible formulations: Evolution of the flame tip position $Z_{\text {tip }}$ (a) and its velocity $U_{t i p}$ (b) for the methane/air flames of various equivalence ratios: $\phi=0.63 ; 0.8 ; 1 ; 1.4$. 

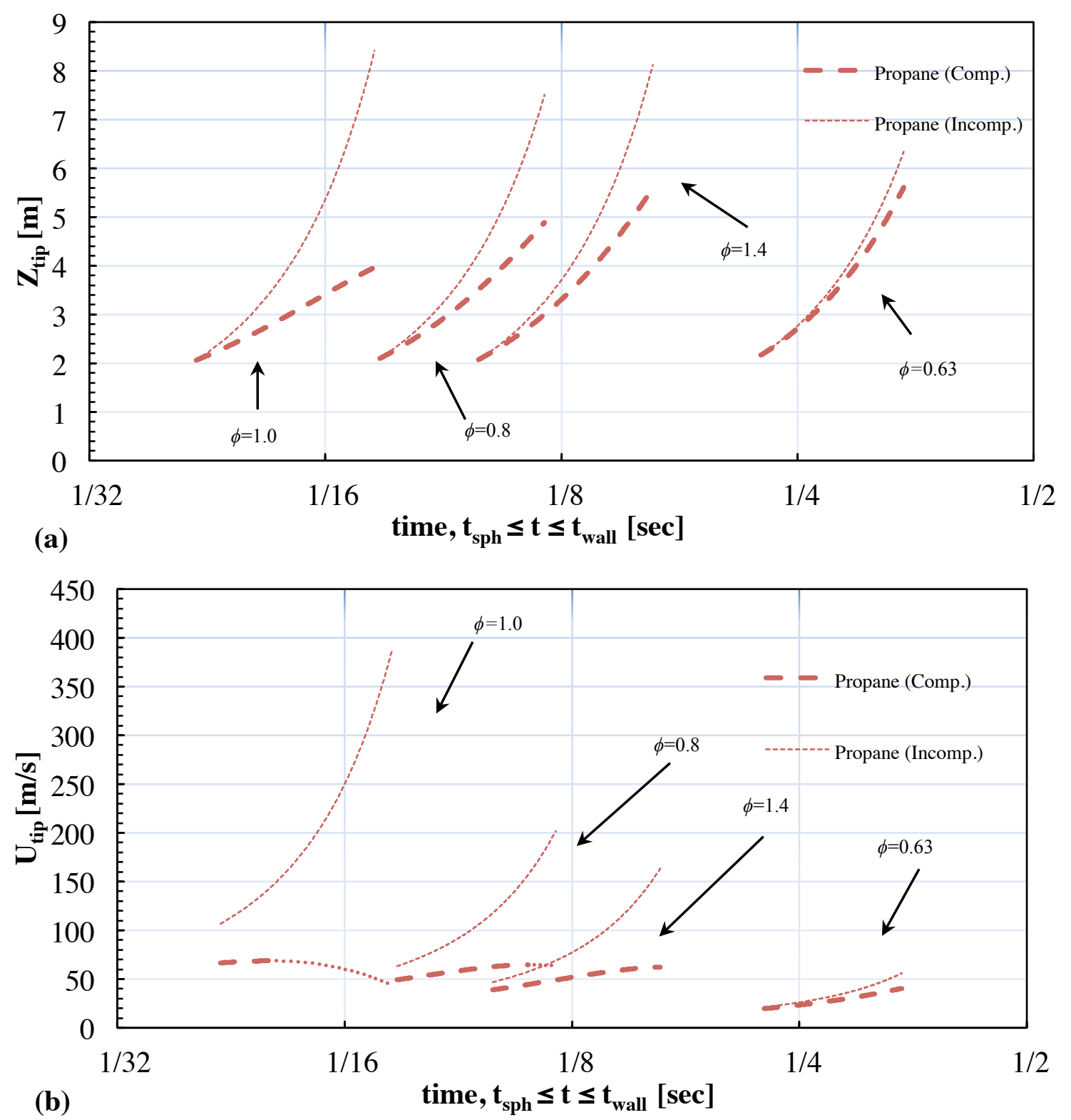

Figure 2.19: 2D-planar geometry: Comparison of the incompressible and compressible formulations: Evolution of the flame tip position $Z_{t i p}$ (a) and its velocity $U_{t i p}$ (b) for the propane/air flames of various equivalence ratios: $\phi=0.63 ; 0.8 ; 1 ; 1.4$. 

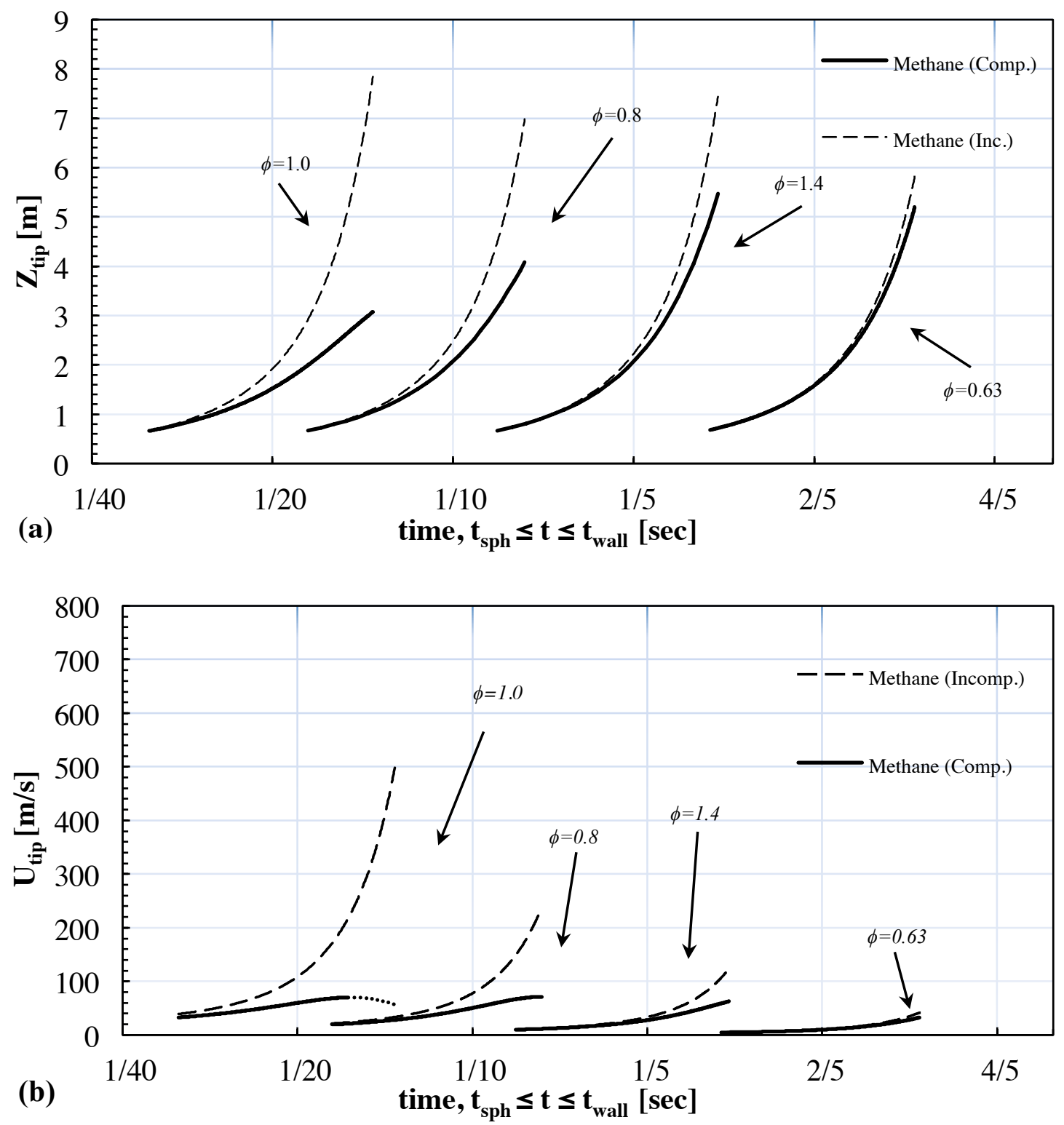

Figure 2.20: Cylindrical-axisymmetric geometry: Comparison of the incompressible and compressible formulations: Evolution of the flame tip position $Z_{\text {tip }}$ (a) and its velocity $U_{\text {tip }}$ (b) for methane/air flames of various equivalence ratios: $\phi=0.63 ; 0.8 ; 1 ; 1.4$. 

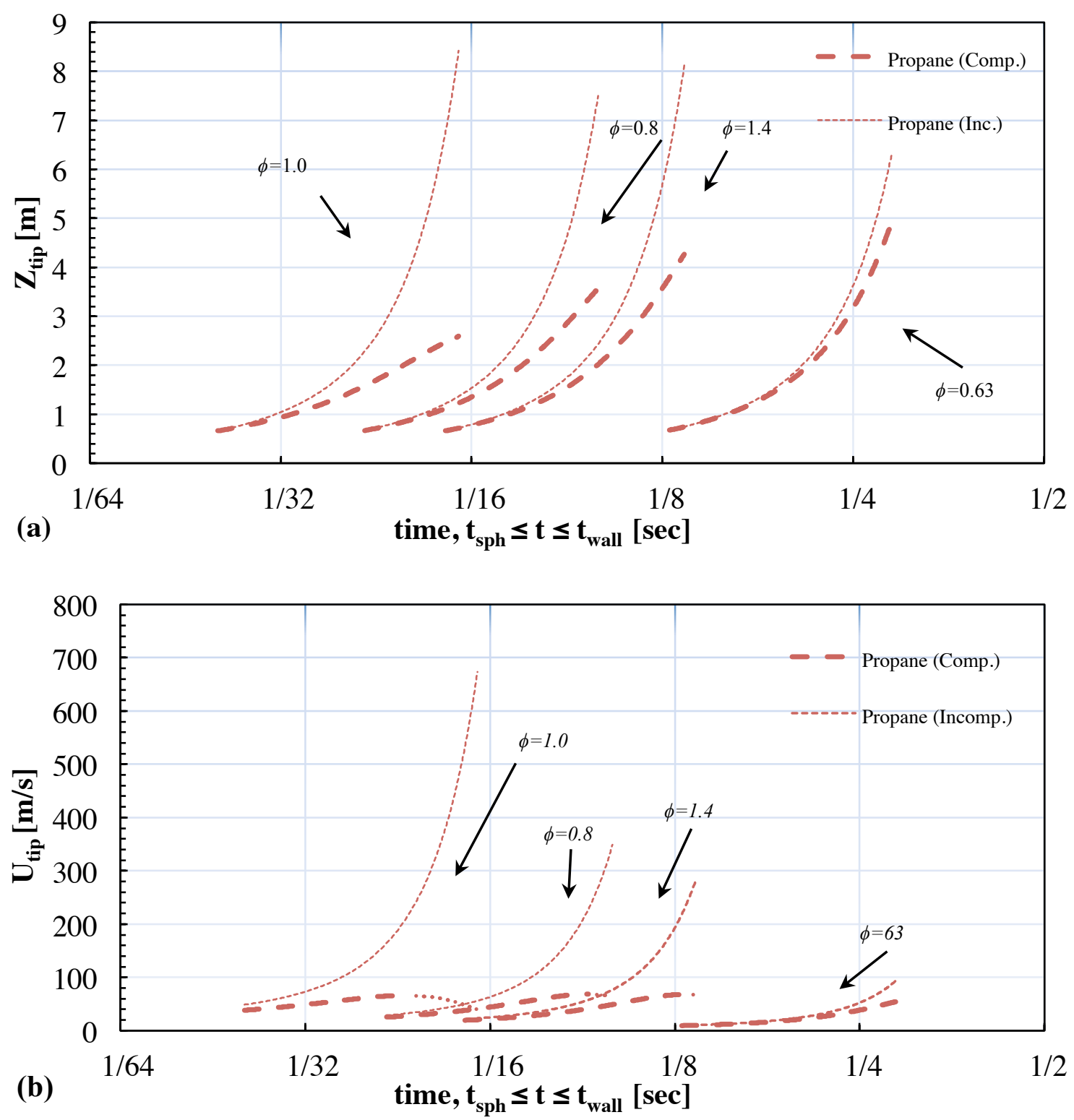

Figure 2.21: Cylindrical-axisymmetric geometry: Comparison of the incompressible and compressible formulations: Evolution of the flame tip position $Z_{t i p}$ (a) and its velocity $U_{\text {tip }}$ (b) for the propane/air flames of various equivalence ratios: $\phi=0.63 ; 0.8 ; 1 ; 1.4$.

Figure 2.22 compares the 2D-planar and cylindrical-axisymmetric geometries for the same thermal-chemical methane/air parameters. Specifically, the flame tip position, $Z_{t i p}$, Fig. 2.22(a), and velocity, $U_{t i p}$, Fig. 2.22(b), are compared in the cases of lean $(\phi=0.6)$ and rich $(\phi=1.4)$ methane/air mixtures. It is seen that the impact of compressibility is higher for the rich methane/ 
air, due to a higher flame propagation velocity. Overall, the effect of compressibility is stronger in the cylindrical geometry.
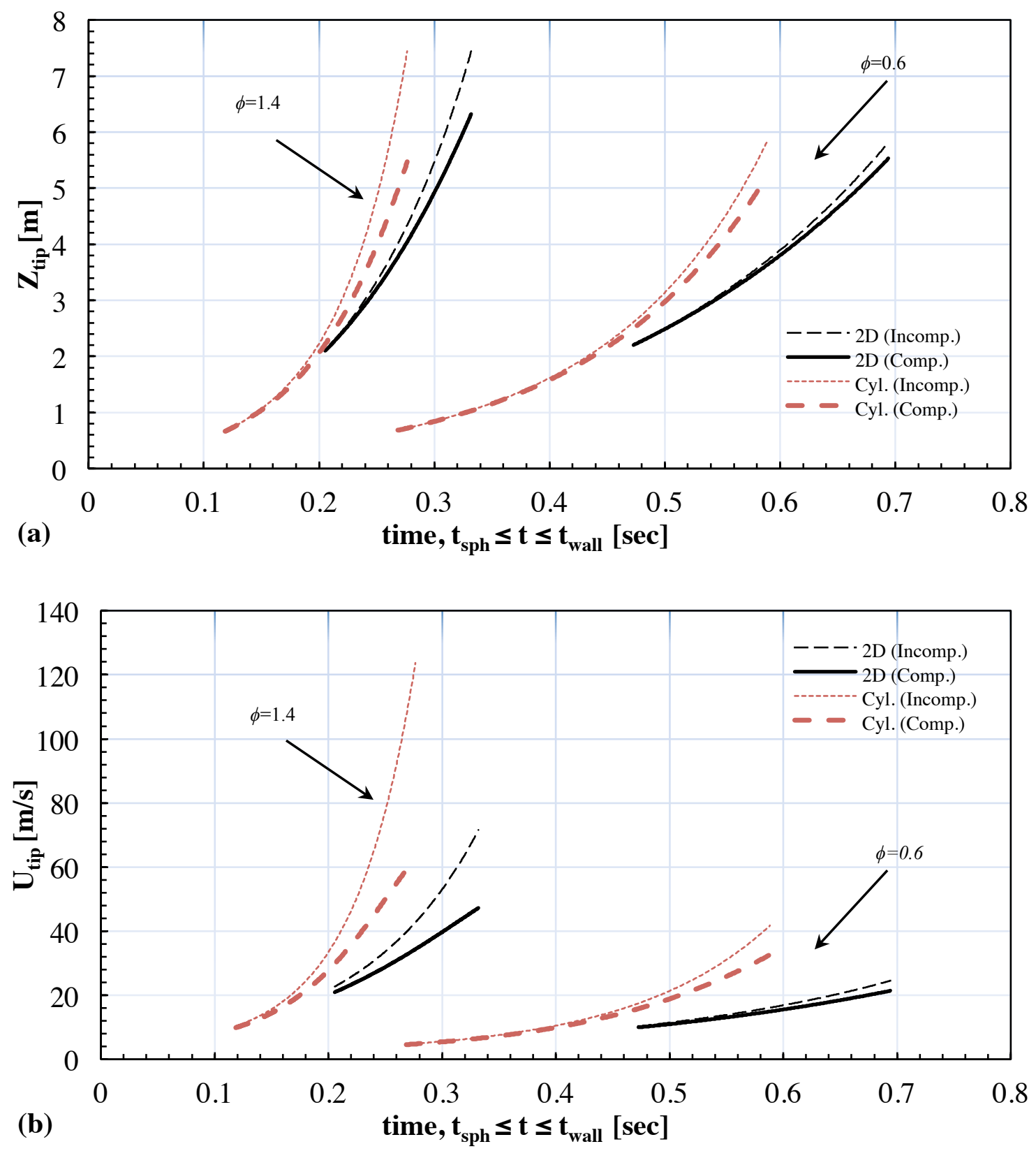

Figure 2.22: Comparison of the incompressible and compressible formulations for the 2D planar and cylindrical geometries: Evolution of the flame tip position $Z_{t i p}$ (a) and its velocity $U_{t i p}$ (b) for the lean $(\phi=0.6)$ and rich $(\phi=1.4)$ methane/air flames. 
The most important conclusion of Figs. 2.18(b) - 2.22(b) is that the flame tip no longer attains the sound speed regardless of a configuration (2D or cylindrical), of a fuel (methane or propane) and composition (lean, stoichiometric or rich) during the stage of finger flame acceleration. It is presumed that other factors such as turbulence can be responsible for the detonation initiation after the termination of "finger" acceleration. As a result, the present study constitutes an important revision on the incompressible flow scenario and qualitatively agrees with the computational and experimental predictions from the literature.

To extend the analysis from gaseous to gaseous-dusty combustion, Figs. 2.23 and 2.24 demonstrate the effect of gas compression on flame acceleration in the presence of combustible dust particles for the 2D-planar and cylindrical geometries, respectively. Namely, the small $\left(r_{s}=10 \mu \mathrm{m}\right)$ and relatively larger $\left(r_{s}=75 \mu \mathrm{m}\right)$ coal particles are considered, with $\phi=0.7$ and $c_{s}=120 \mathrm{~g} / \mathrm{m}^{3}$ in both cases. The event of no particles is also presented. For comparison, the results of the incompressible formulation are also shown. Overall, it is seen that the combustible dust promotes the flame velocity, and the smaller the particles, the stronger acceleration is. According to Fig. 2.23(a), the relative reduction in $Z_{\text {tip }}\left(t_{\text {wall }}\right)$, due to gas compression, is $18 \%$ for $r_{s}=10 \mu \mathrm{m}, 14.4 \%$ for $r_{s}=75 \mu \mathrm{m}$ and $13.6 \%$ for the event of no dust. As for the flame tip velocity, Fig. 2.23(b), the relative reduction in $U_{\text {tip }}\left(t_{\text {wall }}\right)$ constitutes $51 \%$ for $r_{s}=10 \mu \mathrm{m}, 34 \%$ for $r_{s}=75 \mu \mathrm{m}$ and $32 \%$ for no dust. Figure 2.24 is a counterpart of Fig. 2.23 for the cylindricalaxisymmetric geometry. In Fig. 2.24(a), the relative reduction in $Z_{\text {tip }}\left(t_{\text {wall }}\right)$ due to gas compression is $39 \%$ for $r_{s}=10 \mu \mathrm{m}, 27 \%$ for $r_{s}=75 \mu \mathrm{m}$, and $26 \%$ for the event of no dust. As for the flame tip velocity, Fig. 2.24(b), the relative reduction in $U_{\text {tip }}\left(t_{\text {wall }}\right)$ constitutes $68 \%$ for $r_{s}=10 \mu \mathrm{m}, 50 \%$ for $r_{s}=75 \mu \mathrm{m}$ and $48 \%$ for no dust. Consequently, gas compression moderates methane/air/coal-dust flame acceleration, and the effect appears even stronger than that for gaseous combustion. 

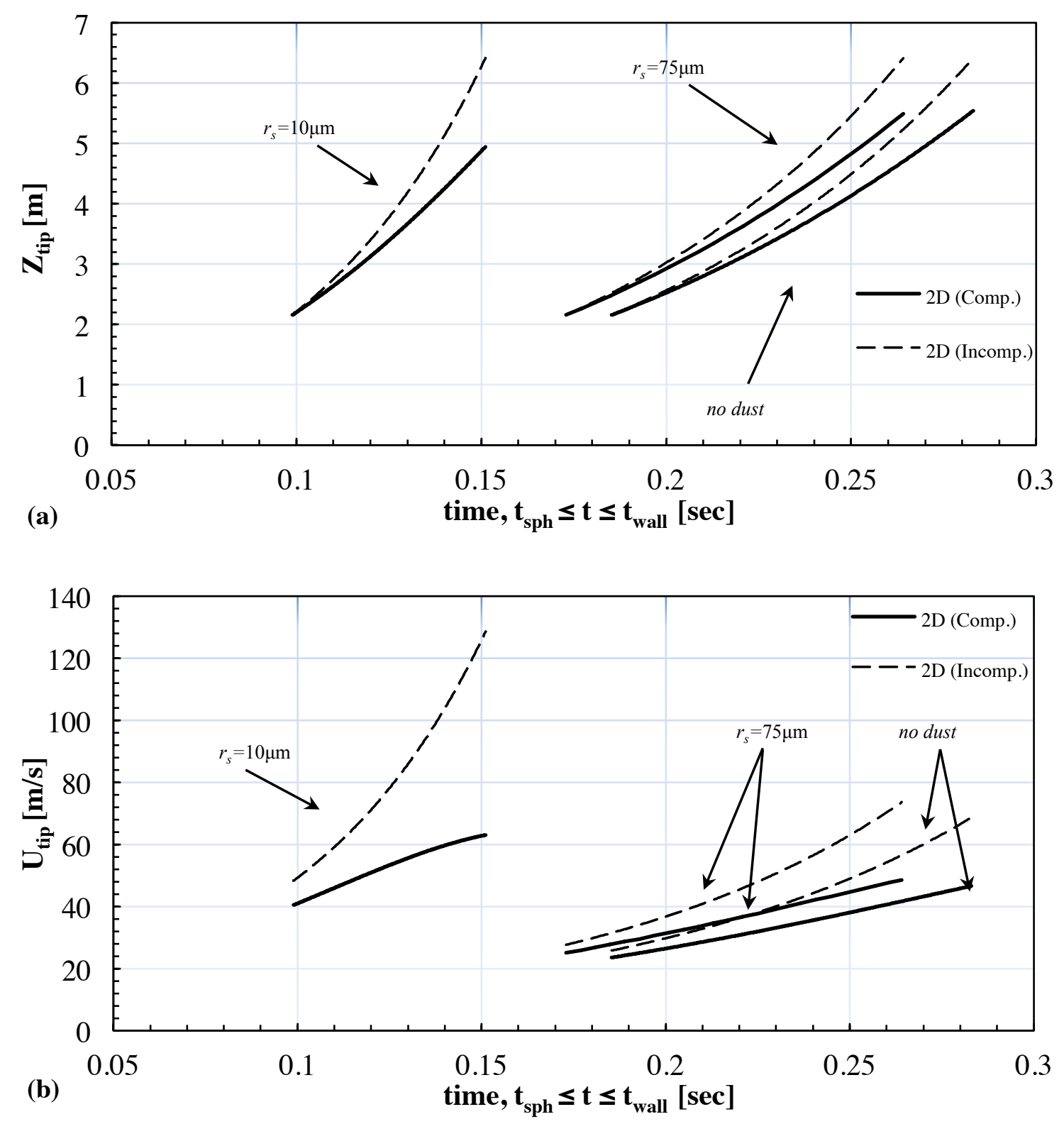

Figure 2.23: Comparison of the incompressible and compressible formulations for the 2Dplanar geometry: Evolution of the flame tip position $Z_{t i p}$ (a) and its velocity $U_{t i p}$ (b) for the methane/air flames of the equivalence ratio $\phi=0.7$ in the presence of combustible dust of the concentration $\mathrm{c}_{\mathrm{s}}=120 \mathrm{~g} / \mathrm{m}^{3}$ and the mean particle radii $r_{s}=10 \mu \mathrm{m} ; 75 \mu \mathrm{m}$. 

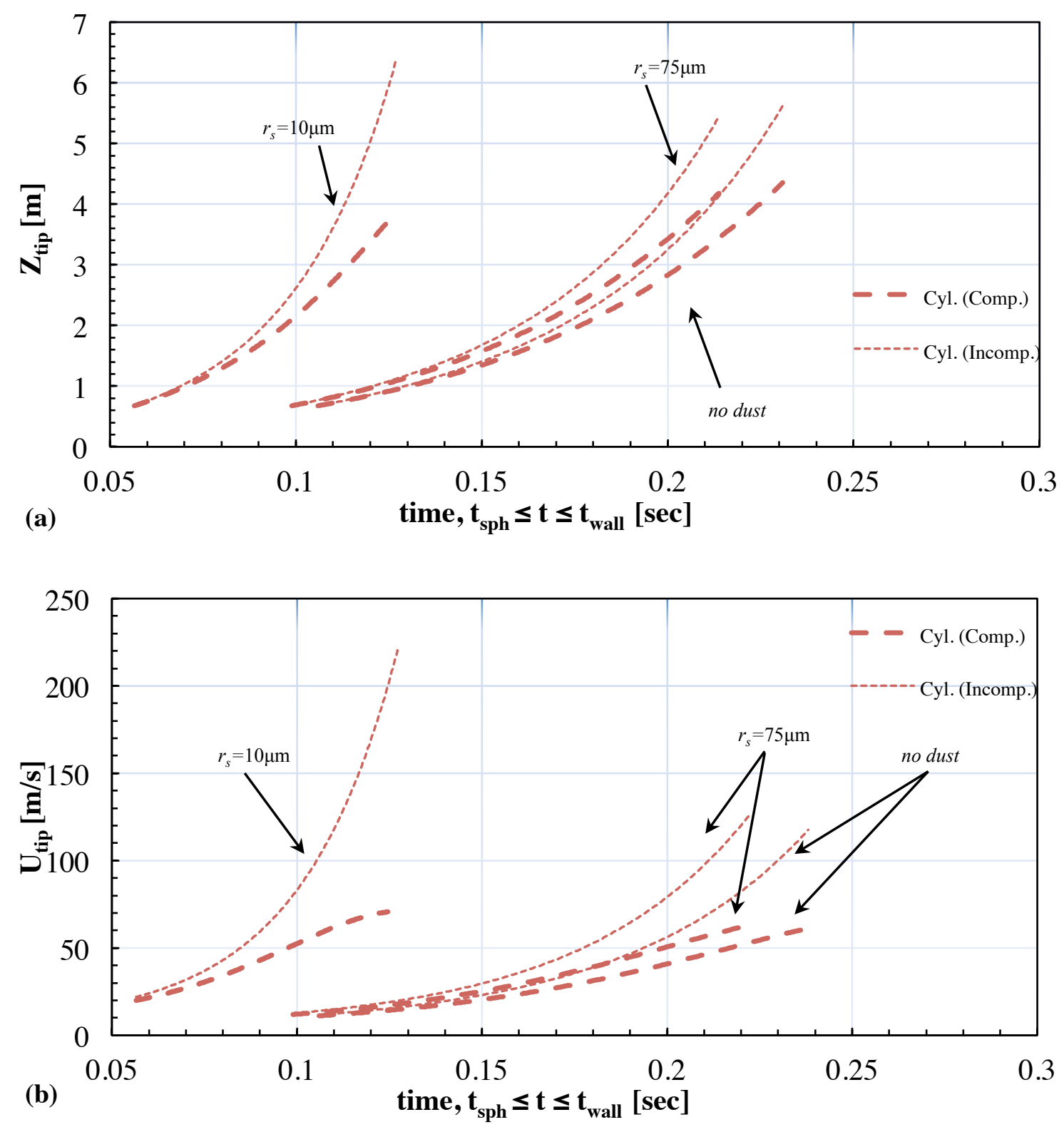

Figure 2.24: Comparison of the incompressible and compressible formulations for cylindrical geometry: Evolution of the flame tip position $Z_{t i p}$ (a) and its velocity $U_{t i p}$ (b) for the methane/air flames of the equivalence ratio $\phi=0.7$ in the presence of combustible dust of the concentration $\mathrm{c}_{\mathrm{s}}=120 \mathrm{~g} / \mathrm{m}^{3}$ and the mean particle radii $r_{s}=10 \mu \mathrm{m} ; 75 \mu \mathrm{m}$.

The maximal flame tip position and velocity attained during finger flame acceleration, i.e. when the flame skirt contacts the passage wall, $Z_{\text {tip }}\left(t_{\text {wall }}\right)$ and $U_{\text {tip }}\left(t_{\text {wall }}\right)$, are presented versus the particle concentration in Fig. 2.25, for $r_{s}=25 \mu \mathrm{m}$ and $\phi=0.7$ in the 2D-planar and cylindrical- 
axisymmetric geometries. Here, Figs. 2.25 (a, b) are devoted to the combustible particles, while the case of inert particles is shown in Figs. 2.25 (c, d). It is readily seen that unlike the particle size, the influence of concentration on the flame dynamics is weaker: the effect is minor for combustible dust, and it is moderate for inert particles.

Finally, the flame evolutions in the situations of no dust, combustible dust, inert dust, and their combination are compared in Figs. 2.26 and 2.27 for the 2D-planar and cylindricalaxisymmetric geometries, respectively, for $\phi=0.7, \mathrm{c}_{\mathrm{s}}=120 \mathrm{~g} / \mathrm{m}^{3}$, and $r_{s}=25 \mu \mathrm{m}$ in both figures. It is observed that the combustible and inert particles provide noticeable deviations from the nodust curves by promoting (combustible dust) or moderating (inert dust) flame acceleration. When both combustible and inert particles are present in the mixture, their impacts oppose each other such that their net effect resembles that of no dust. Still, the relative effect of the combustible dust exceeds that of the inert one. The influence of gas compression is also seen. Quantitatively, in a 2D-planar geometry, Fig. 2.26(a), the relative reduction in $Z_{\text {tip }}\left(t_{\text {wall }}\right)$ due to gas compression is $14 \%$ for no dust, $10 \%$ for the inert dust, $17 \%$ for the combustible dust and $13 \%$ for their combination. As for the associated flame tip velocity, Fig. 2.26(b), here gas compression provides the relative reduction in $U_{\text {tip }}\left(t_{\text {wall }}\right)$ of $32 \%$ for no dust, of $23 \%$ for the inert dust particles, of $39 \%$ for the combustible particles, and of $30 \%$ for their combination. As for the cylindrical-axisymmetric geometry, Fig. $2.27, Z_{\text {tip }}\left(t_{\text {wall }}\right)$ reduces by $25 \%$ for no dust, by $19 \%$ for the inert dust, by $31 \%$ for the combustible dust and by $24 \%$ for their combination, as shown in Fig. 2.27(a). Similarly, $U_{\text {tip }}\left(t_{\text {wall }}\right)$, Fig. 2.27(b), reduces due to gas compression by $48 \%$ for no dust, by $36 \%$ for the inert dust, by $56 \%$ for the combustible dust and by $45 \%$ for their combination. If the mixture composition approaches the stoichiometry, $\phi=1$, then the relative reduction in $Z_{t i p}$ and $U_{t i p}$, due to gas compression, is expected to increase as discussed in the previous section.

Finally, the effect of geometry is shown in Fig. 2.28 for the combustible and inert particles. It is seen that the impact of gas compression is stronger in the cylindrical geometry. Additionally, during the time $t_{\text {wall }}-t_{s p h}$, a flame elongates more strongly in the cylindrical geometry than in the $2 \mathrm{D}$ one, and this range is smaller for the combustible particles due to the ability of fast pyrolysis. 

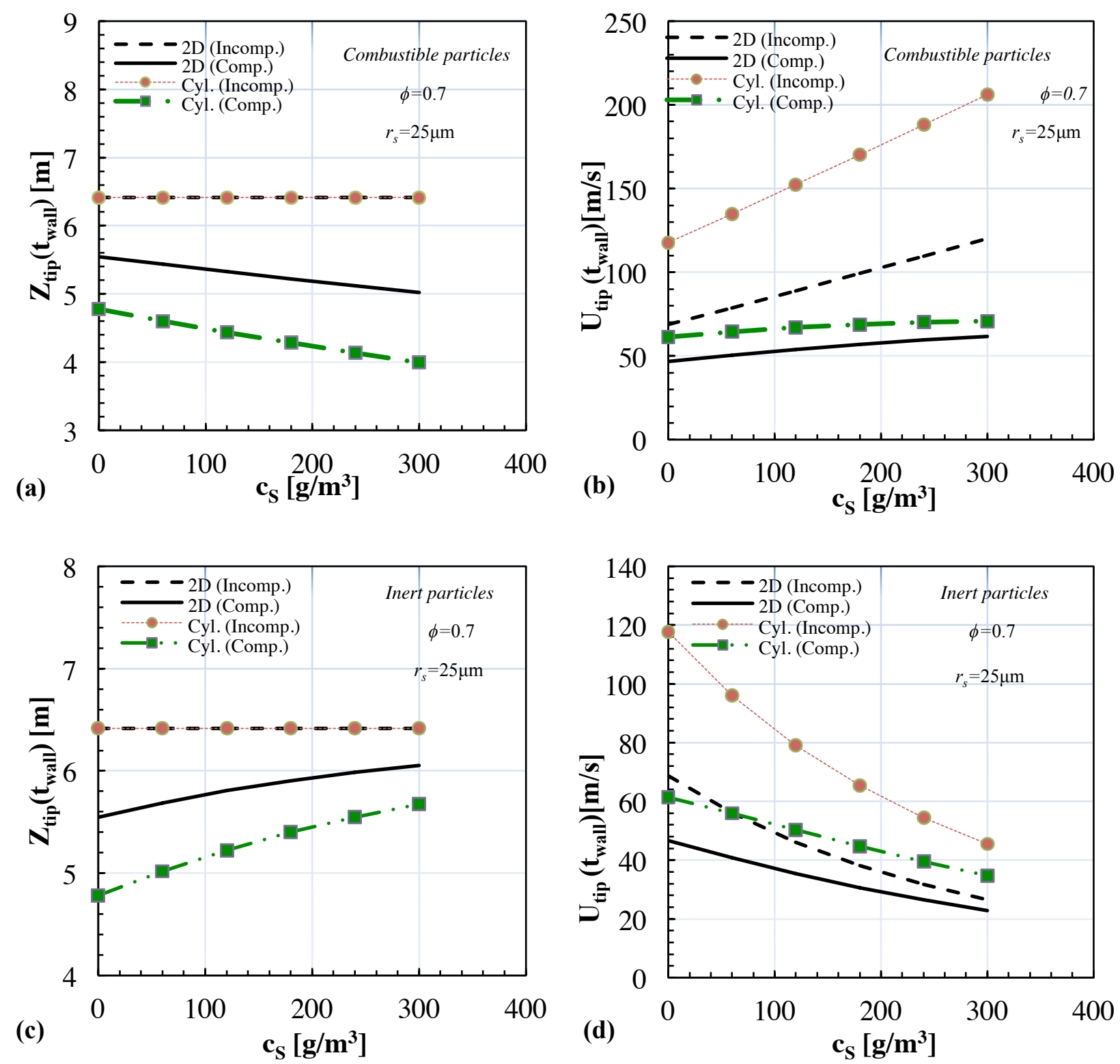

Figure 2.25: Comparison of the incompressible and compressible formulations for the 2Dplanar and cylindrical-axisymmetric geometries: the maximal coordinate $Z_{\text {tip }}\left(t_{\text {wall }}\right)$ (a, c) and velocity $U_{\text {tip }}\left(t_{\text {wall }}\right)(\mathbf{b}, \mathbf{d})$ versus the combustible $(\mathbf{a}, \mathbf{b})$ and inert $(\mathbf{c}, \mathbf{d})$ particle concentration for $\phi=0.7$ and the mean particle radii $r_{s}=25 \mu \mathrm{m}$. 

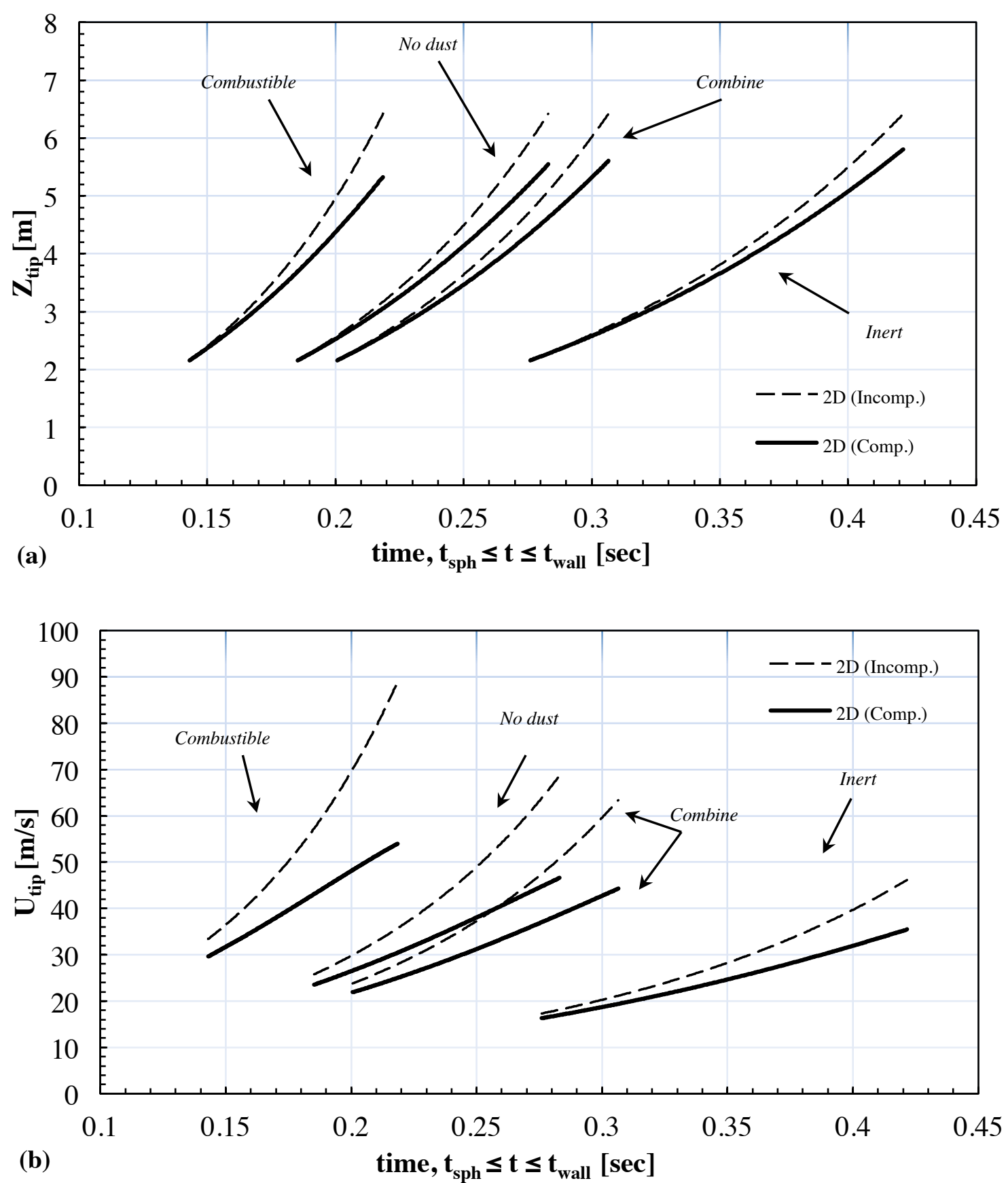

Figure 2.26: Comparison of the incompressible and compressible formulations for the 2Dplanar geometry: Evolution of the flame tip position $Z_{t i p}$ (a) and its velocity $U_{t i p}$ (b) for the methane/air flames of equivalence ratio $\phi=0.7$ in the presence of the combustible, inert and combined particles of the concentration $\mathrm{c}_{\mathrm{s}}=120 \mathrm{~g} / \mathrm{m}^{3}$ and the mean particle radius $r_{s}=25 \mu \mathrm{m}$. 

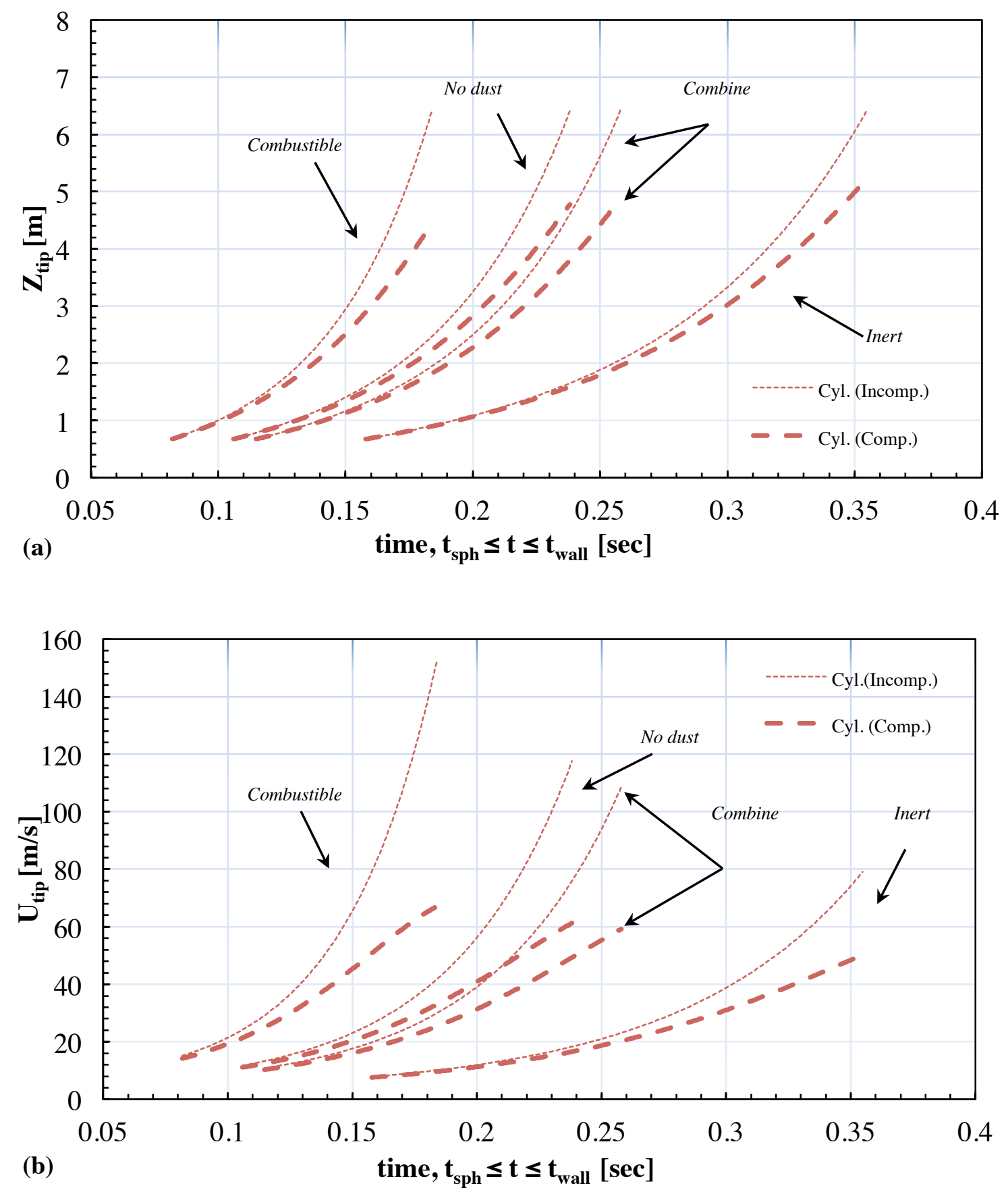

Figure 2.27: Comparison of the incompressible and compressible formulations for the cylindrical-axisymmetric geometry: Evolution of the flame tip position $Z_{t i p}$ (a) and its velocity $U_{t i p}$ (b) for the methane/air flames of the equivalence ratio $\phi=0.7$ in the presence of combustible, inert and combined particles of the concentration $\mathrm{c}_{\mathrm{s}}=120 \mathrm{~g} / \mathrm{m}^{3}$ and the mean particle radius $r_{s}=25 \mu \mathrm{m}$. 

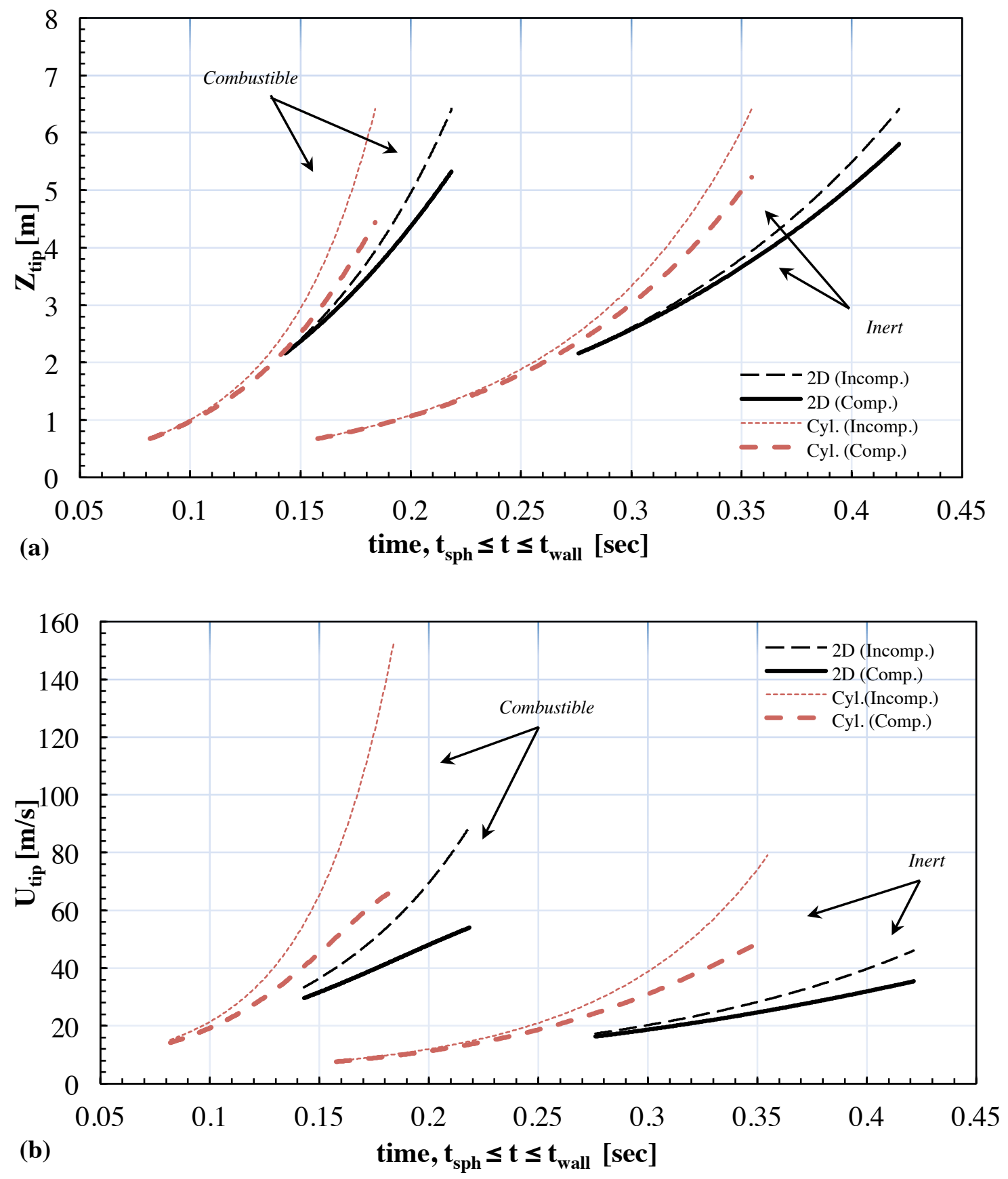

Figure 2.28: Comparison of the incompressible and compressible formulations for the 2Dplanar and cylindrical-axisymmetric geometries: evolution of the flame tip position $Z_{t i p}$ (a) and its velocity $U_{t i p}$ (b) for the methane/air flames of the equivalence ratio $\phi=0.7$ in the presence of the combustible, inert and combined particle of the concentration $\mathrm{c}_{\mathrm{s}}=120 \mathrm{~g} / \mathrm{m}^{3}$ and the mean particle radius $r_{s}=25 \mu \mathrm{m}$. 
Last, it is noted that different motions of dust participles may influence the flame evolution. Moreover, the distribution of the velocities of the particles will lead to a non-uniform distribution of the dust concentration, thereby creating a certain spatial distribution of the local burning properties, such as that of equivalence ratio $\phi_{s}$, laminar flame velocity $S_{d, L}$, etc. In fact, the coal dust distribution is typically non-uniform in coal mines and a stationary dense coal dust layer may spread through the bottom of a passage. In particular, a gaseous-based detonation wave may produce a strong shock that can lift and entrain the dust layer. Over time, the shock weakens, but the shock-heated fluid is ignited by the lifted dust, which initiates a secondary combustion process [73]. Such a lifted dust layer may resemble a linear, cubic, or even parabolic distribution of the dust concentration in space, due to the different energy levels of complex magnetic forces. The impact of these local spatial variations of the burning properties on the global flame propagation scenario will be presented in the following Secs. 3 and 4, and is the topic of papers 3 and 4.

First, the question of local variation of flame speed $S_{L}$ is addressed from a fundamental point of view, by incorporating the spatial functions, including the linear, cubic and parabolic distributions mentioned above, instead of a constant $S_{L}$, specifically in the finger flame acceleration mechanism (Sec. 3). Second, from a practical point of view, propagation of a gaseous-dusty premixed flame front in a channel, resembling a methane/air fire scenario in a coal mine, is studied by means of computational simulations for a certain set of parameters. Specifically, flame acceleration due to wall friction is analyzed for homogenous, linear, cubic and parabolic distributions (Sec. 4). 


\section{Effect of Spatial Variations of the Laminar Flame Speed on the Global Finger-Flame Acceleration Scenario}

The majority of theories associated with the variety of flame acceleration scenarios are based on the "geometrical formulation": namely, the wrinkled-to-planar flame velocities ratio, $U_{w} / S_{L}$, is evaluated as the respective corrugated flame surface area scaled by the planar one, while the entire combustion chemistry is immersed into $S_{L}$. The latter has conventionally been considered as a constant, depending on the thermal-chemical properties of the fuel mixture only, irrespective of the configuration and hydrodynamics $[18,32]$. However, in the practical reality, the value of $S_{L}$ may experience spatial and temporal variations, caused by numerous factors such as heat loss [74], non-uniform distribution of the equivalence ratio [75], pressure and temperature variations $[76,77]$ or, as in coal mines, due to the local variations of the coal dust and/or rock dust impurities $[30,78]$.

In fact, variations of the planar flame speed have been addressed in a number of studies. In particular, Grune et al. [79, 80] analyzed, experimentally, deflagration-to-detonation transitions in stratified hydrogen-air mixtures in a semi-confined geometry with various linear hydrogen concentration gradients. Shi et al. [81] showed the effect of stratification on the laminar flame speed by means of a numerical analysis of the local fuel consumption, burnt gas expansion, as well as the hydrodynamic effects of the flow velocity variations. Hemchandra and Lieuwen [82] demonstrated a significant role of the local spatial variations of the consumption flame speed, along the flame front, in the presence of turbulence. However, none of these works provided guidance on how to combine local and global flame dynamics, and the controlling strategies to prevent or promote flame acceleration need rigorous quantitative assessment of the impacts of local $S_{L}$-variations on the global flame front morphology and propagation speed $U_{w}$.

To address this demand, this section initiates a systematic research how to incorporate local planar flame speed variations into the formulations on the global, corrugated flame velocity in 
the situations when the $S_{L}$-distribution is externally imposed, being a free functional employed in the formulation. The present work is the first step in this direction. Specifically, here we focus on the mechanism of flame acceleration associated with a finger-like shape of the flame front at the initial stages of burning in tubes; the impact of $S_{L}$-variations on the other acceleration mechanisms such as that due to wall friction will be presented in Sec. 4, by means of computational simulations of the compressible hydrodynamic and combustion equations in the presence of combustible coal particles.

The present dissertation extends the finger flame formulation [32] to account for the local variations of the planar flame speed $S_{L}$. Starting with $S_{L}=$ const, the linear, parabolic and hyperbolic spatial $S_{L}$ - distributions are subsequently included into the analysis. Thereby, the theory of flame acceleration due to a finger-like flame shape for the 2D and cylindrical geometries is developed, accounting for the $S_{L}$-variations.

\subsection{Analytical Formulation}

While an analysis of realistic $S_{L}$-distributions occurring in practice would require further details, we start with the simplest distributions allowing for an analytic consideration, and compare them to a situation where $S_{L}=S_{L}^{0}=$ const. In our study, the local flame velocity at the sidewall always exceeds zero, otherwise a flame skirt would never contact the sidewall, which would be unphysical and yield unlimited flame acceleration. Specifically, linear, parabolic and hyperbolic $S_{L}$-distributions in the 2D planar and cylindrical axisymmetric geometries are employed,

$$
S_{L}=S_{L}^{0}(a-\varsigma|r / R|), \quad S_{L}=S_{L}^{0}\left(a-\varsigma(r / R)^{2}\right), \quad S_{L}=S_{L}^{0}\left[\tanh \left(a\left(a-\varsigma(r / R)^{2}\right)\right) / \tanh a\right],
$$

where $S_{L}^{0}$ would be the referenced planar flame speed in the case of uniform $S_{L}$-distribution while $a$ and $\varsigma$ are the free parameters of the formulation. In particular, $a=1$ and $\varsigma=0.8$ are employed to provide strong acceleration near the tube centerline but weak acceleration near the wall, thereby moderating global flame acceleration. In contrast, to promote flame spreading at the sidewall while moderating it at the centerline, $a=0.2, \varsigma=-0.8$ are taken. 
The distributions (3.1) are presented in Fig. 3.1. While a linear distribution is the most encountered distribution in many studies [73, 79, 80], the distributions (3.1) are focused for the following reasons: first being simple from the analytical viewpoint, these distributions can actually be of practical relevance. Second, the planar flame speed depends on the major thermal parameters (such as the Lewis number, Le, thermal conductivity, $k_{u}$, fuel concentration $\chi$, density $\rho$, burnt temperature $T_{b}$, etc. $)$ as $S_{L} \propto \sqrt{f\left(L e, \rho, k_{u}, \chi\right) \exp \left(-E_{a} / R T_{b}\right)}$. Therefore, any linear variation of $L e, \rho, k_{u}$, or $\chi$ (under the squareroot) corresponds to a hyperbolic-like $S_{L^{-}}$ function. Similarly, any local temperture variations in the exponential term can generate a parabolic-like variation of the laminar flame speed. This situation may also justify the physical interpretation of our distribution functions. It should be noted that these distributions do not have the same averaged flame speed across the cross section since the current aim of this study is to analyze the effect of diffirent ditributions.

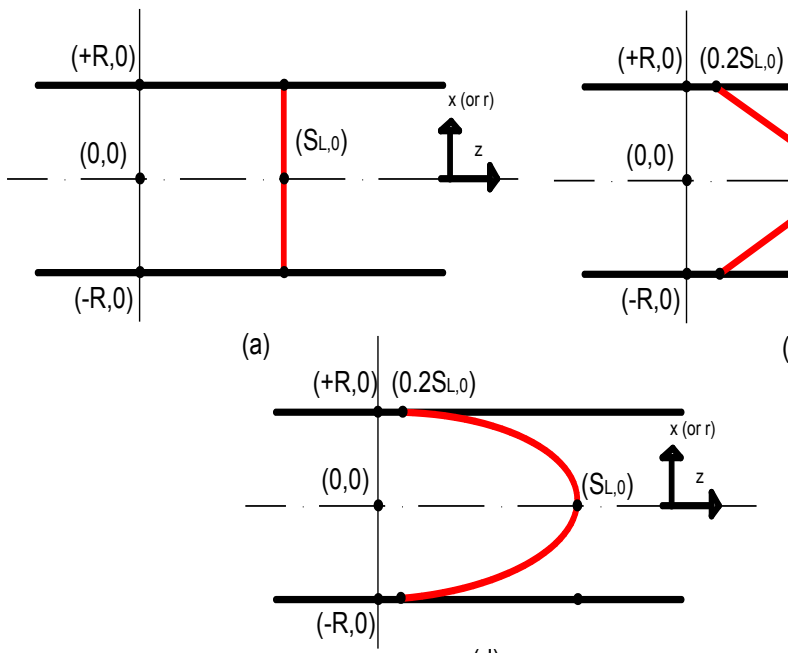

(d)

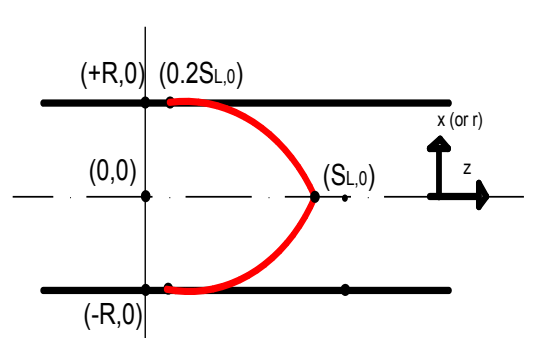

(b)

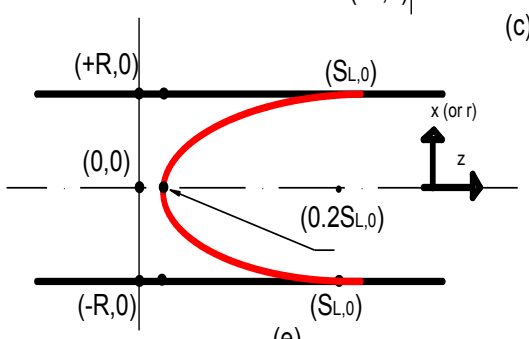

(e)

Figure 3.1: Schematic of various $S_{L}$ - distributions, Eq. (3.1): (a) constant, (b) linear, (c) tangential-hyperbolic, (d) parabolic (promotion along the axis, $a=1, \varsigma=0.8$ ), and (e) parabolic (promotion through wall, $a=0.2, \varsigma=-0.8$ ).

A flame propagating in a $2 \mathrm{D}$ channel or a cylindrical tube of radius (half-width) $R$ is considered with slip adiabatic walls and with one end closed, as illustrated in Fig. 2.1 (see Sec. 2.1). The ignition point is at the closed end at the symmetry axis (centerline). Next, the $S_{L}$ dependences, Eq. (3.1), are incorporated into the theory of finger flame acceleration for 2D and 
axisymmetric cylindrical cases. Although some analytical formulations of finger flame acceleration mechanism were presented in the Sec. 2, for the convenience of the reader, a few of are also repeated here.

\subsubsection{D Planar Geometry}

To develop a 2D finger flame formulation, the incompressible continuity equation is considered:

$$
\partial u_{x} / \partial x+\partial u_{z} / \partial z=0
$$

with the boundary conditions $\left.u_{z}\right|_{z=0}=0,\left.u_{x}\right|_{x=x_{f}}=0$. A conventional (Landau) approach of an infinitesimally thin flame front is adopted. A similar coordinate system is used for the analyses as illustrated in Fig. 2.4. Assuming a potential flow in the fuel mixture (label "1"), one can find the flow velocity components from continuity equation (3.2):

$$
u_{z, 1}=A_{1}(t) z, \quad u_{x, 1}=A_{1}(t)(R-x),
$$

The counterpart of Eq. (3.3) in the burnt matter reads

$$
u_{z, 2}=A_{2}(t) z, \quad u_{x, 2}=-A_{2}(t) x .
$$

It is noted that while the flow is generally rotational in the burnt matter (with singularity occurring), due to a curved flame shape, this flow can be treated as potential close to the endwall, where the flame front is locally planar. Hence, the baroclinic effects due to the local perturbation can be neglected in that region. The matching conditions at the flame front are

$$
d x_{f} / d t-u_{x, 1}=S_{L}\left(x_{f}\right), \quad u_{x, 1}-u_{x, 2}=(\Theta-1) S_{L}\left(x_{f}\right), \quad u_{z, 1}=u_{z, 2} .
$$

Here, the $1^{\text {st }}$ equation specifies a locally-variable flame propagation speed with respect to the fuel mixture, the $2^{\text {nd }}$ describes the jump of the normal velocity, and the $3^{\text {rd }}$ shows the continuity of the tangential velocity. Together, Eqs. (3.3) - (3.5) yield $A_{1}=A_{2}=(\Theta-1) S_{L}\left(x_{f}(t)\right) / R$ such that the evolution equation for the flame skirt position, $x_{f}$, reads:

$$
\frac{d x_{f}}{d t}=S_{L}\left(x_{f}\right)\left(\Theta-\frac{\Theta-1) x_{f}}{R}\right) .
$$

Similarly, one can also find the evolution equation for the flame tip position, $Z_{t i p}$, by considering the flow along the channel centerline, $x=0$, similar to Eq. (3.6), and with Eq. (3.4) for $u_{z, 2}$ :

$$
\frac{d Z_{t i p}}{d t}-u_{z, 2}=\frac{d Z_{t i p}}{d t}-(\Theta-1) S_{L}\left(x_{f}\right) \frac{Z_{t i p}}{R}=\Theta S_{L}^{0} .
$$


In fact, Eq. (3.7) describes propagation of the flame tip with respect to the burnt matter. It is noted that this equation is applicable as long as the flame tip is at the centerline, such that the flame front is locally planar therein and the flow in the burnt matter can be treated as a potential flow. However, this is not the case if the tip is far from the centerline, because flow is rotational in the mean that event.

\subsubsection{Cylindrical Axisymmetric Geometry}

Here, the 2D formulation above is reproduced for the axisymmetric geometry. The cylindrical axisymmetric continuity equation for the incompressible flow reads [32]

$$
\frac{\partial\left(r u_{r}\right)}{(r \partial r)}+\frac{\partial u_{z}}{\partial z}=0
$$

with the boundary conditions $\left.u_{z}\right|_{z=0}=0,\left.u_{r}\right|_{r=r_{f}}=0$. Similar to the $2 \mathrm{D}$ case, assuming a potential flow in the fuel mixture, the axial and radial flow velocity component can be found from Eq. (3.8) as:

$$
u_{z, 1}=A_{1}(t) z, \quad u_{r, 1}=\frac{A_{1}(t)}{2\left(\left(R^{2}-r^{2}\right) r^{-1}\right)} .
$$

Their counterparts in the burnt matter are

$$
u_{r, 2}=\frac{-A_{2}(t) r}{2}, \quad u_{z, 2}=A_{2}(t) z
$$

and the matching conditions are the same as in $2 \mathrm{D}$, Eq. (3.5). Then $A_{1}=A_{2}=2(\Theta-1) S_{L}\left(r_{f}\right) r_{f} / R^{2}$ such that the flame "skirt" evolution equation reads

$$
\frac{d r_{f}}{d t}=S_{L}\left(r_{f}\right)\left(\Theta-\frac{(\Theta-1) r_{f}^{2}}{R^{2}}\right) \text {. }
$$

It is noted that $r_{f}=r_{f}(t)$. One can also find the evolution equation for the flame tip by considering the flow along the centerline, $r=0$, similar to the $2 \mathrm{D}$ case, where the flame front is locally planar and flow in the burnt matter is a potential flow,

$$
\frac{d Z_{t i p}}{d t}-\frac{2(\Theta-1) S_{L}\left(r_{f}\right) r_{f}}{R^{2}} Z_{t i p}=\Theta S_{L}^{0}
$$




\subsection{Results and Discussion}

Next, various spatial dependences of the laminar burning velocity are incorporated into the final evolution equation of the flame skirt and tip, namely, $S_{L}\left(x_{f}\right)$ into Eqs. (3.6) and (3.7) in a 2D geometry and $S_{L}\left(r_{f}\right)$ into Eqns. (3.11) and (3.12) in a cylindrical-axisymmetric configuration. In each geometry, the final set of equations has been solved numerically by using a $4^{\text {th }}$-order Runge-Kutta iterative method, with $\Theta=8$, typical for hydrocarbon flames [2, 17], employed in all cases.

Figure 3.2 presents the time evolution of the scaled flame skirt position, $x_{f} / R$, Fig. 3.2(a), and the scaled flame tip position, $Z_{\text {tip }} / R$, Fig. 3.2(b), as well as the scaled flame tip velocity, $U_{t i p} / S_{L}$, Fig. 3.2(c), and its scaled acceleration $a_{t i p} R /\left(S_{L}^{0}\right)^{2}$, Fig. 3.2(d) in a 2D planar geometry, with various $S_{L}$-distributions compared in each plot. Here $U_{t i p}=\dot{Z}_{t i p}, a_{t i p}=\ddot{Z}_{t i p}$, and the scaled time is given by $\tau=t \times S_{L}^{0} / R$. According to Fig. 3.2(a), the flame skirt will reach the wall in the promptest manner for the constant velocity distribution, $S_{L}=S_{L}^{0}=$ const, as compared to other distributions; followed by the hyperbolic, parabolic and liner velocity distributions, respectively. This is also true for the evolution of the flame tip velocity shown in Fig. 3.2(c). The finger-flame acceleration mechanism stops when the flame skirt contacts the wall, $x_{f} / R=1$. The maximum flame tip position at this time, among various $S_{L}$-distributions, is found for the linear distribution (simply because the process takes the longest time in that case); followed by the parabolic, hyperbolic and constant $S_{L}$-distributions, respectively. The largest flame tip velocity in Fig. 3.2(c) is observed for the linear case followed by hyperbolic and parabolic cases, respectively. Figure 3.2(d) shows the evidence of strong acceleration for all considered functions. Indeed, for a typical hydrocarbon flame [2] this flame tip acceleration exceeds gravity acceleration by a factor of $\sim 10^{3}$. 

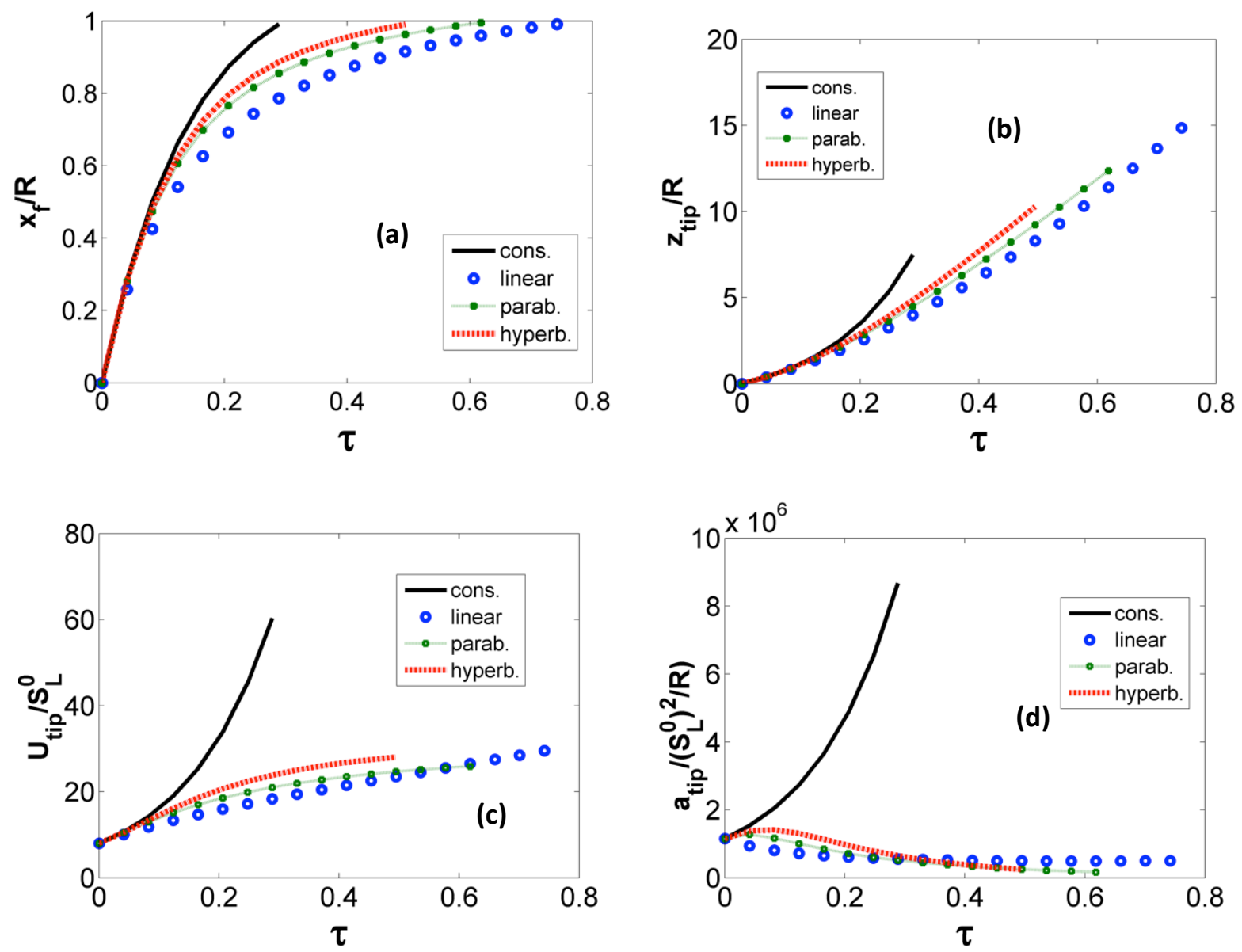

Figure 3.2: $2 D$ geometry: Evolution of the scaled flame skirt position (a), flame tip position (b), flame tip velocity (c), and flame tip acceleration (d) versus scaled time, $\tau=t \times S_{L}^{0} / R$, for the constant, liner, hyperbolic and parabolic $S_{L}$ - distributions.

Figure 3.3 is a counterpart of Fig. 3.2 for the cylindrical-axisymmetric geometry. It is seen that the $2 \mathrm{D}$ and axisymmetric results qualitatively agree, but they differ quantitatively providing larger quantities for the latter case, which is consistent with the results of Sec. 2.2. Indeed, according to Fig. 3.3(a), the flame skirt contacts the wall in the fastest way for the constant velocity distribution, followed by the hyperbolic, parabolic and linear velocity distributions. Again, the flame acceleration mechanism stops when $r_{f} / R=1$. 

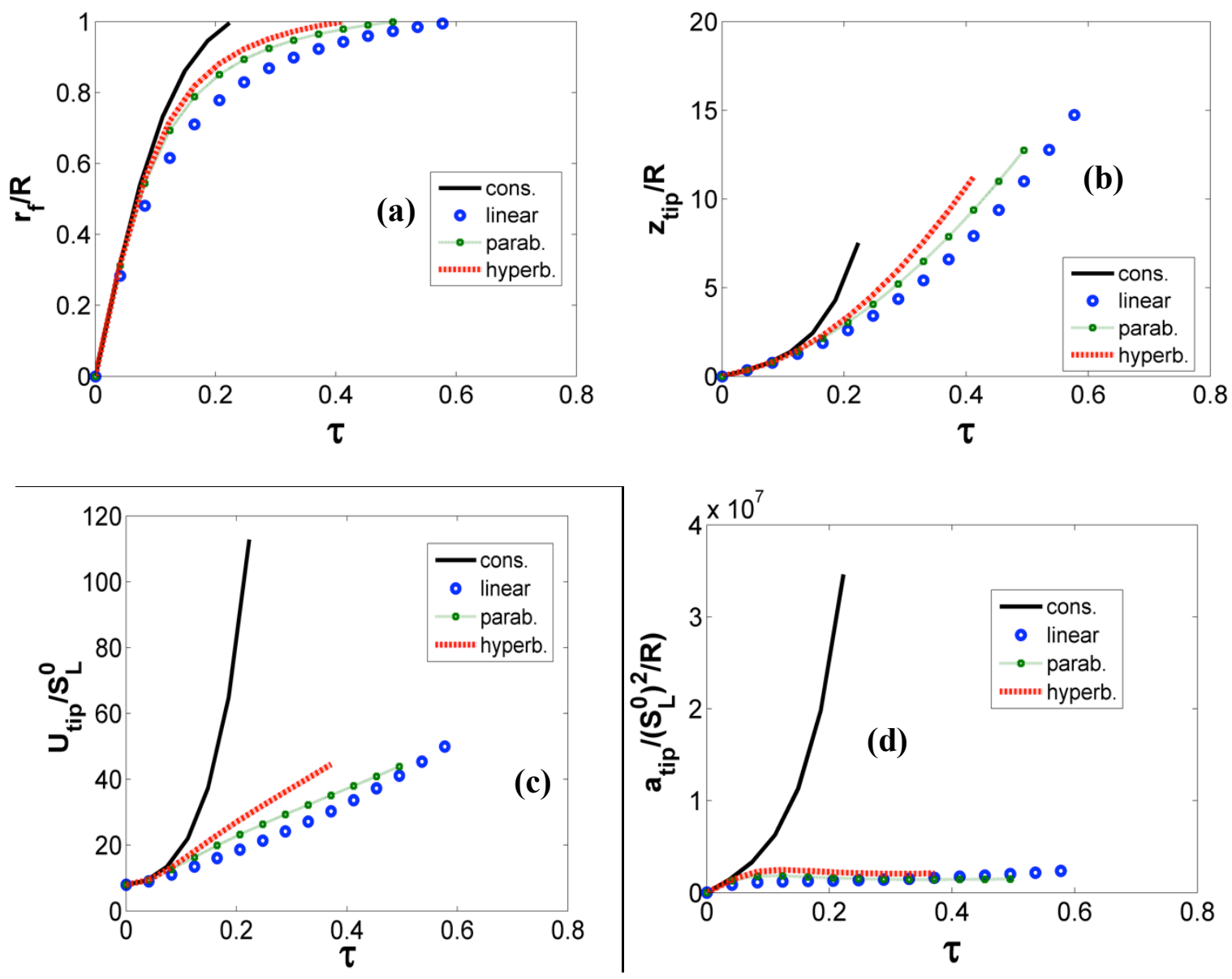

Figure 3.3: Axisymmetric geometry: Evolution of the flame skirt position (a), flame tip position (b), flame tip velocity (c), and flame tip acceleration (d) versus scaled time, $\tau=t \times S_{L}^{0} / R$, for the constant, liner, hyperbolic and parabolic $S_{L}$ - distributions.

One of the key observations is moderation of the acceleration regime for the non-uniform $S_{L}$ -distributions as compared to the $S_{L}=S_{L}^{0}=$ const case. Indeed, while exponential flame acceleration for constant $S_{L}$ in both 2D and cylindrical geometries is observed, the acceleration trend moderates to a linear one for non-uniform $S_{L}$. It is also noted that the maximum scaled flame tip velocity is observed in the cylindrical geometry for constant velocity distribution, and it is $\sim 115 S_{L}$, which still obeys the approach of an incompressible flow for typical hydrocarbon flames (indeed, the speed of sound to the laminar burning velocity ratio, $c_{0} / S_{L} \sim 10^{3}$ for such flames). However, for highly reactive mixtures, such as hydrogen- and/or ethylene-oxygen 
flames, the respective flame tip velocity would approach the speed of sound, as such applying compressible flow characteristics would be imperative [57].

Overall, it is seen from Figs. 3.2 and 3.3 that the evolutions of the flame shape, location and velocity differ for various functions describing the spatial $S_{L}$-distributions. Consequently, by manipulating and imposing these functions, one can potentially be able to control the flame propagation mechanisms. It is noted again that, among the $S_{L}$-distributions considered in this work, the uniform distribution provides the fastest flame acceleration followed by the hyperbolic distribution. The latter has the largest elongation through the upper and lower branch of the flame; see Fig. 3.1(c). As a result, the flame surface area increases more than that for other nonuniform $S_{L}$-distributions, thereby enhancing flame propagation.

To better understand the effect of geometry, both the 2D and axisymmetric configurations are compared in Fig. 3.4, with the flame tip positions and velocities depicted in Fig. 3.4(a) and Fig. 3.4(b), respectively. Here, the constant and parabolic $S_{L}$-distributions are employed in both cases. It is seen that flames propagate/accelerate faster in the cylindrical-axisymmetric geometry as compared to a 2D-planar configuration. For the parabolic $S_{L}$-distribution, the choice of geometry influences the scaled flame tip velocity significantly, indeed, for the cylindrical geometry is almost two times higher than in the $2 \mathrm{D}$ one. This result agrees well with Sec.2.2.
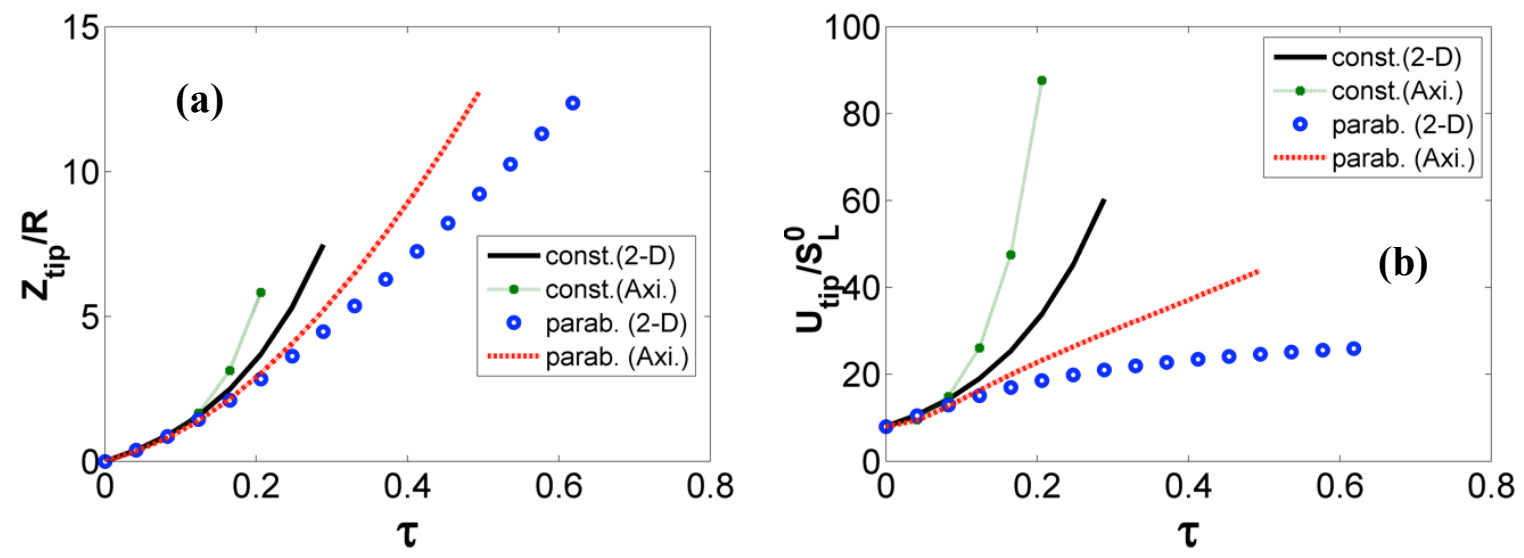

Figure 3.4: Comparison of the 2D-planar and cylindrical axisymmetric cases for the constant and parabolic velocity distributions: Evolution of the flame tip position (a) and its velocity (b) versus scaled time, $\tau=t \times S_{L}^{0} / R$. 
In fact, various $S_{L}$-distributions with various selected free parameters $a$ and $\varsigma$ can either promote or moderate flame acceleration scenario as discussed earlier. All the distributions of Eq. (3.1) moderate flame acceleration as compared to the $S_{L}=S_{L}^{0}=$ const case. Indeed, they provide stronger acceleration through the axis, but weaker acceleration through the wall and, as a result, moderate the global flame dynamics. This is oftentimes the case in the practical reality, for instance, in the pipe-like combustors exhibiting heat losses through their walls and thereby providing slower flame spreading along the wall, but with a higher temperature and flame speed through the centerline. However, flame acceleration can potentially be weaker through the axis, with the promotion through the wall segments, say, as it is oftentimes observed in coal mines. Remember, in that case, there are combustible coal dust particles, distributed along the tunnel wall, which can promote flame acceleration nearby the wall region (at least for certain ranges of dust particles sizes and concentrations). In order to consider the promotion of flame propagation through the wall and its moderation through the centerline, a parabolic function of Eq. (3.1) can be modified as

$$
S_{L}^{*}=S_{L}^{0}\left(0.2+\varsigma(x / R)^{2}\right)
$$

In particular, $\varsigma=0.8$ is employed. In that case, the flame exhibits the earlier defined reference speed $S_{L}^{0}$ at the wall and $0.2 S_{L}$ at the axis, which are exactly opposite to Eq. (3.1). Again, it is noted that these $a$ and $\varsigma$ are free parameters of problem and were chosen arbitrarily. The parabolic and constant $S_{L}$-distributions in that case are compared in Fig. 3.5 for $Z_{\text {tip }}$ (a) and $U_{\text {tip }}$, (b). Based on Fig. 3.5, one arrives to the following physical interpretation: when Eq. (3.1) (yielding the promotion of flame propagation through the centerline and its moderation through walls) is employed, the larger flame surface area is observed through the wall segments, but the local flame speed is lower through the upper and lower sthe ide walls. On the other hand, Eq. (3.13) is used again, promoting the flame spreading through the wall and moderating it through the centerline. While the larger surface area is observed through the wall region, this time the local flame speed is higher through the wall regions as well. Since the burning rate is proportional to flame surface area, the highest flame tip velocity is obtained for $S_{L}^{*}$ of Eq. (3.13), even the same (parabolic) type is employed with the same magnitudes but locally opposite. 

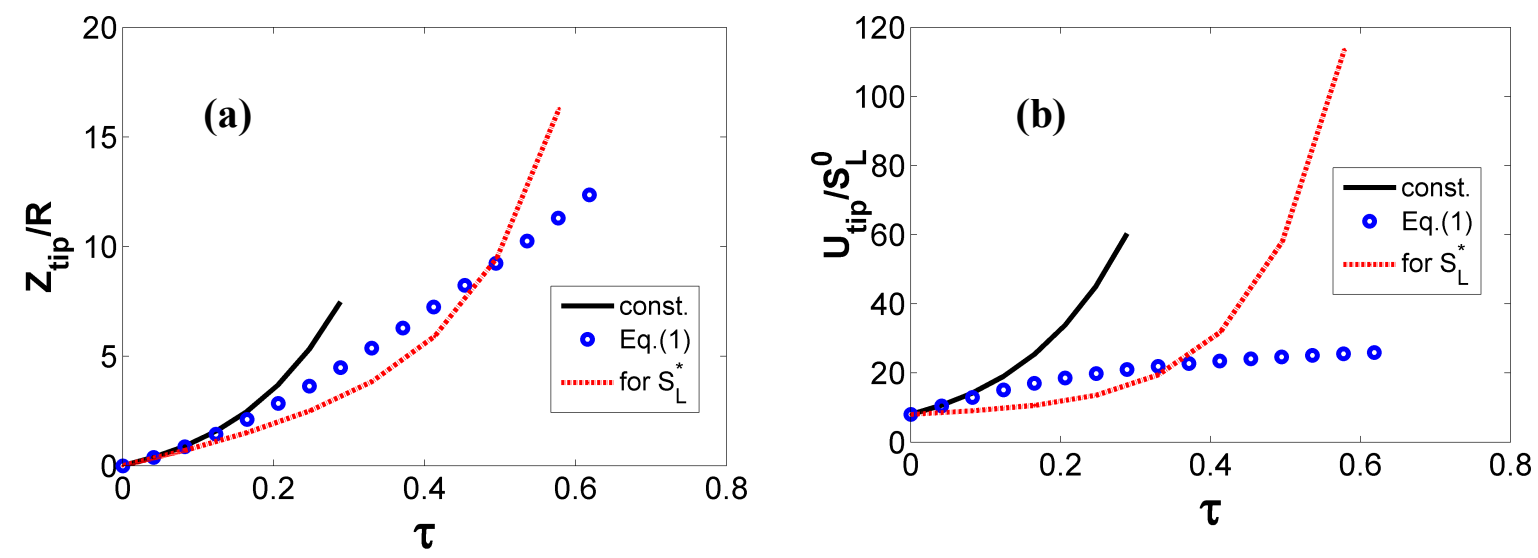

Figure 3.5: 2D geometry: Evolution of the flame tip position (a) and velocity (b) versus scaled time, $\tau=t \times S_{L}^{0} / R$.

A relevant practical application to consider is the risk of fire in a coal mine with a dusty atmosphere. A detailed analytical predictive mining fire scenario was presented in Sec. 2 by assuming a homogeneous distribution of the coal dust particles. To account for the combustible and/or inert dust particles in a global flame-spreading scenario, the Seshadri formulation [30] can be employed for the spatial variation of the flame velocity. Various non-uniform dust distribution gradients can be employed into the concentration term, $n_{s}$, such as linear, hyperbolic, parabolic and other functions of the radial coordinate of a mining passages, $n_{s}(x)$. This situation will be studied in the next section by means of computational simulations of the compressible hydrodynamic and combustion equations for a particular set of parameters as a more specific demonstration of local variation of flame speed. 


\section{Computational Study of Gaseous - Dusty Combustion with Various Dust Distributions}

There is a serious demand from industries dealing with explosive materials for a better understanding of the various scenarios and mechanisms which lead to accidental explosions. In the case of coal mine industry, which globally has one of the highest injury and fatality rates, accidental explosions of flammable gases due to the presence of the combustible dust impurities may result in injuries and deaths of personnel, as well as the destruction of expensive equipment. With this issue in mind, in Sec. 2 a novel predictive scenario of a burning accident in a mining passage has been developed.

While combustion of gaseous fuels and combustible dust particles have been studied reasonably well, see Sec. 2 for a detailed literature review, flame propagation in a combined gaseous-dusty environment, especially with a non-uniform dust distribution in a gas, remains an enigma that commands both fundamental and practical interests. This section focuses on the effects of $S_{L}$-variations on flame acceleration due to wall friction in a gaseous-dusty mixture. Specifically, the computational simulations of the reacting flow equations, with a fullycompressible hydrodynamics and Arrhenius chemistry are performed, with the combustible coal dust particles incorporated into the original (gaseous) computational platform by means of Eq. (2.44). Namely, a real gaseous-dusty environment is replaced by an "effective fluid" with the locally-modified, dust-induced flow and flame parameters. Keeping a coal mine passage in mind, flame propagation in a channel with a large aspect ratio is considered. Here, various spatial distributions of the coal dust concentration are studied, namely: (a) homogenous, (b) linear, (c) cubic and (d) parabolic. While a homogenous dust distribution simply provides a scaling factor as compared to purely gaseous combustion [17], the non-uniform distributions are anticipated to provide qualitatively new features. This section investigates how the dust distribution in a coalmining passage may influence the flame evolution, flame shape, and propagation velocity. The parametric study includes a variety of gradient forms for the dust distribution. It is identified whether a particular dust distribution facilitates or inhibits the flame acceleration process. 
One step of the present thesis is the initial development a Dust-Gas Explosion Model (DGEM) - a computational platform capable of quantifying mining fire hazards, namely, the probability of spontaneous ignition, the evolution of a flame front, and the likelihood of a deflagration-to-detonation transition (DDT). Most CFD explosion models, such as ANSYSFluent and STAR-CCM, are fairly expensive, and do not permit the viewing or modification of the source code [83]. Some noticeable codes that have been used in gas-dust combustion studies are COBRA [84], EXSIM [85], FLACS [86], REAGAS [87], and FLARE [88], which utilize empirical correlations between laminar and turbulent burning velocities. Recently, a code capable of modeling dust explosions has been developed by GexCon called DESC [89]. However, the code is still in its experimental stages and has not been validated extensively. Moreover, although the code claims to solve for dust explosions, it utilizes a turbulent burning velocity correlation from Abdel-Gayed et al. [90] which was developed only for gas explosions. Switching from a homogeneous to a gaseous-dusty environment may substantially modify (or even break) the very empirical dependence, and thereby requires a qualitatively new consideration.

It should also be noted that there are considerable discrepancies between the flame formulations based on the empirical considerations used in existing CFD codes, and formulations obtained from the fundamental principles [91-93]. The latter oftentimes disagrees with the experiments because of some realistic phenomenon omitted in the considerations. Our goal is to bridge the gap by accounting for a realistic effect of local variation of the coal dust distributions on the morphology and dynamics of flame acceleration from both a fundamental and practical point of view.

\subsection{Flame Acceleration due to Wall Friction (a.k.a the Shelkin Mechanism)}

One of the well-known mechanisms of flame acceleration is that by Shelkin [1], which associates flame acceleration with nonslip boundary conditions at the walls. This mechanism is illustrated in Fig. 4.1. Specifically, as a flame front propagates from a closed tube/channel end, the burning matter expands pushing a flow of the fresh fuel mixture. Friction at the pipe walls makes the flow non-uniform, which bends the flame front, and increases its velocity thus 
yielding the associated acceleration. In this acceleration mechanism, the flow is assumed to be laminar and turbulence plays only a supplementary role. Acceleration is unlimited in time, and may continue until detonation initiation.
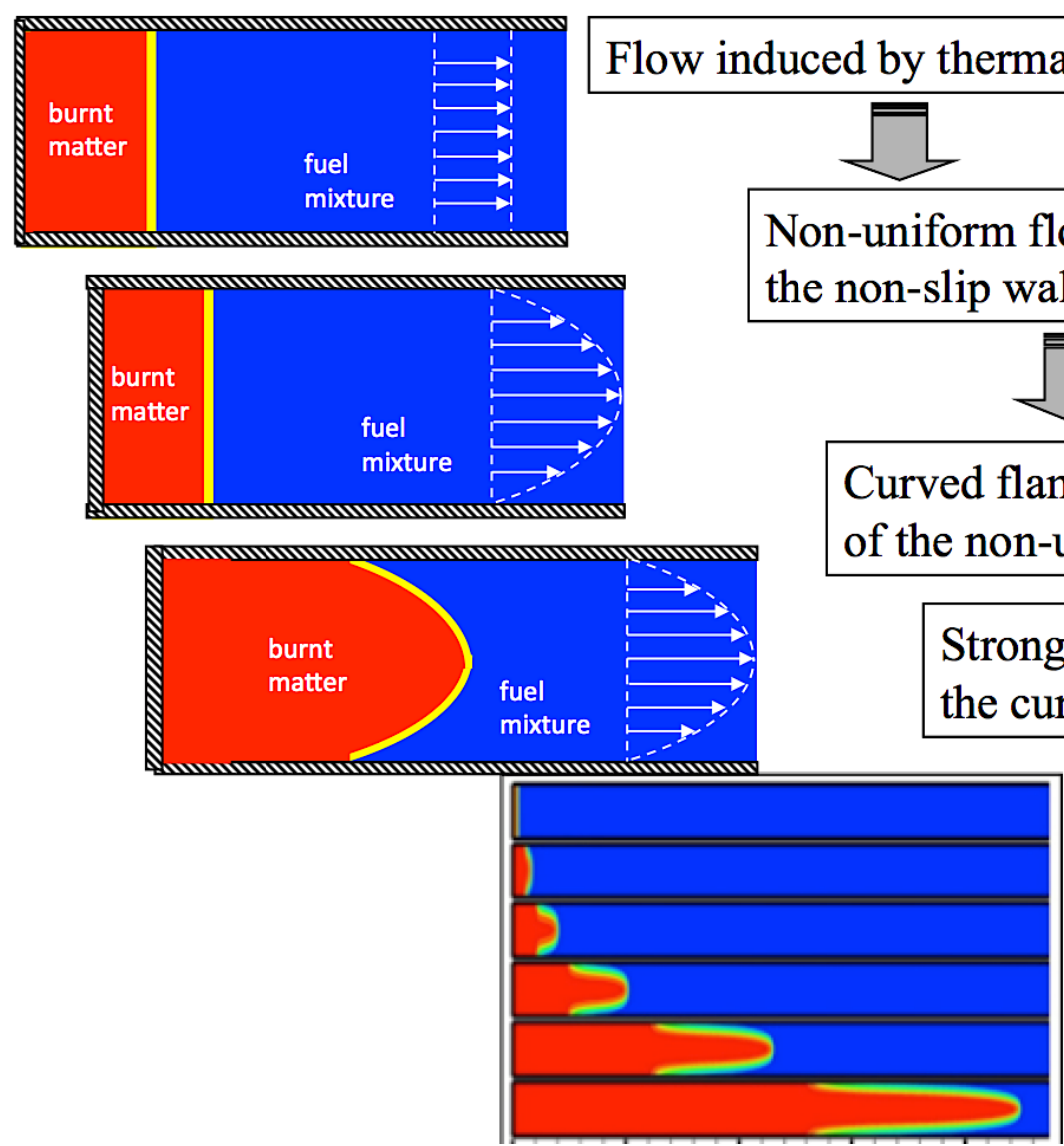

\section{Flow induced by thermal expansion}

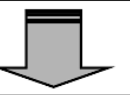

Non-uniform flow because of the non-slip walls

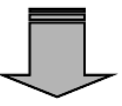

Curved flamefront because of the non-uniform flow

Stronger flow because of the curved flame

Figure 4.1: Flame acceleration due wall friction (a.k.a the Shelkin mechanism).

The Shelkin idea was subsequently developed and quantified by the analytical formulation and extensive computational simulations of Bychkov et al. [17] for 2D channels. Later on, Akkerman et al. [18] extended the analyses for cylindrical tubes. The work was able to predict an initially exponential state of flame acceleration, flame shape and, the velocity profile of the generated mixture flow.

\subsection{The Computational Platform}

In this section, the computational platform is described. An Eulerian computational platform is used, which core was originally developed by Lars-Erik Eriksson at Volvo Aero and consists 
of a fully-compressible finite-volume Navier-Stokes code solving for hydrodynamics and combustion equations in a gaseous environment. The code is based on cell-centered finite volume scheme. It is the $2^{\text {nd }}$-order accurate in time, $4^{\text {th }}$-order accurate in space for the convective terms, and $2^{\text {nd }}$-order accurate in space for the diffusive terms. The platform is adapted to parallel computations (Open MPI) and has been successfully validated on numerous complex reactingflow and aero-acoustic problems $[7,11,17,58]$. The validation of the numerical approach has been performed and compared with exact solutions of flame dynamics [94]. More details about the computational platform can be found in the Ref. [95].

\subsubsection{The Governing Conservation Equations}

The basic governing equations read

$$
\begin{aligned}
& \frac{\partial}{\partial t} \rho+\frac{\partial}{\partial x_{i}}\left(\rho u_{i}\right)=0, \\
& \frac{\partial}{\partial t}\left(\rho u_{i}\right)+\frac{\partial}{\partial x_{j}}\left(\rho u_{i} u_{j}+\delta_{i, j} P\right)-\gamma_{i, j}=0 \\
& \frac{\partial}{\partial t}\left(\rho \varepsilon+\frac{1}{2} \rho u_{i} u_{j}\right)+\frac{\partial}{\partial x_{i}}\left(\rho u_{i} h+\frac{1}{2} \rho u_{i} u_{j} u_{j}+q_{i}-u_{j} \gamma_{i, j}\right)=0 \\
& \frac{\partial}{\partial t}(\rho Y)+\frac{\partial}{\partial x_{i}}\left(\rho u_{i} Y-\frac{\zeta}{\operatorname{Sc}} \frac{\partial Y}{\partial x_{i}}\right)=-\frac{\rho Y}{\tau_{R}} \exp \left(-E_{a} / R_{u} T\right)
\end{aligned}
$$

where $Y$ is the mass fraction of the fuel mixture, $\varepsilon=Q Y+C_{v} T$ the internal energy, $h=Q Y+C_{p} T$ the enthalpy, $Q$ the energy release in the reaction, and $C_{v}, C_{P}$ the specific heats (assumed constant) at constant volume and pressure, respectively. A single irreversible first-order Arrhenius reaction is considered, with the activation energy $E_{a}$ and the constant of time dimension $\tau_{R}$. The stress tensor $\gamma_{i, j}$ and the energy diffusion vector $q_{i}$ are given by

$$
\gamma_{i, j}=\zeta\left(\frac{\partial u_{i}}{\partial x}+\frac{\partial u_{j}}{\partial x_{i}}-\frac{2}{3} \frac{\partial u_{k}}{\partial x_{k}} \delta_{i, j}\right), \quad q_{i}=-\zeta\left(\frac{C_{p}}{\operatorname{Pr}} \frac{\partial T}{\partial x_{i}}+\frac{Q}{\operatorname{Sc}} \frac{\partial Y}{\partial x_{i}}\right)
$$

where $\zeta \equiv \rho v$ is the dynamic viscosity, Pr and Sc are the Prandtl and Schmidt numbers, respectively. The burned and unburned gases are assumed as an ideal gas and have the identical molecular weights. Hence the equation of state is 


$$
P=\rho \frac{R_{u}}{M} T
$$

where $\mathrm{M}$ is molar weight. The heat release in the reaction can be calculated as $Q=C_{p} T_{f}(\Theta-1)$ for given thermal expansion coefficient. The current analysis uses the moderate activation energy $\left(E / R T_{b}=4\right)$ to spread the activation zone over several computational cells [95].

The unstretched laminar burning velocity $S_{L}$ depends on three major chemical parameters of the fuel: $Q, E_{a}$ and $\tau_{R}$. Predefining $Q$ and $E_{a}, \tau_{R}$ can be found such that $M a \ll 1$. In fact, the laminar burning velocity is the solution of eigenvalue problem determined by the boundary conditions. For the detailed solution procedure, one can see the Ref. [96]. It can be understood that all these quantities $\left(Q, E_{a}\right.$ and $\left.\tau_{R}\right)$ were fixed over the entire computational domain. In this dissertation, the eigenvalue problem is solved in each local point, such that in each local point different $S_{L}$ values are obtained by varying $\tau_{R}$ in the space in radial direction, but keep $Q$ and $E_{a}$ constant. Alternatively, it could be done vice versa.

\subsubsection{The Numerical Scheme}

All the basic equations (4.1) - (4.4) can be expressed also in differential conservative form as

$$
\frac{\partial U}{\partial t}+\frac{\partial F}{\partial x_{1}}+\frac{\partial G}{\partial x_{2}}=Q
$$

where

$$
U=\left(\begin{array}{c}
\rho \\
\rho U_{1} \\
\rho U_{2} \\
\rho \varepsilon+\frac{1}{2} \rho\left(U_{1}^{2}+U_{2}^{2}\right) \\
\rho Y
\end{array}\right), F=\left(\begin{array}{c}
\rho u_{1} \\
\rho u_{1}^{2}+P-\gamma_{11} \\
\rho u_{1} u_{2}-\gamma_{21} \\
u_{1}\left(\rho h+\frac{1}{2} \rho\left(u_{1}^{2}+u_{2}^{2}\right)-\gamma_{11}\right)+q_{1}-u_{2} \gamma_{21} \\
\rho u_{1} Y-\frac{\mu}{S c} \frac{\partial Y}{\partial x_{1}}
\end{array}\right)
$$

and 


$$
G=\left(\begin{array}{c}
\rho u_{2} \\
\rho u_{1} u_{2}-\gamma_{12} \\
\rho u_{2}^{2}+P-\gamma_{22} \\
u_{2}\left(\rho h+\frac{1}{2} \rho\left(u_{1}^{2}+u_{2}^{2}\right)-\gamma_{22}\right)+q_{2}-u_{1} \gamma_{12} \\
\rho u_{2} Y-\frac{\mu}{S c} \frac{\partial Y}{\partial x_{2}}
\end{array}\right), Q=\left(\begin{array}{c}
\rho Y \\
0 \\
0 \\
0 \\
0 \\
\tau_{R} \\
\end{array}\right)
$$

For all simulations, the flame was initiated in the form of an initially planar front propagating in a semi-open channel from the closed end to the open one. The Zeldovich-Frank-Kamenetsky (ZFK) analytical solution [26] is used to obtain planar flame front as our initial condition. The walls are adiabatic and nonslip

$$
\text { ก. } \nabla T=0, \quad \mathrm{u}=0,
$$

where $\hat{\mathrm{n}}$ is a normal vector perpendicular to the side walls. Additionally, to avoid weak shock and sound waves, the nonreflecting boundary conditions are selected at the open end of the channel since such an effect could have an influence on the burning and flame acceleration mechanism $[17,95]$.

As shown in Fig. 4.2, the Eulerian mesh is non-uniform, rectangular and structured, and a self-adaptive grid. The computational domain consists of four parts: two gradually (5\%) increasing non-uniform (closed and open ends) and two uniform grids. The flame and flamegenerated pressure wave are situated at the center with two fine-grid regions. The flame moves with the fine grid zone. In order to have a proper resolution and to avoid expensive computational cost, $0.25 L_{f}$ and $0.5 L_{f}$ grid sizes in the axial direction are used for the flame and leading pressure wave, respectively. 


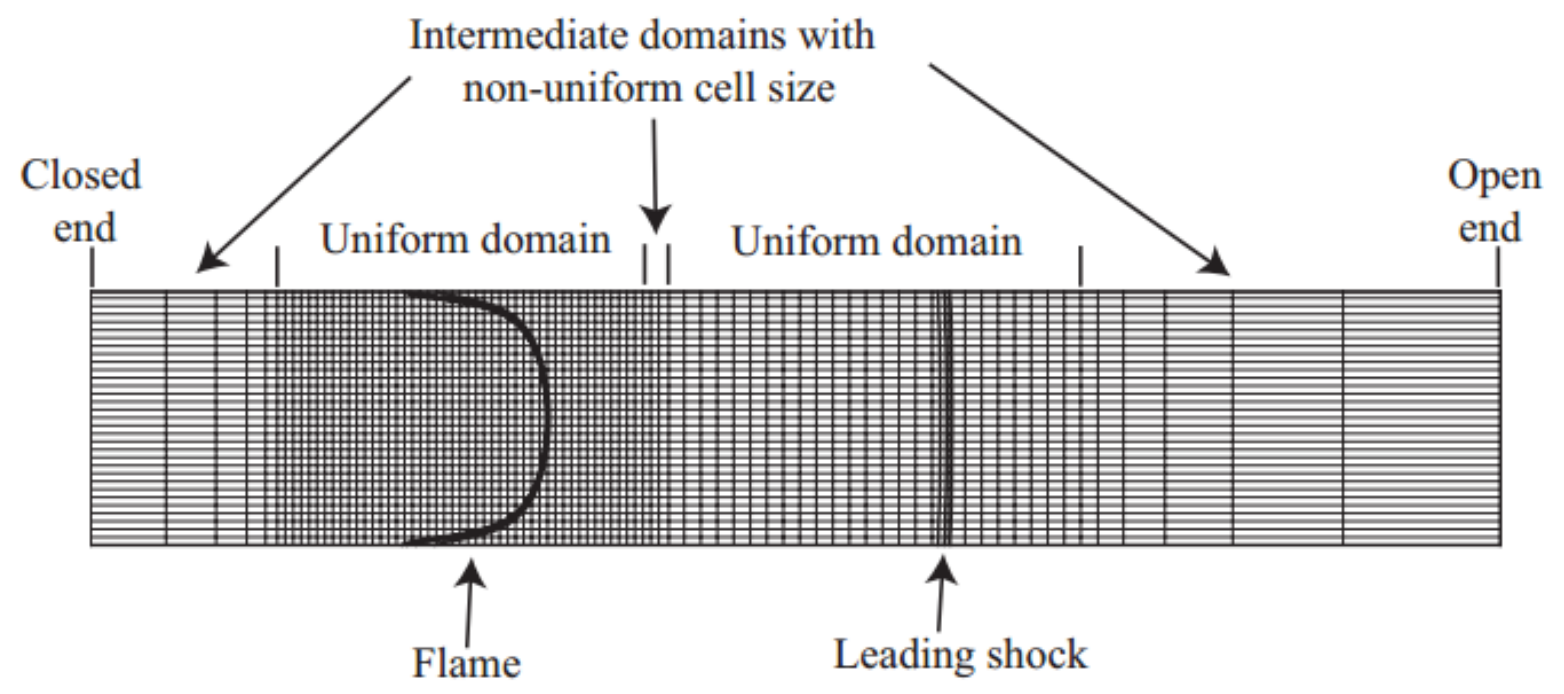

Figure 4.2: Adaptive grid structure [95].

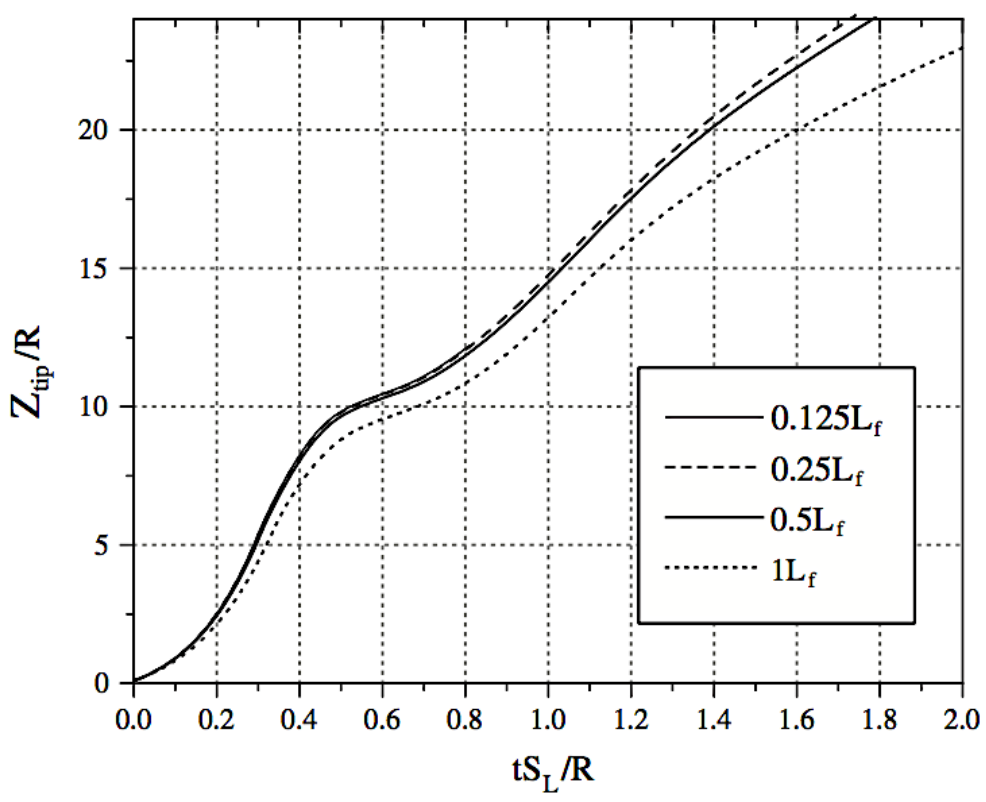

Figure 4.3: Effect of four different flame grid size on the scaled flame tip position [57].

Figure 4.3 exhibits the effect of four different flame grid sizes $\left(0.125 L_{f} ; 0.25 L_{f} ; 0.5 L_{f} ; 1 L_{f}\right)$ on the scaled flame tip position. Results yield a sufficiently refined grid such that resolution is not worse than $5 \%$, and this can be taken as the numerical accuracy of the simulation data 
according to the resolution tests performed in the previous works [57, 97]. In all the following simulation runs, $0.25 L_{f}$ flame grid size is used.

\subsubsection{Concentration Distribution Functions}

The four distributions considered are presented in Fig. 4.4. First, the uniform coal dust concentration distribution, Fig. 4.4(a), is employed, which provides a base model for the computational platform. Subsequently, three non-uniform distributions along the channel as functions of the radial coordinate are considered. In the case of linear coal dust concentration distribution, Fig. 4.4(b) has the maximum dust concentration, $c_{s}=c_{s, \max }$, at the bottom of the channel and no dust at the top of the channel.

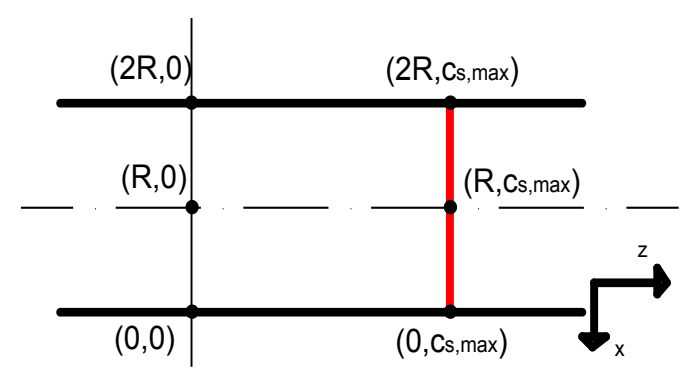

(a)

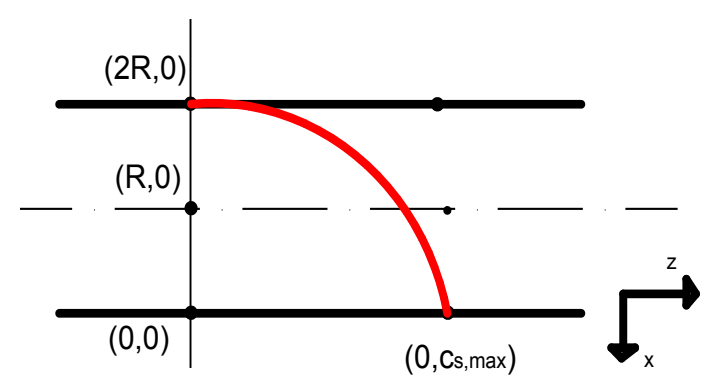

(c)

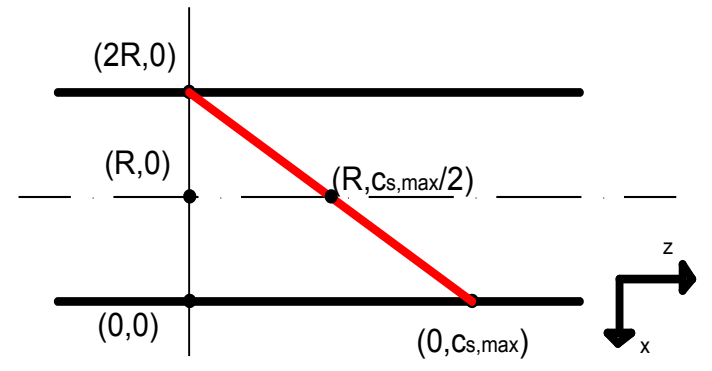

(b)

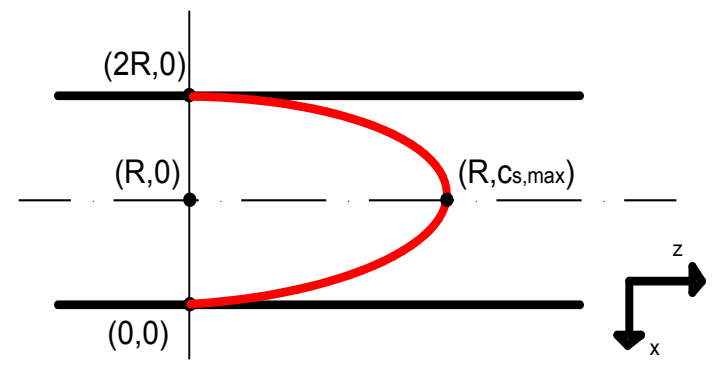

(d)

Figure 4.4: Schematic of the different coal dust concentration distributions:

(a) homogenous; (b) linear; (c) cubic and (d) parabolic [31].

By applying the boundary conditions on the linear gradient of non-uniform dust distribution, one ends up with the following function:

$$
c_{s}=c_{s, \max }\left(1-\frac{x}{2 R}\right) \text {. }
$$


A cubic coal dust concentration distribution, Fig. 4.4(c), is also considered

$$
c_{s}=c_{s, \max }\left[1-\left(\frac{x}{2 R}\right)^{3}\right],
$$

and a parabolic one, Fig. 4.4(d),

$$
c_{s}=c_{s, \max }\left[1-4\left(\frac{x-R}{2 R}\right)^{2}\right] \text {, }
$$

where the maximal dust concentration is attained along the centerline, and it is zero (no dust) along the bottom and top of the channel. With $x=0$, all Eqs. (4.11) - (4.13) obviously yield $c_{s}=c_{s, \max }$ as in Fig. 4.4(a). As pointed out in Sec. 3.1, these dust concentrations distributions do not have the same averaged concentration across the cross section. For example, the linear concentration variation is half of the homogenius one, the cubic concentration variation has a larger averaged concentration than linear variation, and parabolic concentration variation is between linear and cubic variations.

\subsection{Results and Discussion}

The present simulations consider lean $(\phi=0.7)$ methane/air/coal dust combustion, which is relevant to the practical reality $[27,31]$. Additionally, a small particle radius, $r_{s}=10 \mu \mathrm{m}$ with a concentration of $120 \mathrm{~g} / \mathrm{m}^{3}$, providing an effective equivalence ratio promotion due to fast pyrolysis ability, is used. The laminar flame speed for the given equivalence ratio, $\phi=0.7$, in the absence of dust particles was taken as $S_{L}=0.169 \mathrm{~m} / \mathrm{s}$ [62]. This value provides realistically slow flame propagation as compared to the speed of sound (the flame Mach number is $\left.M a=S_{L} / c_{o}=4.87 \times 10^{-4}\right)$. In fact, this flame velocity can be calculated using Eq. (2.44), see Sec. 2, with $c_{s}=0$ or $r_{s}=0$. The thermal expansion in the burning process is taken as $\Theta=6.11$, which is related to methane/coal particle burning for the given equivalence ratio, $\phi=0.7$ as shown in Table 2.2. The standard ("room") initial pressure and temperature are taken as $P_{0}=10^{5} \mathrm{~Pa}$ and $T_{0}=300 \mathrm{~K}$, respectively. The dynamic viscosity and the Prantdl number were

taken as $\zeta=1.7 \times 10^{-5} \mathrm{Ns} / \mathrm{m}^{2}$ and $\operatorname{Pr}=1.0$, respectively, with the Lewis number being 
$L e=\mathrm{Pr} / \mathrm{Sc}=1$. The gas phase is considered to be an ideal gas, $P=\rho R_{u} T / M$, with a constant molecular weight $M=2.9 \times 10^{-2} \mathrm{~kg} / \mathrm{mol}$. The activation energy was chosen as $E_{a}=56 R_{u} T_{f}$.

The flame dynamics are conventionally characterized by the flame Reynolds number

$$
\operatorname{Re}=\frac{R S_{L}^{\text {mean }}}{v}=\frac{R}{\operatorname{Pr} L_{f}},
$$

where $L_{f}=8.65 \times 10^{-5} \mathrm{~m}$ and $R$ is the channel half-width. It was shown that the effect of nonuniform dust distribution becomes substantial when the channel width is no less than $24 L_{f}$, so this value of $\operatorname{Re}=24$ is used in the present simulations [31].

Figures 4.5 and 4.6 compare the characteristics of the accelerating flames for different dust distributions. Specifically, Fig. 4.5 shows the velocity of the flame position at the centerline, $S_{L}$, scaled by $S_{L}$, versus the scaled time, $\tau=t S_{L, \text { mean }} / R$. First of all, it observed that the parabolic dust distribution moderates flame acceleration when compared to a homogeneous case. However, the most important observation is a sudden jump of the flame velocity, around $\tau \sim 0.25-0.4$, for the homogeneous, linear and cubic distributions. Indeed, the flame velocity grows almost by an order of magnitude for methane/air/dust combustion. At the center, flame velocity increases, but later on it decreases and again increases. The answers for such behavior will be addressed later in this section through the analysis of the flame evaluation snapshots. Also, the plots for the linear and cubic distributions closely resemble each other, except for a minor quantitative deviation. The case of no dust is also presented, which showed similarity with the homogeneous distribution.

However, to have a more informative knowledge on the flame propagation and acceleration of all distributions, the evolution of the scaled flame front surface area, $A_{w} / 2 R$ is investigated as presented in Fig. 4.6. The growth of the flame surface area promotes its velocity. In the case of a parabolic distribution, the lower flame surface area mitigates flame acceleration as depicted in Fig. 4.5. Initially, growth of the flame surface increases in a similar fashion for homogeneous, linear and cubic. Later, around $\tau \sim 0.4$, the growth of the flame surface becomes much stronger for linear and cubic distributions as compared to the homogenous one. As a result, the higher the flame surface area, the faster the flame propagates and accelerates, or vice versa. 


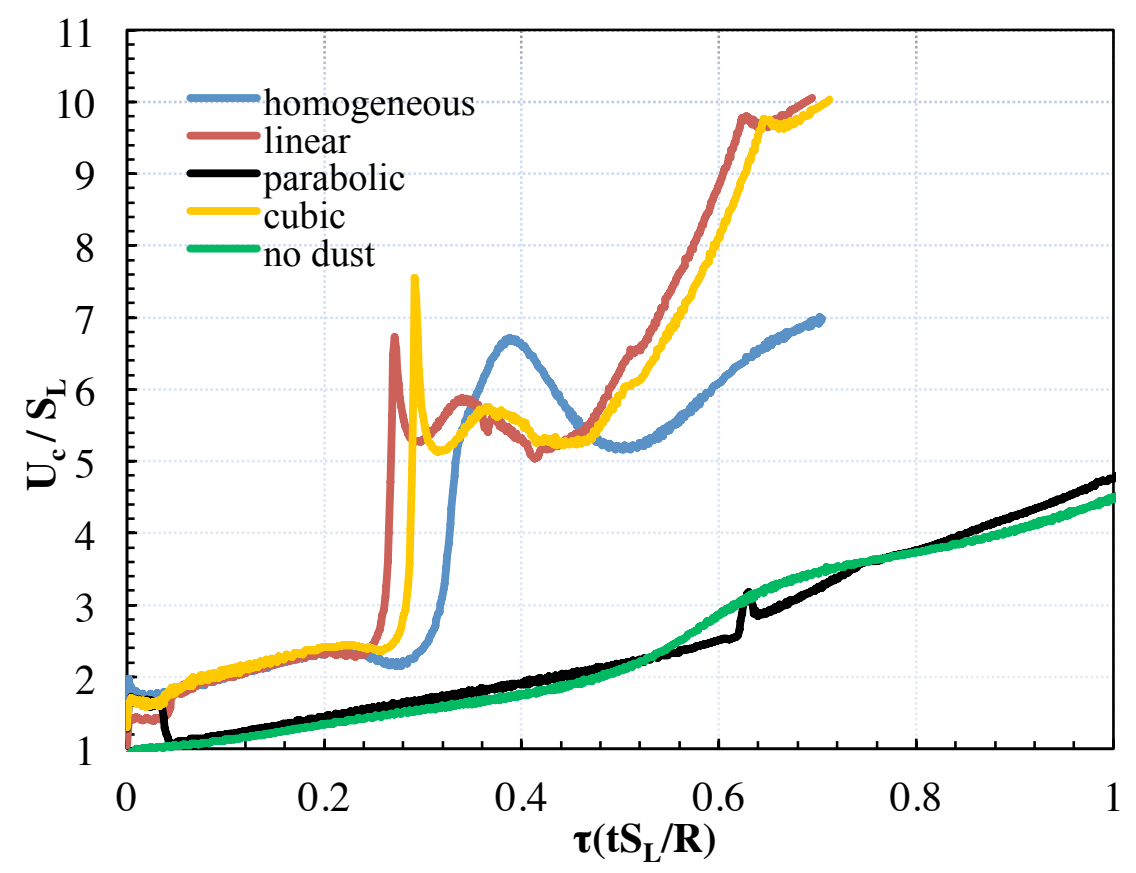

Figure 4.5: The scaled flame velocity at the centerline $U_{c} / S_{L}$ versus the scaled time $t S_{L} / R$ for various coal dust concentration distributions.

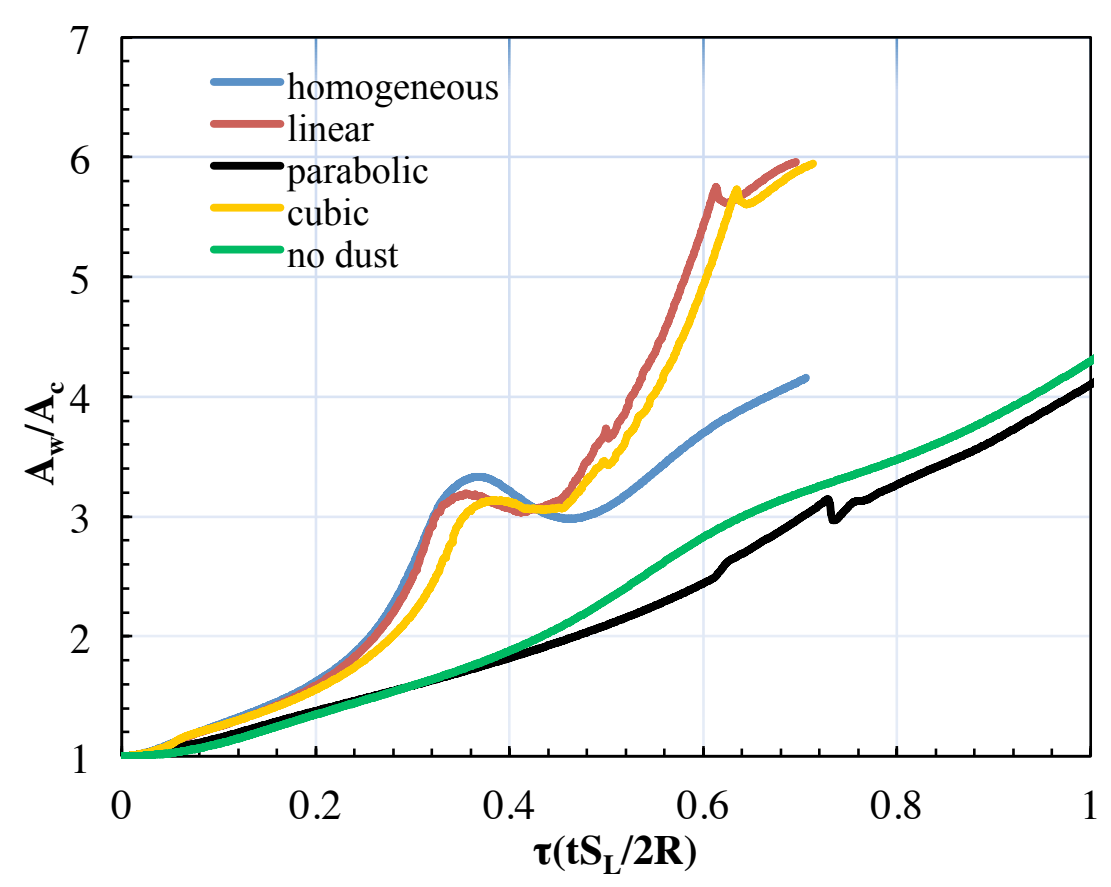

Figure 4.6: The scaled flame surface area $A_{\mathrm{w}} / 2 R$ versus the scaled time $t S_{L} / R$ for various coal dust concentration distributions.

Which mechanism is responsible for such a trend? To answer this question, the evolution of the flame shape is investigated for all distributions at identical time instants, as shown in Fig. 4.7. 
The no dust case is also presented. Here, the colors characterize the temperature variation. For all simulation runs, the flame was initiated in the form of an initially planar front propagating in a semi-open channel, from its closed end to the open one; see Fig. 4.7(a). Subsequently, the flame front becomes corrugated due to wall friction, thus generating a non-uniform flow velocity field.

For the homogeneous distribution, the formation of a trough is observed, which gets deeper with time. Later, the central segment of the flame accelerates faster than the upper and lower ones; see Fig. 4.7(d). At the end, only a shallow trough is visible. The origin of the trough may be attributed to the $D L$ instability allowable by $\operatorname{Re}_{f}=24[17,31]$. A similar trough formation is observed for the case of no dust particles at the later stages, Fig. 4.7(d-e), however trough formation is significantly weaker as compared to the homogeneous distribution. It is noted that a non-uniform distribution of dust particles makes the shape of the flame front much more intriguing. Specifically, the linear and cubic distributions lead to the formation of an asymmetric flame front, due to a higher concentration of the combustible particles in a lower half of the channel. Acceleration is strong in the lower branch in all directions so that it catches the upper part later on. The snapshots of the flame evolution show that the trough formation, and a loss of symmetry of the flame front, originates in the region close to the flame cusp. At the end of the simulations, a smooth but still non-symmetrical flame front shape is observed for the linear and cubic concentration distributions. While the linear and cubic cases resemble each other, the linear distribution triggers the flame acceleration slightly more than the cubic distribution.

Regarding the effect of the parabolic dust concentration distribution, the dense combustible particles are distributed through the center of the channel while their concentration decreases towards the upper and lower sidewalls. After the ignition of the fuel mixture, an intrinsically unstable flame front attempts to generate a trough. However, the dust particles located at the centerline promote the flame velocity locally, and thereby prevent through formation. In other words, the parabolic dust concentration distribution stabilizes an intrinsically instable flame front similar to Huygens flame stabilization principle. Consequently, the increase in the flame surface area appears slower, thereby moderating flame acceleration as compared to other distributions considered. 


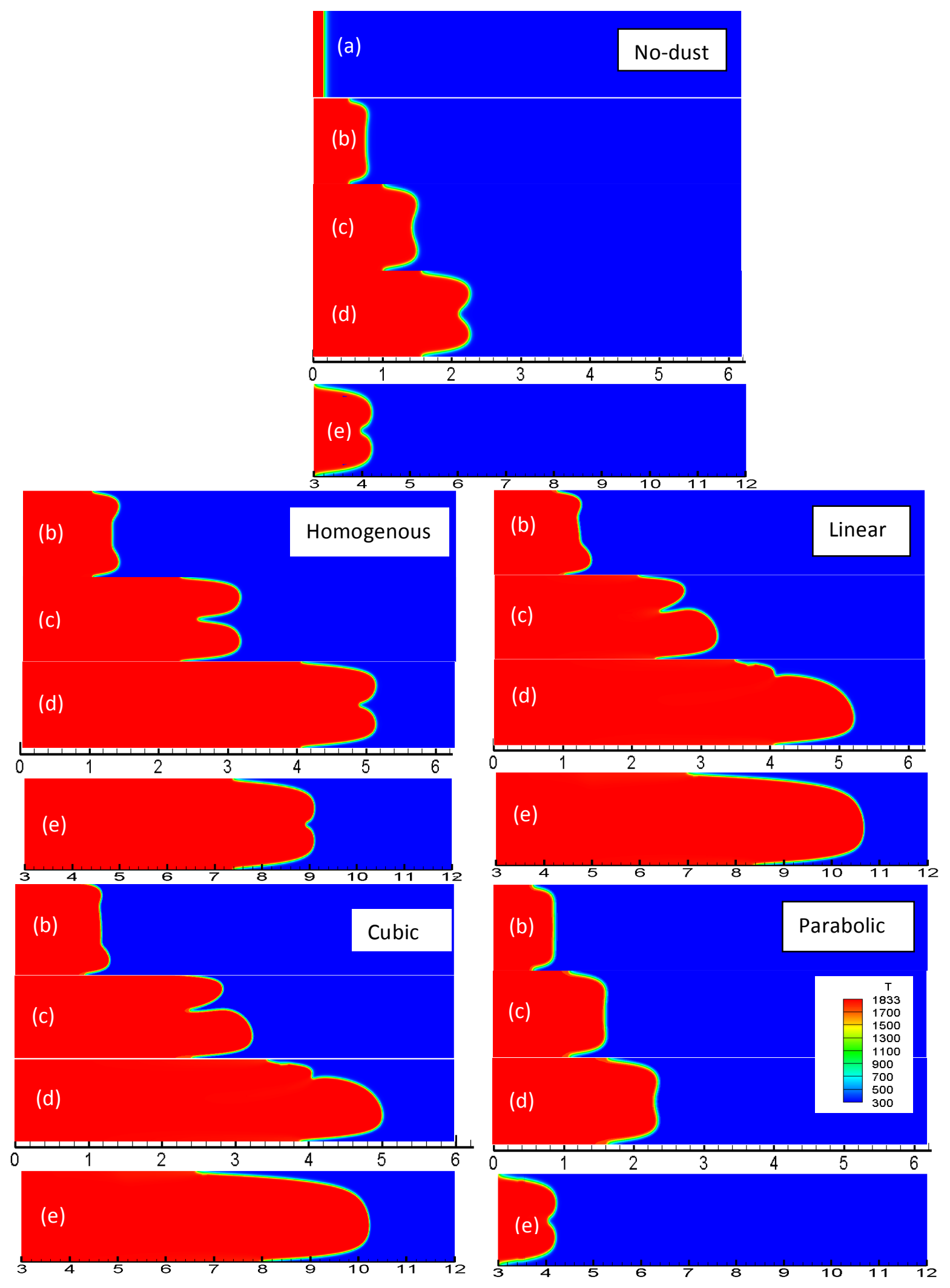

Figure 4.7: Temperature snapshots at time: $\tau=0$ (a), 0.15 (b), 0.27 (c), 0.36 (d) and 0.61 (e). 


\section{Conclusion}

This dissertation focused on the interplay between combustion instabilities, and combustible and/or inert coal dust impurities and their influence on the processes of flame acceleration and detonation triggering. It also analyzes how local variations of the flame properties can control the flame morphology and dynamics. Through analysis of a deflagration regime, the aim is to develop a predictive scenario for a methane/air/dust fire, and to refine the safety standards in the coalmining industry. The latter is exceptionally important for West Virginia (and all mining related states and countries), where a large portion of the state economy is based on coal mines. This thesis has undertaken a step towards developing a predictive quantitative scenario of burning accidents in coal mines.

For this purpose, the analysis of globally-spherical expanding corrugated flames was combined with the finger flame formulation (Section 2). The study considered both the 2Dplanar (Sec. 2.1.1), and the cylindrical-axisymmetric (Sec. 2.1.2) geometries. A higher flame tip velocity and, thereby stronger acceleration, were attained in the cylindrical geometry when compared to a 2D-planar one. While it is methane that typically explodes in coal mines, propane burning is also considered herein, for comparison. Starting with purely gaseous combustion (Sec. 2.1 and 2.2), analysis later extended to the coal-dust/gas environments (Sec. 2.3) by means of the Seshadri formulation [30] for the unstretched laminar flame velocity. In this respect, combustible (i.e., coal) and inert (i.e., sand) dust particles of various concentrations $\left(0-300 \mathrm{~g} / \mathrm{m}^{3}\right)$ and sizes $(10-75 \mu \mathrm{m})$, as well as their combination, are investigated. It is noted that the small particles, of radius $r_{s}=10 \mu \mathrm{m}$, combustible or inert, influence flame spreading noticeably more strongly than the larger particles, of radius $r_{s}=75 \mu \mathrm{m}$. The instantaneous flame tip velocity grows with the concentration of the combustible dust as long as the concentration does not exceed $250 \mathrm{~g} / \mathrm{m}^{3}$. In contrast, the flame velocity monotonically decreases with the concentration of the inert dust. In particular, for a given particle size $r_{s}=25 \mu \mathrm{m}$ and equivalence ratio $\phi=0.7$, the increase or decrease in the concentration does not impact the flame velocity due to a balance between the effects of the equivalence ratio and the heat sink. 
The evolution and velocity of the tip and skirt of the flame front as well as the locus and timing of a potential detonation onset have been predicted and quantified as they are key stages and characteristics of coal mine burning. The timing for each stage, as well as the flame shapes, propagation speeds, acceleration rates, run-up distances and flame-generated velocity profiles were identified. Specifically, in the event of an accidental ignition in a coal mine, first, an embryonic flame develops from a smooth hemispherical/hemi-circular kernel to a globallyspherical/cellular (corrugated) structure. This occurs due the Darrieus-Landau flame instability, and the process is accompanied by self-similar flame acceleration. Subsequently, such a cellular flame acquires a finger-like shape, exhibiting strong acceleration, of short duration, until a flame skirt contacts a passage wall. In particular, in a 2D geometry, no detonation is predicted for methane combustion, while in the case of propane, the detonation may occur for nearstoichiometric, slightly fuel-rich burning, with equivalence ratios in the range $1 \leq \phi \leq 1.2$. For the cylindrical geometry, this range varies as $0.9 \leq \phi \leq 1.2$ for methane and $0.8 \leq \phi \leq 1.3$ for propane. The entire acceleration scenario may promote the total burning rate by up to two orders of magnitude, to near-sonic flame velocities. Such fast flame spreading can constitute to a tremendous disaster, especially in enclosed areas with limited space, such as coal mines. Moreover, in addition to the direct disaster of such a fast flame, it may facilitate the deflagrationto-detonation transition (DDT), thereby leading to additional hazards such as the spread of strong shock waves putting personnel and equipment at risk. In most cases, shock waves are the cause of the majority of fatalities in coal mines.

In Sec. 2.4, the incompressible theory of methane/air/dust flame acceleration in a coal mining passage is revisited by incorporating the effect of gas compression into the analysis, and the original incompressible formulation is thereby validated. It is shown that gas compression moderates flame acceleration, and its impact depends on the type of the fuel, geometry and various thermal-chemical parameters. The relative role of gas compression is quantified for a variety of parameters such as $\phi, r_{s}, c_{d}$, and it appeared to be stronger for dusty-gaseous environments when compared to purely gaseous combustion.

As a result, in Sec. 2.5, the intrinsic accuracy of the formulation is identified for a given set of parameters. Specifically, moderation of flame acceleration, due to gas compression, is more effective in stoichiometric mixtures, such that the decrease in the maximal flame tip position 
$Z_{\text {tip }}\left(t_{\text {wall }}\right)$ varies in the range $43 \sim 69 \%$ for methane- and propane/air flames. This moderation is relatively minor for the lean $(\phi=0.63)$ and rich $(\phi=1.4)$ mixtures, being $5-22 \%$ and $15-47 \%$, respectively. It is also noted that while flames accelerate faster in a cylindrical geometry, moderation of acceleration due to compressibility is also larger in this geometry than in a 2D case. Furthermore, in contrast to the incompressible predictions, such moderation reduces the flame tip velocity $U_{\text {tip }}\left(t_{\text {wall }}\right)$ far beyond the sound speed irrespective of the type of a fuel, equivalence ratio, and geometry.

The analysis is then extended from gaseous to gaseous-dusty combustion. Specifically, both combustible and inert dust particles, as well as their combination are considered, and it is shown how the particle size and concentration influence the flame dynamics and compressibility effect. Namely, the role of compressibility is stronger for small particles, $r_{s}=10 \mu \mathrm{m}$, when compared to that with $r_{s}=75 \mu \mathrm{m}$. Although when compared to the particle size, the particle concentration is less sensitive to the effect of gas compression, it is noted that the role of compressibility strengthens with the increasing concentration of the combustible particles. In contrast, the effect of compressibility decreases with an increase in the concentration of the inert particles.

Both incompressible and compressible analytical formulations in Sec. 2 are steps towards the comprehensive coalmining fire evolution scenario, with some factors left beyond the study. Namely, the present analysis is conceptually laminar, while a real fire flame in a coal mine is presumably turbulent. The role of turbulence may potentially be incorporated into the present formulation by replacing the planar flame speed $S_{L}$ by the local turbulent burning velocity $S_{T}$, with the ratio $S_{T} / S_{L}$ obtained analytically, phenomenologically, from an experiment, or computationally from a relevant turbulent flame speed model. On the other hand, the fire evolution maybe influenced, positively or negatively, by the different motions of dust particles. In particular, this may lead to a non-uniform distribution of the dust concentration and thereby spatial and/or temporal variations of the local thermal-chemical parameters such as the equivalence ratio and the laminar burning velocity. In fact, this is indeed the case in coal mines where the coal dust distribution is typically non-uniform, and a steady dense coal dust layer may spread through the bottom of the passage. 
In Sec. 3, spatial variations of laminar flame speed $S_{L}$ are studied to account for the potential impacts of pressure/temperature variations as well as the non-uniform distribution of the equivalence ratio and/or of combustible or inert dust impurities as a general fundamental work. To do this, various spatial distributions (constant, linear, parabolic and hyperbolic) of the planar flame speed $S_{L}$ are incorporated into the theory of flame acceleration due to a finger-like flame shape. Revisited formulae for the flame skirt and the flame tip are obtained for both the 2D-planar, Sec. 3.1.1, and cylindrical-axisymmetric, Sec. 3.1.2, cases. These results are presented and compared in Sec. 3.2. It is demonstrated, qualitatively and quantitatively, that the geometry influences the flame acceleration mechanisms. Specifically, in the axisymmetric geometry a flame accelerates faster when compared to the $2 \mathrm{D}$ one for all flame speed distributions considered, including the $S_{L}=S_{L}^{0}=$ const case. In summary, the concept of spatial variation of the unstretched laminar flame speed $S_{L}$ can moderate or promote the global flame acceleration mechanism. Indeed, different imposed $S_{L}$ - functions may provide different controlling strategies to mitigate flame acceleration. As a result, imposing various $S_{L}$ distributions can potentially appear a useful tool to control flame acceleration. In this dissertation, this new research direction is initiated by imposing the linear, parabolic and hyperbolic spatial $S_{L}$ - distributions and comparing them to each other and to the uniform one. As stated earlier, practical applications include, in particular, the methane/air fire safety issues in coal mines with dusty atmosphere.

Section 4 is devoted to flame propagation in a combined gaseous-dusty environment with non-uniform coal dust concentration distributions by means of the computational simulations for a particular set of parameters. While a parametric study can be conducted easily by means of analytical studies, a computational study can provide a more detailed analysis of physical mechanisms that may not be described by analytical studies. The numerical approach employs a finite-volume, Navier-Stokes code, updated for parallel computations and solving for the reacting flow equations with fully-compressible hydrodynamics, transport properties, and Arrhenius chemical kinetics as described in Sec. 4.2. Specifically, flame acceleration due to wall friction is analyzed for the linear, parabolic and cubic coal dust concentration distributions; the similarity and differences in the evolutions of the flame morphology and velocity in each of 
these cases as well as in the case of purely gaseous combustion are identified in Sec. 4.3. It is shown that a non-uniform dust distribution may result in an extra distortion or local stabilization of the flame front, which respectively increases or decreases the total surface area of the flame front, thereby promoting or moderating the flame acceleration scenario. At the end of the analyses, it can be concluded that the effect of different concentration distributions (homogenous, linear, cubic and parabolic) has a stronger effect on the morphology and dynamics of flame evolution when compared to the effect of concentration distributions with the same average concentration across the cross section. In a future study, one can investigate the later stages of flame acceleration due to wall friction, which may provide other significant insights. Moreover, the effect of the particle radius, concentration and different distribution functions can also be examined in a set of parametric simulations. It would also be interesting to investigate the effect of inert particles and their distributions. While the reader may expect some validation of the provided computational platform, unfortunately, to the best of my knowledge no suitable experimental studies exist at the moment, and it is hoped that the present dissertation will provide a good platform for more elaborated experiments for combustion of non-uniformly distributed coal particles in a gaseous mixture.

Consequently, it is believed that the present dissertation will provide a considerable physical insight not only for the understanding of accidental coal mine flames but also for applications associated with the controlling combustion strategies. 


\section{References}

[1] J. Warnatz, U. Maas, and R. W. Dibble, Combustion: Physical and chemical fundamentals, modeling and simulation, experiments, pollutant formation, 2nd Edition. Berlin: Springer, 1999.

[2] C. K. Law, Combustion physics, 1st Edition. New York: Cambridge, 2006.

[3] M. Liberman, Introduction to physics and chemistry of combustion- explosion, flame, detonation, 1st Edition. Berlin: Springer, 2008.

[4] O. J. Ugarte-Almeyda, "Effect of boundary conditions on propagation and morphology of premixed flames in narrow conduits," Doctoral Thesis, West Virginia University, Morgantown, USA, 2015.

[5] V. Bychkov and M. Liberman, "Dynamics and stability of premixed flames," Phys. Rep., vol. 325, no. 4-5, pp. 115-237, 2000.

[6] T. Poinsot and D. Veynante, Theoretical and numerical combustion, 2nd Edition. Philadelphia: Edwards, 2005.

[7] V. Akkerman, V. Bychkov, A. Petchenko, and L. E. Eriksson, "Flame oscillations in tubes with nonslip at the walls," Combust. Flame, vol. 145, no. 4, pp. 675-687, 2006.

[8] V. Bychkov, "Importance of the Darrieus-Landau instability for strongly corrugated turbulent flames," Phys. Rev. E, vol. 68, no. 6, p. 66304, 2003.

[9] A. Khokhlov, E. Oran, and G. Thomas, "Numerical simulation of deflagration-todetonation transition: the role of shock-flame interactions in turbulent flames," Combust. Flame, vol. 117, no. 1-2, pp. 323-339, 1999.

[10] J. P. McGarry and K. A. Ahmed, "Flame-turbulence interaction of laminar premixed deflagrated flames," Combust. Flame, vol. 176, pp. 439-450, 2017.

[11] A. Petchenko, V. Bychkov, V. Akkerman, and L. E. Eriksson, "Violent folding of a flame front in a flame-acoustic resonance," Phys. Rev. Lett., vol. 97, no. 16, p. 164501, 2006.

[12] A. Petchenko, V. Bychkov, V. Akkerman, and L. E. Eriksson, "Flame-sound interaction in tubes with nonslip walls," Combust. Flame, vol. 149, no. 4, pp. 418-434, 2007.

[13] C. F. Silva, T. Emmert, S. Jaensch, and W. Polifke, "Numerical study on intrinsic thermoacoustic instability of a laminar premixed flame," Combust. Flame, vol. 162, no. 9, pp. 3370-3378, 2015.

[14] G. Ciccarelli, C. T. Johansen, and M. Parravani, "The role of shock-flame interactions on flame acceleration in an obstacle laden channel," Combust. Flame, vol. 157, no. 11, pp. 2125-2136, 2010. 
[15] H. Wei, D. Gao, L. Zhou, D. Feng, and R. Chen, "Different combustion modes caused by flame-shock interactions in a confined chamber with a perforated plate," Combust. Flame, vol. 178, pp. 277-285, 2017.

[16] V. Bychkov and M. Liberman, "Stability and the fractal structure of a spherical flame in a self-similar regime," Phys. Rev. Lett., vol. 76, no. 15, pp. 2814-2817, 1996.

[17] V. Bychkov, A. Petchenko, V. Akkerman, and L. E. Eriksson, "Theory and modeling of accelerating flames in tubes," Phys. Rev. E, vol. 72, no. 4, p. 46307, 2005.

[18] V. Akkerman, V. Bychkov, A. Petchenko, and L. E. Eriksson, "Accelerating flames in cylindrical tubes with nonslip at the walls," Combust. Flame, vol. 145, no. 1-2, pp. 206219, 2006.

[19] D. Valiev, V. Bychkov, V. Akkerman, C. K. Law, and L. E. Eriksson, "Flame acceleration in channels with obstacles in the deflagration-to-detonation transition," Combust. Flame, vol. 157, no. 5, pp. 1012-1021, 2010.

[20] V. N. Gamezo, T. Ogawa, and E. S. Oran, "Numerical simulations of flame propagation and DDT in obstructed channels filled with hydrogen-air mixture," Proc. Combust. Inst., vol. 31 II, pp. 2463-2471, 2007.

[21] E. S. Oran and V. N. Gamezo, "Origins of the deflagration-to-detonation transition in gasphase combustion," Combust. Flame, vol. 148, no. 1-2, pp. 4-47, 2007.

[22] G. Darrieus, "Propagation d'un front de flamme," La Technique Moderne and Congrés de Mécanique Appliquée Paris, 1938.

[23] L. Landau, "On the theory of slow combustion," Acta Physicochim. URSS, vol. 19, no. 1, pp. 77-85, 1944.

[24] P. Pelce and P. Clavin, "Influence of hydrodynamics and diffusion upon the stability limits of laminar premixed flames," J. Fluid Mech., vol. 124, pp. 219-237, 1982.

[25] Y. Zeldovich, G. Barenblatt, V. Librovich, and G. Makhviladze, The mathematical theory of combustion and explosions, 1st Edition. New York, 1985.

[26] Y. Zeldovich and D. Frank-Kamenetski, "A theory of thermal propagation of flames," Acta Physicochim. URSS, vol. 12, pp. 341-350, 1938.

[27] S. Demir, V. Bychkov, S. H. R. Chalagalla, and V. Akkerman, "Towards a predictive scenario of a burning accident in a mining passage," Combust. Theory Model., pp. 1-26, 2017- Published Online.

[28] S. Demir, A. Calavay, and V. Akkerman, "Influence of gas compressibility on a burning accident in a mining passage," Combust. Theory Model., 2017-Accepted.

[29] S. Demir, H. Sezer, and V. Akkerman, "Effect of local variations of the laminar flame 
speed on the global finger-flame acceleration scenario," Combust. Theory Model., 2017Under Review

[30] K. Seshadri, A. Berlad, and V. Tangirala, "The structure of premixed particle-cloud flames," Combust. Flame, vol. 89, no. 3-4, pp. 333-342, 1992.

[31] S. Demir, H. Sezer, T. Bush, and V. Akkerman, "Promotion and mitigation of premixed flame acceleration in dusty-gaseous environment with various combustible dust distributions: A computational study," in 26th ICDERS, Boston, USA, 2017, pp. 1-6.

[32] V. Bychkov, V. Akkerman, G. Fru, A. Petchenko, and L. E. Eriksson, "Flame acceleration in the early stages of burning in tubes," Combust. Flame, vol. 150, no. 4, pp. 263-276, 2007.

[33] V. Akkerman, C. K. Law, and V. Bychkov, "Self-similar accelerative propagation of expanding wrinkled flames and explosion triggering," Phys. Rev. E, vol. 83, no. 2, p. 26305, 2011.

[34] S. H. R. Chalagalla, "Predictive scenario for premixed methane-air flame spreading and explosion triggering in a mining passage," Masters Thesis, West Virginia University, 2015.

[35] A. Calavay, "Effect of gas compression on mining fire scenario in gaseous and dustygaseous environments," Masters Thesis, West Virginia University, 2015.

[36] M. Faraday and C. Lyell, "Explosions in coal mines," Philos. Mag., vol. 26, no. 4, pp. 16$35,1845$.

[37] K. Chatrathi, J. E. Going, B. Grandestaff, F. Corporation, S. Street, and B. Springs, "Flame Propagation in industrial scale piping," Process Saf. Prog., vol. 20, no. 4, pp. 286294, 2001.

[38] M. Silvestrini, B. Genova, G. Parisi, and F. Leon Trujillo, "Flame acceleration and DDT run-up distance for smooth and obstacles filled tubes," J. Loss Prev. Process Ind., vol. 21, no. 5, pp. 555-562, 2008.

[39] X. Chen, Y. Zhang, and Y. Zhang, "Effect of CH4-air ratios on gas explosion flame microstructure and propagation behaviors," Energies, vol. 5, no. 10, pp. 4132-4146, 2012.

[40] M. Bi, C. Dong, and Y. Zhou, "Numerical simulation of premixed methane-air deflagration in large L/D closed pipes," Appl. Therm. Eng., vol. 40, pp. 337-342, 2012.

[41] L. Kjäldman, "Numerical flow simulation of dust deflagrations," Powder Technol., vol. 71, no. 2, pp. 163-169, 1992.

[42] Q. Liu, C. Bai, X. Li, L. Jiang, and W. Dai, "Coal dust/air explosions in a large-scale tube," Fuel, vol. 89, no. 2, pp. 329-335, 2010. 
[43] T. Skjold, D. Castellanos, K. L. Olsen, and R. K. Eckhoff, "Experimental and numerical investigation of constant volume dust and gas explosions in a $3.6 \mathrm{~m}$ flame acceleration tube," J. Loss Prev. Process Ind., vol. 30, no. 1, pp. 164-176, 2014.

[44] R. W. Houim and E. S. Oran, "Structure and flame speed of dilute and dense layered coaldust explosions," J. Loss Prev. Process Ind., vol. 36, pp. 214-222, 2015.

[45] B. Gardner, R. Winter, and M. Moore, "Explosion development and deflagration-todetonation transition in coal dust/air suspensions," Symp. Combust., vol. 21, no. 1, pp. 335-343, 1988.

[46] W. Bartknecht, Bundesinstitut fur arbeitsschutz (Bifa). Koblenz, Germany, 1971.

[47] W. Bartknecht, Staubexplosionen. Verlag: Springer, 1987.

[48] M. J. Ajrash, J. Zanganeh, and B. Moghtaderi, "Deflagration of premixed methane-air in a large scale detonation tube," Process Saf. Environ. Prot., vol. 109, pp. 374-386, 2017.

[49] M. J. Ajrash, J. Zanganeh, and B. Moghtaderi, "The flame deflagration of hybrid methane coal dusts in a large-scale detonation tube (LSDT)," Fuel, vol. 194, pp. 491-502, 2017.

[50] M. J. Ajrash, J. Zanganeh, and B. Moghtaderi, "Impact of suspended coal dusts on methane deflagration properties in a large scale straight duct," J. Hazard. Mater., vol. 338, pp. 334-342, 2017.

[51] A. Oppenheim, A. Laderman, and P. Urtiew, "The onset of retonation," Combust. Flame, vol. 6, pp. 193-197, 1962.

[52] P. Urtiew and A. Oppenheim, "Experimental observations of the transition to detonation in an explosive gas," Proc. R. Soc. A Math. Phys. Eng. Sci., vol. 295, no. 1440, pp. 13-28, 1966.

[53] C. Clanet and G. Searby, "On the 'tulip flame' phenomenon," Combust. Flame, vol. 105, no. 1-2, pp. 225-238, 1996.

[54] H. Xiao, R. W. Houim, and E. S. Oran, "Formation and evolution of distorted tulip flames," Combust. Flame, vol. 162, no. 11, pp. 4084-4101, 2015.

[55] D. Bradley, T. Cresswell, and J. Puttock, "Flame acceleration due to flame-induced instabilities in large-scale explosions," Combust. Flame, vol. 124, no. 4, pp. 551-559, 2001.

[56] T. Becker and F. Ebert, "Vergleich zwischen experiment und theorie der explosion grober, freier gaswolken," Chemie Ing. Tech., vol. 57, no. 1, pp. 42-45, 1985.

[57] D. Valiev, V. Akkerman, M. Kuznetsov, L. E. Eriksson, C. K. Law, and V. Bychkov, "Influence of gas compression on flame acceleration in the early stage of burning in tubes," Combust. Flame, vol. 160, no. 1, pp. 97-111, 2013. 
[58] V. Akkerman and V. Bychkov, "Turbulent flame and the darrieus-landau instability in a three-dimensional flow," Combust. Theory Model., vol. 7, no. 4, pp. 767-794, 2003.

[59] M. Liberman, M. Ivanov, A. Kiverin, M. Kuznetsov, A. Chukalovsky, and T. Rakhimova, "Deflagration-to-detonation transition in highly reactive combustible mixtures," Acta Astronaut., vol. 67, no. 7-8, pp. 688-701, 2010.

[60] Z. Chen and Y. Ju, "Theoretical analysis of the evolution from ignition kernel to flame ball and planar flame," Combust. Theory Model., vol. 11, no. 3, pp. 427-453, 2007.

[61] M. P. Burke, Z. Chen, Y. Ju, and F. L. Dryer, "Effect of cylindrical confinement on the determination of laminar flame speeds using outwardly propagating flames," Combust. Flame, vol. 156, no. 4, pp. 771-779, 2009.

[62] S. G. Davis, J. Quinard, and G. Searby, "Markstein numbers in counterflow, methane- and propane- air flames: A computational study," Combust. Flame, vol. 130, no. 1-2, pp. 123136, 2002.

[63] K. L. Cashdollar, "Overview of dust explosibility characteristics," J. Loss Prev. Process Ind., vol. 13, no. 3-5, pp. 183-199, 2000.

[64] K. L. Cashdollar, M. J. Sapko, E. S. Weiss, and M. L. Harris, "Recommendations for a new rock dusting standard to prevent coal dust explosions in intake airways," Pittsburgh, 2010 .

[65] S. Ranganathan, M. Lee, V. Akkerman, and A. S. Rangwala, "Suppression of premixed flames with inert particles," J. Loss Prev. Process Ind., vol. 35, pp. 46-51, 2015.

[66] C. Morley, “Gaseq.”- Web site: http://www.gaseq.co.uk , 2005.

[67] Y. Xie, V. Raghavan, and A. S. Rangwala, "Study of interaction of entrained coal dust particles in lean methane-air premixed flames," Combust. Flame, vol. 159, no. 7, pp. 2449-2456, 2012.

[68] G. Ciccarelli and S. Dorofeev, "Flame acceleration and transition to detonation in ducts," Prog. Energy Combust. Sci., vol. 34, pp. 499-550, 2008.

[69] M. Kuznetsov, M. Liberman, and I. Matsukov, "Experimental study of the preheat zone formation and deflagration to detonation transition," Combust. Sci. Technol., vol. 182, no. 11-12, pp. 1628-1644, 2010.

[70] M. Liberman, G. Sivashinsky, D. Valiev, and L. Eriksson, "Numerical simulation of deflagration-to-detonation transition: the role of hydrodynamic instability," Int. J. Transp. Phenom., vol. 8, pp. 253-277, 2006.

[71] V. Bychkov, V. Akkerman, D. Valiev, and C. K. Law, "Role of compressibility in moderating flame acceleration in tubes," Phys. Rev. E, vol. 81, no. 2, p. 26309, 2010. 
[72] J. D. Anderson, Modern compressible flow: with histrorical perspective, 2nd Edition. New York: McGraw-Hill, 1982.

[73] R. W. Houim and E. S. Oran, "Numerical simulation of dilute and dense layered coal-dust explosions," Proc. Combust. Inst., vol. 35, pp. 2083-2090, 2015.

[74] V. N. Kurdyumov and C. Jiménez, "Propagation of symmetric and non-symmetric premixed flames in narrow channels: Influence of conductive heat-losses," Combust. Flame, vol. 161, no. 4, pp. 927-936, 2014.

[75] C. E. Frouzakis, N. Fogla, A. G. Tomboulides, C. Altantzis, and M. Matalon, "Numerical study of unstable hydrogen/air flames: Shape and propagation speed," Proc. Combust. Inst., vol. 35, no. 1, pp. 1087-1095, 2015.

[76] M. Metghalchi and J. C. Keck, "Burning velocities of mixtures of air with methanol, isooctane, and indolene at high pressure and temperature," Combust. Flame, vol. 48, pp. 191-210, 1982.

[77] R. Sankaran, H. G. Im, E. R. Hawkes, and J. H. Chen, "The effects of non-uniform temperature distribution on the ignition of a lean homogeneous hydrogen-air mixture," Proc. Combust. Inst., vol. 30, no. 1, pp. 875-882, 2005.

[78] S. R. Rockwell and A. S. Rangwala, "Modeling of dust air flames," Fire Saf. J., vol. 59, pp. 22-29, 2013.

[79] J. Grune, K. Sempert, H. Haberstroh, M. Kuznetsov, and T. Jordan, "Experimental investigation of hydrogen-air deflagrations and detonations in semi-confined flat layers," J. Loss Prev. Process Ind., vol. 26, no. 2, pp. 317-323, 2013.

[80] J. Grune, K. Sempert, M. Kuznetsov, and T. Jordan, "Experimental investigation of fast flame propagation in stratified hydrogeneair mixtures in semi-confined flat layers," $J$. Loss Prev. Process Ind., vol. 26, no. 6, pp. 1442-1451, 2013.

[81] X. Shi, J.-Y. Chen, and Z. Chen, "Numerical study of laminar flame speed of fuelstratified hydrogen/air flames," Combust. Flame, vol. 163, pp. 394-405, 2016.

[82] S. Hemchandra and T. Lieuwen, "Local consumption speed of turbulent premixed flames - An analysis of "memory effects," Combust. Flame, vol. 157, no. 5, pp. 955-965, 2010.

[83] N. Popat et al., "Investigations to improve and assess the accuracy of computational fluid dynamic based explosion models," J. Hazard. Mater., vol. 45, no. 1, pp. 1-25, 1996.

[84] C. Catlin and R. Lindstedt, "Premixed turbulent burning velocities derived from mixing controlled reaction models with cold front quenching," Combust. Flame, vol. 85, no. 3-4, pp. 427-439, 1991.

[85] B. H. Hjertager, T. Solberg, and K. O. Nymoen, "Computer modelling of gas explosion propagation in offshore modules," J. Loss Prev. Process Ind., vol. 5, no. 3, pp. 165-174, 
1992.

[86] D. Bjerketvedt, J. R. Bakke, and K. Van Wingerden, "Gas explosion handbook," J. Hazard. Mater., vol. 52, no. 1, pp. 1-150, 1997.

[87] A. Van Den Berg, "REAGAS -A code for numerical simulation of 2-D reactive gas dynamics in gas explosion," 1989.

[88] D. Martin, "Some calculations using the two-dimensional turbulent combustion code FLARE," UK Atomic Energy Authority, 1986.

[89] T. Skjold, B. Arntzen, O. Hansen, O. Taraldset, I. Storvik, and R. Eckhoff, "Simulating dust explosions with the first version of DESC," Process Saf. Environ. Prot., vol. 83, no. 2, pp. 151-160, 2005.

[90] R. Abdel-Gayed, D. Bradley, and M. Lawes, "Turbulent burning velocities: A general correlation in terms of straining rates," Proc. R. Soc. A Math. Phys. Eng. Sci., vol. 414, no. 1847, pp. 389-413, 1987.

[91] P. Clavin and F. Williams, "Theory of premixed-flame propagation in large-scale turbulence," J. Fluid Mech., vol. 90, no. 3, pp. 589-604, 1979.

[92] V. Yakhot, "Propagation velocity of premixed turbulent flames," Combust. Sci. Technol., vol. 60, no. 1-3, pp. 191-214, 1988.

[93] V. Bychkov, "Velocity of turbulent flamelets with realistic fuel expansion," Phys. Rev. Lett., vol. 84, no. 26 Pt 1, pp. 6122-6125, 2000.

[94] O. Travnikov, V. Bychkov, and M. Liberman, "Numerical studies of flames in wide tubes: Stability limits of curved stationary flames," Phys. Rev. E, vol. 61, no. 1, pp. 468-474, 2000 .

[95] D. Valiev, "Flame dynamics and deflegration-to-detonation transition," Doctoral Thesis, Royal Institute of Technology, Stockholm, Sweden, 2008.

[96] O. Travnikov, M. Liberman, and V. Bychkov, "Stability of a planar flame front in a compressible flow," Phys. Fluids, vol. 9, no. 12, pp. 3935-3937, 1997.

[97] D. Valiev, V. Bychkov, V. Akkerman, and L. E. Eriksson, "Different stages of flame acceleration from slow burning to Chapman-Jouguet deflagration," Phys. Rev. E - Stat. Nonlinear, Soft Matter Phys., vol. 80, no. 3, 2009. 


\section{CURRICULUM VITAE}

Name:

Permanent residence:

Marital status:

Current occupation:

\section{Sinan Demir}

USA

Married

Graduate Research Assistant, PhD Candidate

Affiliations: Center for Alternative Fuels, Engines and Emissions (CAFEE) Center for Innovation in Gas Research and Utilization (CIGRU) Computational Fluid Dynamics and Applied Multi-Physics Center (CFD\&AMP) Department of Mechanical and Aerospace Engineering (MAE) West Virginia University (WVU), Morgantown, West Virginia, USA.

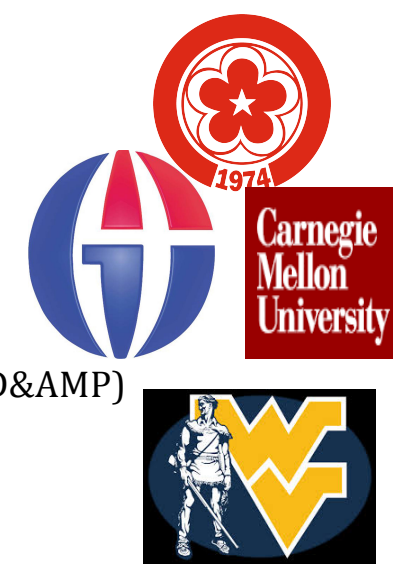

Mobile: $\quad+1-304-906-9061$

E-mail: $\quad$ ssinandemr@gmail.com; sidemir@mix.wvu.edu

\section{Professional and Academic Research Interests:}

\section{Theoretical and computational studies of}

- Morphology and dynamics of flame acceleration and deflagration-to-detonation transition (DDT) in gaseous and dusty-gaseous environments

- Combustion and hydrodynamic instabilities

- Fire and mining safety research

\section{Thermal design and optimization of}

- Energy \& power systems through energy, exergy, thermoeconomic and environmental analyses

\section{Education:}

Ph.D.

West Virginia University, Morgantown-WV-USA (08/2014-09/2017)

Department of Mechanical and Aerospace Engineering (GPA: 4.0)

Dissertation: Analytical and Computational Studies of Gaseous and Dusty

Combustion: From the Impacts of Local Flame Speed Variations to a Global

Predictive Mining Fire Scenario

Sponsors: Alpha Foundation, WVU, National Science Foundation (NSF).

Advisor: Dr. V'yacheslav (Slava) Akkerman

M.Sc. Gaziantep University, Gaziantep, TURKEY (2010-2012)

Department of Mechanical Engineering, Energy Division

Dissertation: Thermodynamic and Thermoeconomic Analyses and Optimization of Biogas Usage in Electricity and Hydrogen Productions from Wastewater Treatment Systems - Advisor: Dr. Aysegul Abusoglu

Sponsors: The Scientific and Technological Research Council of Turkey (TUBITAK)

B.Sc. $\quad$ Cumhuriyet University, Sivas, TURKEY (2006-2010)

Department of Mechanical Engineering, Energy Division 
Final Project: Performance Analysis of Different Airfoils for Wind Turbine Blades at Low Angles of Attack - Advisor: Dr. Melih Guleren

Work Experience:

Graduate Research Assistant (2014-Present)

West Virginia University, Morgantown-WV-USA

Graduate Teaching Assistant (2016)

West Virginia University, Morgantown-WV-USA

Internship (2014)

Carnegie Mellon University (CMU), Pittsburgh- PA-USA

Research Assistant (2010-2013)

Gaziantep University, Turkey

Mechanical Engineering Undergraduate Internship (2008-2009)

Management and Plant Organization + Design and Construction

Yesilyurt Iron and Steel Plant, Turkey

\section{Teaching Experience:}

West Virginia University, Morgantown, WV, USA (2016)

1. MAE 320 Thermodynamics

2. MAE 420 Internal Combustion Engines

Gaziantep University, Gaziantep, TURKEY (2011-2012)

1. ME 434 Exergy Method for Energy Systems

2. ME 568 Exergy Analysis and Thermoeconomics

Taught the engineering equation Solver (ESS) program to undergraduate and graduate students

Projects:

Promotion and Prevention of Flame Acceleration and Deflagration-to-Detonation Transition: from Fundamental Study to Practical Appl.- sponsored by National Science Foundation (May 2016- Present)

- Determined the influence of local variations of the thermal-chemical flame properties by means of analytical and computational studies adapted for parallel computations.

- Identified properties of a newly found near-sonic, quasi-steady deflagration.

- Investigated effect of thermal-diffusion instabilities, wall heat losses and fuel thermal expansion coefficient by means of computational simulations.

Development of a Gas and Dust Explosion Model- sponsored by Alpha Foundation (Aug. 2014- Aug. 2015)

- Derived predictive and quantitative analytical formulations of a mining accident, aiming to provide guidance for preventing and mitigating disasters associated with dusty-gaseous deflagrations and detonations in coal mines. 
- Developed a computational platform to reveal the mining fire hazards; the probability of spontaneous ignition; the evolution of a flame front; the likelihood of a deflagration-todetonation transition (DDT).

Thermodynamic and Thermoeconomic Analysis, Comparison and Optimization of Direct and Indirect Biogas Usage in Electricity and Hydrogen Productions from Waste Water Treatment Systemssponsored by The Scientific and Technological Research Council of Turkey (TUBITAK) (Nov 2010-Nov 2012)

- Detailed system and component analysis and optimization of a wastewater treatment plant and cogeneration facility by means of energy, economic, environmental analyses.

- Developed hydrogen production and storage models from biogas and waste energy.

- Assisted other various projects that include geothermal power plants.

\section{Performance Analysis of Different Airfoils for Wind Turbine Blades at Low Angles of Attack (Nov 2009-May 2010)}

- Creating computational scheme by using Gambit and analyzing aerodynamic performance of different wind turbine blades by using Fluent software.

\section{Awards/Scholarships/Fellowships:}

Name: $\quad$ Distinguished Doctoral Scholarship- Selected for a WVU Foundation Distinguished Doctoral Scholarship for the spring 2017 term. Award is in the amount of $\$ 5,000$.

Organization and Date: $\quad$ WVU- Office of Graduate Education \& Life, Morgantown, WV, USA. December 19, 2016.

Name: $\quad$ Presentation Award- Best Research Oral Presentation Awards out of 48 oral presenters.

Organization and Date: $\quad$ 92 $\quad$ WVAS- Glenville State College, WV, USA. April 8, 2017.

Name: $\quad$ Travel Fellowship - Sixteenth International Conference on Numerical Combustion (NC17) travel award. SIAM sponsored for $\$ 650$ travel award.

Organization and Date: NC17- Rosen Plaza Hotel in Orlando, Florida, USA. April 3-5, 2017.

Name: $\quad$ Travel Fellowship -KAUST Clean Combustion Research Center covers all the expenses for the Research Conference: New Combustion Concepts.

Organization and Date: KAUST, Thuwal, Saudi Arabia. March 6-8, 2017.

Name: $\quad$ Presentation Award- First place in excellence in oral presentation by a graduate student out of 47 oral presenters. WVAS sponsored for $\$ 150$.

Organization and Date: 91 st WVAS- Marshall University, WV, USA. April 9, 2016. 
Name: $\quad$ Poster Award- $2^{\text {nd }}$ MAE Research Fair - WVU sponsored awards worth $\$ 300$ for the best posters.

Organization and Date:

Name:

Organization and Date:

Name:

Organization and Date:

Name:

Organization and Date:

Name:

Organization and Date:

Name:

Organization and Date:

Name:

Organization and Date:
WVU- MAE Department, WV, USA. November 20, 2015.

Travel Award(s)- Combustion Institute sponsored travel awards for student participants in national combustion meetings.

$9^{\text {th }}$ U.S. National Combustion Meeting (May 17-20, 2015)- ESSCI Spring Meeting (March 13-16, 2016).

Poster Award-The Shared Research Facilities and the National Research Center for Coal and Energy sponsored travel awards worth $\$ 250$ each for the top three posters.

WVU High Performance Computing Day (HPC), WV, USA. April 16,2015.

Awarded Doctoral Scholarship (given to only 20 students in Turkey) TUBITAK (2014) (unable to accept due to conditions of scholarship).

Distinguished Master Thesis Award (2012-2013)- Thesis was ranked second out of the three that were given top thesis awards total out of approximately 80 theses.

Gaziantep University, 06/27/2013

Master Fellowship

TUBITAK (2010-2012)

Publication Incentive Award

TUBITAK, 2012

\section{Computer and Programming Skills:}

\section{$\square$ Fortran 77 \\ $\square$ C Programing \\ $\square$ Cantera}

$\square$ Engineering

Equation Solver

(EES) $\square$ Fortran 90/95

$\square$ Python

$\square$ Mac, Windows and Linux-

Ubuntu

$\square$ AutoCAD

\section{Publications:}

Book/Book Chapters: 1

Articles in refereed journals: 9

Articles under review and/or in submission process: $\mathbf{4}$

Conference contributions: $\mathbf{3 5}$

Invited talk/lectures: 3

Theses: 2 


\section{Book /Book Chapters:}

1. Abusoglu A., Demir S., Kanoglu M., Causes, Impacts, and Solutions to Global Warming, The Assessment of Sewage Sludge Potential from Municipal Wastewater Treatment Plants for Sustainable Biogas and Hydrogen Productions in Turkey, Springer, 2013. ISBN 978-1-4614-7587-3. http://www.springer.com/us/book/9781461475873

\section{Articles in Refereed Journals:}

9. Demir S., Calavay A.R., Akkerman V., Influence of Gas Compressibility on the Burning Accident in a Mining Passage-Combustion Theory and Modelling, 2017-Accepted

8. Demir S., Bychkov V., Chalagalla S.H.R., Akkerman V., Towards Predictive Scenario of a Burning Accident in a Mining Passage, Combustion Theory and Modelling, 2017Published online: http://dx.doi.org/10.1080/13647830.2017.1328129

7. Abusoglu A., Ozahi E., Kutlar, Demir S., I. Exergy analysis of green hydrogen production methods from biogas-based electricity and sewage sludge, International Journal of Hydrogen Energy, 42(16): 10986-10996, 2017.

6. Abusoglu A., Demir S., Ozahi E., Energy and economic analyses of models developed for sustainable hydrogen production from biogas-based electricity and sewage sludge, International Journal of Hydrogen Energy, 41(31): 13426-13435, 2016.

5. Abusoglu A., Demir S., Kanoglu M., Thermodynamic Analysis and Assessment of a Municipal Wastewater Treatment System: A Field Study on Sewage Treatment, International Journal of Exergy, 13(3): 281-298, 2013.

4. Abusoglu A., Demir S., Kanoglu M., Thermoeconomic Analysis of a Biogas Engine Powered Cogeneration System, Thermal Science and Technology, 33(2): 09-21 (in Turkish), 2013.

3. Abusoglu A., Demir S., Kanoglu M., Thermoeconomic Assessment of a Sustainable Municipal Wastewater Treatment System, Renewable Energy, 48: 424-35, 2012.

2. Abusoglu A., Demir S., Kanoglu M., Exergoeconomic Assessment of a Municipal Primary and Secondary Sewage Treatment, International Journal of Exergy, 11(3): 387405, 2012.

1. Guleren K.M., Demir S., Performance Analysis of Different Airfoils For Wind Turbine Blades at Low Angles of Attack, Thermal Science and Technology, 31(2): 51-59 (in Turkish), 2011. 


\section{Articles under Review and/or in Submission Process:}

4. Akkerman V., Demirgok B., Demir S., Bychkov V., Valiev D., Law C.K., Wu M.H., Acceleration of Ethylene-Oxygen Flames in Micro-Pipes: Theory, Experiment and Modelling, CNF-D-15-00498-under review

3. Demir S., Sezer H., Akkerman V., A Role of Local Planar Flame Speed Variations in the Global Finger-Flame Acceleration Scenario- TCTM-2017-07-76- under review

2. Demir S., Sezer H., Akkerman V., A Computational Study of Premixed Flame Propagation in Dusty-Gaseous Environment with Various Dust Distributions, submitted

1. Bilgili S., Demir S., Bychkov V., Morella H., Valiev D., Akkerman V., Impact of the Lewis and Markstein Numbers Effects on Flame Acceleration in Channels-submitted

\section{Conference Proceeding:}

35. 26 ${ }^{\text {th }}$ International Colloquium on the Dynamics of Explosions and Reactive Systems (ICDERS), Boston, USA. July 30-August 4, 2017

Demir S., Sezer H., Bush T., Akkerman V., Promotion and Mitigation of Premixed Flame Acceleration in Dusty-Gaseous Environment with Various Combustible Dust Distributions: A Computational Study. [Paper+Oral].

34. 10 ${ }^{\text {th }}$ U.S. National Combustion Meeting, University of Maryland, USA. April 23-26, 2017 Demir S., Bush T., Sezer H., Akkerman V., Computational Study of Premixed Flame Propagation in Dusty-Gaseous Environment with Various Dust Distributions. [Paper+Oral].

33. $10^{\text {th }}$ U.S. National Combustion Meeting, University of Maryland, USA. April 23-26, 2017 Shetty S.R., Demir S., Valiev D., Akkerman V., Impact of Thermal Expansion and Lewis Number on

Premixed Flame Propagation in Channels with Adiabatic and Isothermal, Nonslip Walls. [Paper+Oral].

32. 10 ${ }^{\text {th }}$ U.S. National Combustion Meeting, University of Maryland, USA. April 23-26, 2017 Demir S., Akkerman V., Work-in-Progress towards an Analytical Predictive Scenario of a Fire in a Coal mine. [Poster].

31. West Virginia Academy of Science, WV, USA. April 8, 2017 Demir S., Sezer H., Bush T., Akkerman V., Effect of Non-Uniform Coal Dust Distribution in a Gaseous-Dusty Premixed Environment on Flame Acceleration due to Wall Friction. [Oral].

30. West Virginia Academy of Science, WV, USA. April 8, 2017

Demir S., Akkerman V., Recent Progress towards a Predictive Scenario of a Methane-Air Coal Dust Fire in a Mining Passage. [Oral].

29. West Virginia Academy of Science, WV, USA. April 8, 2017 
Shetty S.R., Demir S., Akkerman V., Dynamics and Morphology of Premixed Flame Propagation in Channels: Effects of the Lewis Number and Thermal Boundary Conditions. [Oral].

28. West Virginia Academy of Science, WV, USA. April 8, 2017

Cathreno A., Adebiyi A., Demir S., Akkerman V., Premixed Flame Pulsations in Obstructed Channels with both Ends Open. [Poster].

27. 16 $^{\text {th }}$ International Conference on Numerical Combustion, Orlando, Florida, USA. April 3-5, 2017

Demir S., Sezer H., Rangwalla A., Akkerman V., Analytical and Computational Description of a Coal mine Fire Scenario. [Oral].

26. KAUST Research Conference: New Combustion Concepts, Thuwal, SA. March 6-8, 2017 Demir S., Akkerman V., Theory and Modeling of a Coal mine Fire Scenario. [Poster].

25. 42 ${ }^{\text {nd }}$ Dayton-Cincinnati Aerospace Sciences Symposium, USA. March 1, 2017

Demir S., Akkerman V., Effect of Gas Compression on Propane-Air Flames in Industrial Passages. [Oral].

24. 42 ${ }^{\text {nd }}$ Dayton-Cincinnati Aerospace Sciences Symposium, USA. March 1, 2017

Bush T., Demir S., Sezer H., Akkerman V., Modelling of Flame Acceleration due to Wall Friction in a Dusty-Gaseous Environment with Various Dust Distributions. [Oral].

23. 42 ${ }^{\text {nd }}$ Dayton-Cincinnati Aerospace Sciences Symposium, USA. March 1, 2017

Shetty S.R., Demir S., Valiev D., Akkerman V., Modelling of Flame Propagation in Channels with Nonslip Walls: The Impacts of the Lewis Number and the Thermal Expansion Coefficient. [Oral].

22. West Virginia Academy of Science, WV, USA. April 9, 2016

Demir S., Akkerman V., Rangwalla A., Predictive Scenario for Methane-Air-Dust Fires in a Mining Passage: Effect of Compressibility. [Oral].

21. West Virginia Academy of Science, WV, USA. April 9, 2016

Demir S., Sezer H., Akkerman V., Theoretical and Computational Studies of Wall Friction Flame Acceleration Mechanism with Linear Variation of Planar Flame Speed. [Oral].

20. 2016 ESSCI Spring Meeting, Princeton, NJ, USA. March 13-16, 2016.

Demir S., Sezer H., Akkerman V., A Role of Local Planar Flame Speed Variations in the Global Finger-Flame Acceleration Scenario. [Paper+Oral].

19. 2016 ESSCI Spring Meeting, Princeton, NJ, USA. March 13-16, 2016.

Demir S., Chalagalla S.H., Calavay A.R, Rangwala A.S, Akkerman V., Methane-Air-Dust Fire in a Mining Passage: The Role of Gas Compression in a Flame Acceleration Scenario.

[Paper+Oral].

18. 2016 ESSCI Spring Meeting, Princeton, NJ, USA. March 13-16, 2016.

Akkerman V., Bilgili S., Bychkov V., Valiev D., Demir S., Morella H., Impacts of the Lewis and 
Markstein numbers effects on the flame acceleration in channels. [Paper+Oral].

17. International Pittsburgh Coal Conference, Pittsburg, Pennsylvania, USA. October 5-8, 2015. Demir S., Sezer H., Akkerman V., Rangwala A.S., Theory and Modeling of Explosion Scenarios in Coal Mines. [Oral].

16. $9^{\text {th }}$ U.S. National Combustion Meeting, Cincinnati, Ohio, USA. May 17-20, 2015

Demir S., Sezer H., Akkerman V., Effect of Planar Flame Speed Variations on the Flame Acceleration Mechanisms. [Paper+Oral].

15. $9^{\text {th }}$ U.S. National Combustion Meeting, Cincinnati, Ohio, USA. May 17-20, 2015

Demir S., Sezer H., Akkerman V., Demirgok B., Rangwala A.S., Towards Modeling of DustyGaseous Fires in Coal Mines. [Paper+Oral].

14. $9^{\text {th }}$ U.S. National Combustion Meeting, Cincinnati, Ohio, USA. May 17-20, 2015

Akkerman V., Chalagalla S.H.R., Demir S., Rangwala A.S., Bychkov V., Predictive Scenario for Premixed Methane-Air Flame Spreading and Explosion Triggering in a Mining Passage. [Paper+Oral].

13. ASME Power \& Energy, San Diego, CA, USA-June 28-July 2, 2015

Demir S., Akkerman A., Rangwalla A., Bychkov V., Predictive Scenario for Methane-Air Fires in Coal Mines Associated with Gas and Dust Explosion. [Paper+Oral].

12. ASME Power \& Energy, San Diego, CA, USA-June 28-July 2, 2015

Ugarte U., Demir S., Demirgok B., Valiev D., Bychkov V., Akkerman A., Effect of Wall Heat Losses and Blockage Ratio on Flame Propagation in Obstructed Micro-Chambers. [Paper+Oral].

11. ASME Power \& Energy, San Diego, CA, USA-June 28-July 2, 2015

Demir S., Karabasoglu O., Akkerman A., Abusoglu A., Economic Optimization of Indirect Sewage Sludge Heat Dryer Unit for Sewage Sludge Incineration Plants. [Paper+Oral].

10. WVU High Performance Computing Day, HPC, WV, USA. April 16, 2015

Demir S., Sezer H., Demirgok B., Akkerman V., Rangwalla A., Bychkov V., Developing A Computational Platform for Mining Fire Safety. [Poster].

9. West Virginia Academy of Science, WV, USA. April 11, 2015

Demir S., Sezer H., Akkerman V., Rangwalla A., Bychkov V., Theory and Modeling of Flame Acceleration Mechanisms for Spatial Variations of Planar Flame Speed. [Oral].

8. West Virginia Academy of Science, WV, USA. April 11, 2015

Chalagalla S.H.R., Demir S., Akkerman V., Rangwalla A., Bychkov V., Predictive Scenario for Fires in Gas and Dust Environments: Premixed Flame Evolution. [Oral].

7. 40 $^{\text {th }}$ Dayton-Cincinnati Aerospace Sciences Symposium, USA. 4 March, 2015

Demir S., Akkerman A., Rangwalla A., Bychkov V., Predictive Scenario for Fires in Gaseous and Dust/Gas Environments: Premixed Flame Evaluation. [Oral].

6. $5^{\text {th }}$ International Conference on Development of EEE, Florence, Italy. November 22-24, 2014 
Abusoglu A., Demir S., Tozlu A., Energy and Economic Analyses of Models Developed for Sustainable Hydrogen Production from Biogas-Based Electricity and Sewage Sludge. [Paper+Oral].

5. Global Conference on Global Warming, GCGW - Istanbul, Turkey -July 8-12, 2012

Yilmaz C., Demir S., Abusoglu A., A Comparison of Exergoeconomic Methodologies for a Binary Geothermal Power Plant. [Paper+Oral].

4. Global Conference on Global Warming, GCGW - Istanbul, Turkey -July 8-12, 2012 Abusoglu A., Demir S., Kanoglu M., The Assessment of Sewage Sludge Potential from Municipal Wastewater Treatment Plants for Sustainable Biogas and Hydrogen Productions in Turkey. [Paper+Oral].

3. 6 $^{\text {th }}$ Int. Ege Energy Symposium and Exhibition -IEESE'6, Izmir, Turkey, June 28-30, 2012. Demir S., Abusoglu A., Kanoglu M., Thermoeconomic Optimization of a Wastewater Treatment Plant. [Paper+Oral].

2. $2^{\text {nd }}$ Int. Exergy, LCA and Sustain. Symposium, ELCAS'2 - Nsyros, Greece, June 19-21, 2011 Abusoglu A., Demir S., Kanoglu M., Thermodynamic Analysis and Assessment of Wastewater Treatment Plants in Scope of Anaerobic Sludge Digestion and On-Site Electricity Production from Biogas. [Paper+Oral].

1. Nat. Thermal Sci. and Technic Conf., ULIBTK'11- Zonguldak, Turkey, Sept.7-10, 2011

Demir S., Abusoglu A., Hepbasli A., Life Cycle Assessment of Electricity and Wastewater Sludge Produced by Biogas in Wastewater Treatment Plants. [Paper+Oral].

\section{Invited Talk/Lectures:}

3. West Virginia University-MEA Department, WV, USA. January 9, 2016

Fundamentals of Combustion

2. West Virginia University-MEA Department, WV, USA. November 28, 2016

Thermoeconomics-From the basics to a practical perspective

1. West Virginia University Institute of Technology, WV, USA. August 10-12, 2016

Combustion Summer Program for High-School Teachers

Language Skills:

English (proficient) and Turkish (native)

Membership- Organizations:

ASME, American Society of Mechanical Engineers, 2014-Present

CI, Combustion Institute- USA, 2014-Present

SIAM, Society for Industrial and Applied Mathematics, 2017-Present 


\section{References}

West Virginia University

Worcester Polytechnic Institute

West Virginia University

Carnegie Mellon University

Gaziantep University
Prof. Vyacheslav Akkerman

Vyacheslav.Akkerman@mail.wvu.edu

Prof. Ali S. Rangwala

rangwala@wpi.edu

Prof. Cosmin Dumitrescu

cedumitrescu@mail.wvu.edu

Prof. Orkun Karabasoglu

karabasoglu@cmu.edu

Prof. Mehmet Kanoglu

kanoglu@gantep.edu.tr 